pi-qg-213

ICMPA/MPA/04/2011

\title{
Ward-Takahashi identities for the colored Boulatov model
}

\author{
Joseph Ben Geloun ${ }^{*}$ \\ Perimeter Institute for Theoretical Physics, 31 Caroline St, Waterloo, ON N2L 2Y5, Canada \\ International Chair in Mathematical Physics and Applications, \\ ICMPA-UNESCO Chair, 072BP50, Cotonou, Rep. of Benin
}

\begin{abstract}
Ward-Takahashi identities of the colored Boulatov model are derived using a generic unitary field transformation. In a specific instance, this generic transformation turns out to be a symmetry of the interaction so that particular classes of reduced Ward-Takahashi identities for that symmetry are consequently identified and interpreted.
\end{abstract}

Pacs numbers: 04.60.Gw, 05.10.Cc, 11.10.Gh

Key words: Group field theory, Ward-Takahashi identities

\section{INTRODUCTION}

Group field theories (GFTs) are generalization of matrix models as higher rank tensor quantum field theories over a group manifold [1, 2]. They provide a relevant framework for the quantization of gravity [3 -7$]$ as well as possess, as recently shown for the particular class of colored GFT models [8 10, an equivalent formulation of a large $1 / N$ limit expansion [11 13] and exhibit a critical behavior [14, 15].

It should be emphasized that the presence of colored fields in GFT plays an increasing role in the search of symmetry of these theories. Indeed, some investigations pertaining to the symmetry aspects of such colored GFTs have been led recently [16-20] and one proved that, to mention a few, the colored theories are endowed with a genuine quantum group symmetry [16] which encodes a notion of diffeomorphism symmetry in GFTs [18] and by direct computations in group space, they prove to possess a conserved quantity associated with translation symmetry [20]. All these properties have been only proved for the colored case and there is, at the moment, no hint of how they could be implemented in the non colored situation.

Let us remind that the partition functions of GFT models in the sense of Boulatov-Ooguri [1, 2] are defined through a nonlocal interaction and a Gaussian measure with a degenerate covariance. This covariance is indeed made of a group averaging and product of delta functions which in fact projects onto the gauge invariant sector of the space of square integrable functions $L^{2}\left(G^{D}, \mathbb{C}\right)$, for a $D$ dimensional GFT. Hence the kinetic term in the Lagrange formulation of the action, can be seen as trivial (this term is of the mass kind when restricting field on the gauge invariant sector) or even inexistent from the pure quantum field theory point of view. Besides, it is well-known that the ordinary notion of symmetry in a presence of nonlocal interaction becomes more involved or even could break down. These peculiar features, in returns, render unclear the ordinary definition of classical symmetry and the corresponding notion of Noether theorem (the notion of Noether currents in a trivial dynamical theory may only reduce to the Lagrangian density itself for translations for instance). At the current stage of investigations on symmetries of GFTs, there are three ways to address these issues: either to deal with a quantum group symmetry and making use of Hopf algebra techniques [16], or to introduce a nontrivial kinetic term [20] (motivated indeed by renormalization requirements in 21] [5]), or, finally, to state directly the Noether theorem for a given symmetry at the quantum level. The latter is well-known to be related with the identification of Ward-Takahashi (WT) identities associated with a particular field symmetry. To shed more light on the last aspect is the purpose of the present work.

A review some basic but important facts of WT identities in general quantum field theory and precisions on what can be investigated in our situation is in order. WT identities are consequences of a symmetry of a system at the quantum level (for instance in path integral formalism) in the form of relations between correlation functions. These identities should hold even after renormalization. As an illustration, in quantum electrodynamics with its $U(1)$ gauge symmetry, the WT identities relate the full three-point function with the two-point function hence the wave function renormalization with the vertex renormalization. It is nevertheless true that, in an all-embracing view, a given field transformation without requiring any action symmetry induces relations between correlation functions that can be

* jbengeloun@perimeterinstitute.ca 
legitimately called WT identities. A known example of these occurs in the context of noncommutative quantum field theory (also a nonlocal theory) mapped onto non identically distributed matrix models [29. The dynamics explicitly breaks the ordinary $U(N)$ symmetry of matrix models and, at the quantum level, produces WT identities. These latter play a crucial role in the proof of asymptotic safety at all orders of perturbation theory [30, 31. Hence, having or not a symmetry of an action, some relations between correlation functions can be unraveled provided one has a well defined field transformation. These relations yield a weaker sense of equations of motion which in return can be related to modified (quantum) conservation law for currents associated with that transformation. WT identities therefore encode the obstructions of a theory to be invariant at the quantum level, obstructions which are useful in the renormalization program and also from the point of view of generalizing the sense of symmetry of a given system. Let us come back to our situation dealing with a strict quantum tensor field theory without classical dynamics and possessing a nonlocal interaction. It becomes obvious that for reasons invoked so far (the general concept of symmetry and its influence on the renormalization program) investigating WT identities is certainly significant in the GFT understanding. More specifically, one may ask: can we make a (whatsoever general) sense of symmetries with conserved currents by stating them directly at the quantum level using WT identities ? This work will give a partial but positive answer to that question provided one uses colored fields.

In this paper, we define a generic unitary field transformation from which are studied WT identities of the colored Boulatov model [1]. This general unitary field transformation turns out to be a symmetry for the Boulatov action provided a specific way that one chooses to act on the field arguments. Associated with that symmetry, reduced WT equations satisfied by the correlations functions are determined and analyzed. We find that these reduced WT identities can be interpreted as quantum equation of motions for an underlying classical theory with a trivial kinetic term. Hence, bearing in mind that the field equation of motion is closely related to the conservation law of any kind of symmetry, it is not hopeless that WT relations obtained in this nonlocal theory could describe the same idea of modified conservation law stated at the quantum level once one makes a sense of classical conserved charges (this question will be not treated here). Moreover, the various kind of identities carried out should be useful for in-depth perturbative and nonperturbative renormalization programs of GFTs [19, 21 28. Finally, the results achieved can be extended in any GFT dimension.

The paper is organized as follows: Section 2 reviews the basics of the Boulatov model and discusses the main strategy to obtain WT identities from a field symmetry and the universal feature that they will assume for any tensor models. Section 3 introduces the unitary field transformation that will define the variations of the different parts entering in the definition of the partition function. Mainly, one can define a general unitary transformation and one more specific unitary with the property that it preserves the gauge invariance of fields and is a symmetry of the interaction term. For the general and the more specific symmetry the study of WT identities are discussed in Section 4. A summary and outlook of the results are given in Section 5 and, finally, a detailed appendix provides basic facts on the theory, on unitary field transformations and other precisions on derivations used in the text.

\section{THE COLORED BOULATOV MODEL}

Let $G$ be some compact multiplicative Lie group, denote $e$ its unit, and $\int d h=: \int_{h}$ the integral with respect to the Haar measure. Consider $\bar{\varphi}^{i}, \varphi^{i}, i=0,1,2,3$ some complex scalar fields over three copies of $G, \varphi^{i}: G^{3} \rightarrow \mathbb{C}$. We denote $\delta^{N}(h)$ the delta function over $G$ that we cutoff such that $\delta^{N}(e)$ is finite, but diverges as $N$ goes to infinity. In the specific situation of $G=S U(2)$ which will be focus of this paper (respectively $G=U(1)$ ), one has

$$
\left.\delta^{N}(h)\right|_{G=S U(2)}=\sum_{j=0}^{N} d_{j} \chi^{j}(h) \quad\left(\text { resp. }\left.\quad \delta^{N}(\varphi)\right|_{G=U(1)}=\sum_{p=-N}^{N} e^{\imath p \varphi}\right),
$$

where $\chi^{j}(h)$ stands for the character of group element $h$ in the representation $j$ and $d_{j}=2 j+1$.

The following path integral defines the partition function of the colored Boulatov model [8] over $G$ :

$$
Z(\lambda, \bar{\lambda})=e^{-F(\lambda, \bar{\lambda})}=\int \prod_{i=0}^{4} d \mu_{C}(\bar{\varphi}, \varphi) e^{-S^{\mathrm{int}}\left(\bar{\varphi}^{i}, \varphi^{i}\right)},
$$

with normalized Gaussian measure $d \mu_{C}(\bar{\varphi}, \varphi)$ of covariance $C$ (denoting henceforth $\varphi_{h p q}:=\varphi(h, p, q)$ ):

$$
C_{h_{0} h_{1} h_{2} ; h_{0}^{\prime} h_{1}^{\prime} h_{2}^{\prime}}^{k j}=\int d \mu_{C}(\bar{\varphi}, \varphi) \bar{\varphi}_{h_{0} h_{1} h_{2}}^{k} \varphi_{h_{0}^{\prime} h_{1}^{\prime} h_{2}^{\prime}}^{j}=\delta^{k j} \int_{h} \delta^{N}\left(h_{0} h\left(h_{0}^{\prime}\right)^{-1}\right) \delta^{N}\left(h_{1} h\left(h_{1}^{\prime}\right)^{-1}\right) \delta^{N}\left(h_{2} h\left(h_{2}^{\prime}\right)^{-1}\right),
$$

and interaction wisely chosen

$$
S^{\mathrm{int}}=\frac{\lambda}{\sqrt{\delta^{N}(e)}} \int_{h_{i j}} \varphi_{h_{03} h_{02} h_{01}}^{0} \bar{\varphi}_{h_{10} h_{13} h_{12}}^{1} \varphi_{h_{21} h_{20} h_{23}}^{2} \bar{\varphi}_{h_{32} h_{31} h_{30}}^{3}
$$




$$
+\frac{\bar{\lambda}}{\sqrt{\delta^{N}(e)}} \int_{h_{i j}} \bar{\varphi}_{h^{03} h^{02} h^{01}} \varphi_{h^{10} h^{13} h^{12}}^{1} \bar{\varphi}_{h^{21} h^{20} h^{23}}^{2} \varphi_{h^{32} h^{31} h^{30}}^{3}
$$

where one should identify $h_{i j}=h_{j i}$ and the symbol $\int_{h_{i j}}$ means that the integral is performed over all variables labelled by $h_{i j}$ (here above six copies of $G$ ). The resulting pairing of field arguments of this nonlocal interaction can be dually and graphically associated with a tetrahedron (each argument for each edge) and Feynman graphs, in this theory, are noting but a collection of tetrahedra (simplicial complexes) glued along one of their faces (triangle) following the covariance rule. More precisions on the GFT diagrammatics can be found in [4. For a colored theory, there is an additional gluing constraint enforcing that only colors of the same index can be glued together (hence the presence of $\delta^{j k}$ in the covariance). One also notes that, in the ordinary colored GFT [8], the interaction with coupling constant $\lambda$ is of the form $\varphi^{0} \varphi^{1} \varphi^{2} \varphi^{3}$ and the one with coupling $\bar{\lambda}$ can be chosen as its complex conjugate. Here, we have just renamed $\varphi^{1,3}$ as $\bar{\varphi}^{1,3}$ (and vice-versa) in order to have a correct notion of field transformation with respect to colors. Hence, the formalism that we will develop holds without loss of generality in the ordinary colored GFT, with another field transformation.

As an operator, $C$ can act also onto functions. We will use indifferently the compact notations, when no confusion may occur

$$
\begin{aligned}
& {[C \varphi]_{[g]}^{i}=C_{[g][\tilde{g}]}^{i j} \varphi_{[\tilde{g}]}^{j}=\int_{[\tilde{g}]} C_{[g][\tilde{g}]}^{i j} \varphi_{[\tilde{g}]}^{j}:=\int_{\tilde{g}_{\alpha}} C_{g_{1} g_{2} g_{3} ; \tilde{g}_{1} \tilde{g}_{2} \tilde{g}_{3}}^{i j} \varphi_{\tilde{g}_{1} \tilde{g}_{2} \tilde{g}_{3}}^{j},}
\end{aligned}
$$

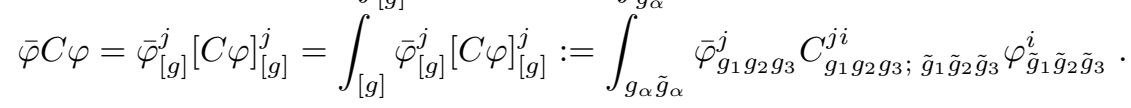

We sketch here the main steps of our program that will allow us to extract the WT identities. Foremost, we should stress that albeit the entire study is realized in three dimensions, the same analysis can be easily carried out for arbitrary dimension with not so much effort: for instance, any symbol $[g]$ will be just adjusted to be $D$ copies of group elements (note that most of the appendices discusses the $D$ dimensional situation). The only issue there will be to trace in the interaction the correct group element with respect to which the transformation is realized and then to adapt again our notations for that context. Furthermore, in the next paragraph, we discuss the method for getting WT identities and their main features which should be valid in full generality, for any kind of field transformation $\varphi \rightarrow \tilde{\varphi}$ (save the identity for which everything obviously trivializes), for any tensor model and in arbitrary dimension. We will emphasize the specificities of GFT models at each major step.

As initial data, consider a partition function $Z$ defined with a Gaussian measure $d \mu_{C}(\varphi)$ of covariance $C$ (which could be the identity, any invertible operator or even projector as it occurs in GFTs), an interaction $S^{\text {int }}$ and also source terms $J \varphi$. Second, consider a field transformation yielding at the infinitesimal level a nontrivial variation $\delta_{\epsilon} \varphi \neq 0$, with $\epsilon$ the infinitesimal parameter associated with that transformation. Collect the variations of the above quoted constituents of the partition function, namely $\delta_{\epsilon} S^{\text {int }}, \delta(J \varphi)=J \delta_{\epsilon} \varphi$ (assumed to be never vanishing) and last, more interestingly, $\delta_{\epsilon} d \mu_{C}(\varphi)$. Then, many cases can occur: $\delta_{\epsilon} S^{\text {int }}=0$ or not, and $\delta_{\epsilon} d \mu_{C}(\varphi)=0$ or not. Whenever $\delta_{\epsilon} S^{\text {int }}=0$ then the transformation is a symmetry of the interaction part. Meanwhile $\delta_{\epsilon} d \mu_{C}(\varphi)=0$ means that the transformation does not affect the kinetic term. As an example, considering any independent identically distributed (i.i.d.) tensor model with $C$ is the identity operator, an unitary symmetry will exactly produce a result of this kind whereas all GFT models endowed with a gauge projector as (3), the analogous part will have a nontrivial contribution. The next stage is to differentiate of the free energy $\ln Z$ (generating function of connected components) with respect to the infinitesimal parameter $\epsilon$ and to equate obviously the result to zero. If $\delta_{\epsilon} S^{\text {int }}$ or $\delta_{\epsilon} d \mu_{C}(\varphi)$ are non zero, then one expects to get a priori relevant contributions. Pursuing the analysis, one differentiates the equation with respect to source fields $J$ a given number of time and then puts these sources at zero. The result is a generalized WT identity which can be decomposed in two parts: a contribution coming from $J \delta_{\epsilon} \varphi$ and other contributions issued from $\delta_{\epsilon} S^{\text {int }}$ and $\delta_{\epsilon} d \mu_{C}(\varphi)$. In an ordinary local theory, these last mentioned contributions are nothing but expectation values of field equations of motion which can be related to a modified conservation property of the currents associated with the transformation. Thus, WT identities can be interpreted as a quantum version of the local conservation of these currents. In our tensor situation, the sense of conserved charges is less clear without true dynamics. But still, as we will show after some manipulations, the WT identities find a relation with equations of motion of an auxiliary GFT theory. Going back to the general setting, a final stage is to analyze and to reduce these WT identities and to interpret them graphically. We can comment that for i.i.d. models with an invariant interaction under some symmetry, WT identities only give relations between $N$-point functions (of the same species, i.e. with $N$ external legs) because the only contributions to the differentiation will come from the source terms $J \delta_{\epsilon} \varphi$. In GFT models implemented with an invariant interaction, due to the contribution of $\delta_{\epsilon} d \mu_{C}(\varphi)$, WT equations will definitely mix different kinds of $N$-point functions henceforth worthy to be scrutinized. Then, the bottom line is the definition of a field transformation and this is the purpose of the next section. 


\section{UNITARY TRANSFORMATIONS}

\section{A. General unitary transformations}

From now on, we will restrict to the situation where $G=S U(2)$ though most of the ensuing developments may found an extension for an arbitrary Lie group.

Consider the partition function $Z$ including source terms and the associated free energy $W$ for the Boulatov model, namely

$$
e^{-W(\bar{\eta}, \eta)}=Z(\bar{\eta}, \eta)=\int d \mu_{C}(\bar{\varphi}, \varphi) e^{-S^{\text {int }}(\varphi, \bar{\varphi})+\bar{\varphi} \eta+\varphi \bar{\eta}}
$$

where notations remain the same as earlier and the source term incorporates color indices:

$$
\bar{\varphi} \eta+\varphi \bar{\eta}:=\sum_{i} \int_{g_{\alpha}}\left(\bar{\varphi}_{g_{1} g_{2} g_{3}}^{i} \eta_{g_{1} g_{2} g_{3}}^{i}+\bar{\eta}_{g_{1} g_{2} g_{3}}^{i} \varphi_{g_{1} g_{2} g_{3}}^{i}\right) .
$$

In order to avoid confusion, we will use different notations for a single index $a$ and a triple index $[a]=\left(a_{1}, a_{2}, a_{3}\right)$. Hence the composition of the covariance and a field will be written henceforth $C_{[a][b]} \varphi_{[b]}$ and an operator $T$ acting onto a single group element of fields will be denoted as $T_{a b} \varphi_{b}$.

Let us consider now a general unitary operator $U$ satisfying a composition law $U_{[a][b]}^{i j} \bar{U}_{[c][b]}^{i^{\prime} j}=\delta^{i i^{\prime}} \delta_{[a][c]}$, with $\delta_{[a][c]}$ the kernel of the unit operator, such that the fields transform under $U$ as

$$
U \varphi_{[a]}^{i}=U_{[a][b]}^{i j} \varphi_{[b]}^{j}, \quad{ }^{U} \bar{\varphi}_{[a]}^{i}=\bar{\varphi}_{[b]}^{j} \bar{U}_{[a][b]}^{i j}, \quad \frac{\delta}{\delta^{U} \varphi_{[a]}^{i}}=\frac{\delta}{\delta \varphi_{[b]}^{j}}\left(U^{-1}\right)_{[b][a]}^{j i}, \quad \frac{\delta}{\delta^{U} \bar{\varphi}_{[a]}^{i}}=U_{[a][b]}^{i j} \frac{\delta}{\delta \bar{\varphi}_{[b]}^{j}}
$$

(one can prove that such operators exist, see Appendices B and B 2). Let us keep at the moment these as formal expressions.

Under $U, S^{\text {int }}$ transforms as

$$
\begin{aligned}
U S^{\mathrm{int}}= & \frac{\lambda}{\sqrt{\delta^{N}(e)}} \int_{h_{i j}} U \varphi_{h_{03} h_{02} h_{01}}^{0} \bar{\varphi}_{h_{10} h_{13} h_{12}} U \varphi_{h_{21} h_{20} h_{23}}^{2} \bar{\varphi}_{h_{32} h_{31} h_{30}}^{3} \\
& +\frac{\bar{\lambda}}{\sqrt{\delta^{N}(e)}} \int_{h_{i j}} \bar{\varphi}_{h^{03} h^{02} h^{01}}^{0} \varphi_{h^{10} h^{13} h^{12}}^{1} U_{h^{21} h^{20} h^{23}}^{U^{2}} \varphi_{h^{32} h^{31} h^{30}}^{3}, \\
= & \frac{\lambda}{\sqrt{\delta^{N}(e)}} \int_{h_{i j}} \int_{[a][b][c][d]} U_{h_{03} h_{02} h_{01}[a]}^{i_{0}} \varphi_{[a]}^{i_{0}} \bar{\varphi}_{[b]}^{i_{1}} \bar{U}_{h_{10} h_{13} h_{12}[b]}^{1 i_{1}} U_{h_{21} h_{20} h_{23}[c]}^{2 i_{2}} \varphi_{[c]}^{i_{2}} \bar{\varphi}_{[d]}^{i_{3}} \bar{U}_{h_{32} h_{31} h_{30}[d]}^{3 i_{3}} \\
& +\frac{\bar{\lambda}}{\sqrt{\delta^{N}(e)}} \int_{h_{i j}} \int_{[a][b][c][d]} \bar{\varphi}_{[a]}^{i_{0}} \bar{U}_{h^{03} h^{02} h^{01}[a]}^{0 i_{0}} \varphi_{[b]}^{i_{1}} U_{h^{10} h^{13} h^{12}[b]}^{1 i_{1}} \bar{\varphi}_{[c]}^{i_{2}} \bar{U}_{h^{21} h^{20} h^{23}[c]}^{2 i_{2}} U_{h^{32} h^{31} h^{30}[d]}^{3 i_{3}} \varphi_{[d]}^{i_{3}} .
\end{aligned}
$$

The partition function under (8) can be mapped onto (see Eq. (C.6) of Appendix C 1)

$$
Z(\bar{\eta}, \eta)=\int d \mu_{U C U^{-1}}(\bar{\varphi}, \varphi) e^{-S^{\mathrm{int}}\left(U \varphi, \bar{\varphi} U^{-1}\right)+\bar{\varphi} U^{-1} \eta+\bar{\eta} U \varphi} .
$$

By Lemma 3 (see Appendix A), we write this modified partition function as

$$
Z(\bar{\eta}, \eta)=\int d \mu_{C}(\bar{\varphi}, \varphi) e^{\delta_{\varphi}\left(U C U^{-1}-C\right) \delta_{\bar{\varphi}}} e^{-S^{\mathrm{int}}\left(U \varphi, \bar{\varphi} U^{-1}\right)+\bar{\varphi} U^{-1} \eta+\bar{\eta} U \varphi} .
$$

Close to the identity, we can set $U_{[a][b]}^{i j}=\delta^{i j} \delta_{[a][b]}+\imath B_{[a][b]}^{i j}$, where $B$ is a Hermitian kernel in the sense that $\bar{B}_{[b][a]}^{j i}=$ $B_{[a][b]}^{i j}$. At first order in $B$, the variation of the interaction part is of the form (see Appendix $\mathrm{C} 1$ for precisions concerning the notations and derivations of the following infinitesimal variations)

$$
\begin{aligned}
& \delta_{B} S^{\text {int }}:=\imath \lambda\left[[B \varphi]^{0} \bar{\varphi}^{1} \varphi^{2} \bar{\varphi}^{3}-\varphi^{0}[\bar{\varphi} B]^{1} \varphi^{2} \bar{\varphi}^{3}+\varphi^{0} \bar{\varphi}^{1}[B \varphi]^{2} \bar{\varphi}^{3}-\varphi^{0} \bar{\varphi}^{1} \varphi^{2}[\bar{\varphi} B]^{3}\right]+\imath \bar{\lambda}\{\bar{\varphi}\}, \\
& \bar{\lambda}\{\bar{\varphi}\}=-\left[\bar{\varphi}^{0} B\right] \varphi^{1} \bar{\varphi}^{2} \varphi^{3}+\bar{\varphi}^{0}[B \varphi]^{1} \bar{\varphi}^{2} \varphi^{3}-\bar{\varphi}^{0} \varphi^{1}[\bar{\varphi} B]^{2} \varphi^{3}+\bar{\varphi}^{0} \varphi^{1} \bar{\varphi}^{2}[B \varphi]^{3} .
\end{aligned}
$$

Meanwhile, the source terms have the infinitesimal transformations

$$
\delta_{B}(\bar{\eta} \varphi+\bar{\varphi} \eta)=\imath \sum_{i} \int_{[g][a]}\left(-\bar{\varphi}_{[a]}^{i} B_{[a][g]}^{i j} \eta_{[g]}^{j}+\bar{\eta}_{[g]}^{i} B_{[g][a]}^{i j} \varphi_{[a]}^{j}\right)=: \imath(-\bar{\varphi} B \eta+\bar{\eta} B \varphi)
$$

and varying the covariance, we get

$$
\left[U C U^{-1}\right]_{[a][b]}^{i j}-C_{[a][b]}^{i j}=\imath\left[B_{[c][a]}^{l i} C_{[c][b]}^{l j}-C_{[a]\left[c^{\prime}\right]}^{i l^{\prime}} B_{[b]\left[c^{\prime}\right]}^{j l^{\prime}}\right]=: \imath[B C-C B]_{[a][b]}^{i j} .
$$




\section{B. Right invariant unitary transformations}

Among unitary operators, there exists a particular class that we propose also to study. This class includes unitary operators called right invariant unitaries for having the particular property to preserve the gauge invariance of fields ${ }^{1}$ and let $S^{\text {int }}$ invariant. A detailed discussion about these right invariant unitaries can be found in Appendix B 1.

Working with a right invariant unitary $U$ in the sense of the 1-action of Appendix B 2 Eq. (B.19), we get a change of field variables such that

$$
\begin{aligned}
& { }^{U} \varphi_{a .}^{0}=U_{a b} \varphi_{b .}^{0}, \quad{ }^{U} \bar{\varphi}_{a .}^{0}=\bar{\varphi}_{b .}^{0}\left(U^{-1}\right)_{b a}=\bar{U}_{a b} \bar{\varphi}_{b .}^{0}, \quad \frac{\delta}{\delta \varphi_{a .}^{0}}=\frac{\delta}{\delta \varphi_{b .}^{0}}\left(U^{-1}\right)_{b a}, \quad \frac{\delta}{\delta \bar{\varphi}_{a .}^{0}}=U_{a b} \frac{\delta}{\delta \bar{\varphi}_{b .}^{0}}, \\
& U_{\varphi^{1} a}^{1}=U_{a b} \varphi_{. b}^{1}, \quad U_{\bar{\varphi}_{. a}^{1}}=\bar{\varphi}_{. b}^{1}\left(U^{-1}\right)_{b a}=\bar{U}_{a b} \bar{\varphi}_{. b}^{1}, \quad \frac{\delta}{\delta^{U} \varphi_{. a}^{1}}=\frac{\delta}{\delta \varphi_{. b}^{1}}\left(U^{-1}\right)_{b a}, \quad \frac{\delta}{\delta^{U} \bar{\varphi}_{. a}^{1}}=U_{a b} \frac{\delta}{\delta \bar{\varphi}_{. b}^{1}}
\end{aligned}
$$

and colors 2 and 3 transform like 0 and 1, respectively. The subscript $a$ or $b$ refers to a unique group element and the dot notifies the position of the remaining arguments. Thus 0 and 2 are transformed with respect to their first argument whereas 1 and 3 to their last argument.

$S^{\text {int }}$ remains invariant under this transformation (see Appendix C 2 for details of the identities in the remaining of this section)

$$
U S^{\text {int }}=S^{\text {int }}
$$

In fact, for the colored Boulatov model there are six such right invariant unitaries, namely one for each couple of arguments in the interaction, leaving the colored GFT interaction invariant. More generally, a $D$ dimensional colored GFT will be invariant under $D(D+1) / 2$ of such basic transformations that one can think as minimal symmetries. In the following, we will use one of these minimal symmetry in order to simplify some general WT identities and, consequently, to prove that the WT identities derived in this work have a nontrivial content.

Under (15), the partition function takes the form

$$
Z(\bar{\eta}, \eta)=\int d \mu_{U C U^{-1}}(\bar{\varphi}, \varphi) e^{-S^{\mathrm{int}}\left(U \varphi, \bar{\varphi} U^{-1}\right)+\bar{\varphi} U^{-1} \eta+\bar{\eta} U \varphi}
$$

where the action of $U$ on the covariance is defined by

$$
\begin{aligned}
& \bar{U}_{h_{0}^{\prime} c^{\prime}} C_{h_{0} h_{1} h_{2} ; h_{0}^{\prime} h_{1}^{\prime} h_{2}^{\prime}}^{i i=0, U_{h_{0} c}}=\int d \mu_{C}\left({ }^{U} \bar{\varphi},{ }^{U} \varphi\right) \int_{a b h_{0} h_{0}^{\prime}} U_{h_{0} c} \bar{U}_{h_{0} a} \bar{U}_{h_{0}^{\prime} c^{\prime}} U_{h_{0}^{\prime} b} \bar{\varphi}_{a h_{1} h_{2}}^{i=0,2} \varphi_{b h_{1}^{\prime} h_{2}^{\prime}}^{i=0,2}:=\left[U C U^{-1}\right]_{c h_{1} h_{2} ; c^{\prime} h_{1}^{\prime} h_{2}^{\prime}}^{i i=0,2} \\
& \bar{U}_{h_{2}^{\prime} c^{\prime}} C_{h_{0} h_{1} h_{2} ; h_{0}^{\prime} h_{1}^{\prime} h_{2}^{\prime}}^{i i=1, U_{h_{2} c}}=\int d \mu_{C}\left({ }^{U} \bar{\varphi},{ }^{U} \varphi\right) \int_{a b h_{2} h_{2}^{\prime}} U_{h_{2} c} \bar{U}_{h_{2} a} \bar{U}_{h_{2}^{\prime} c^{\prime}} U_{h_{2}^{\prime} b} \bar{\varphi}_{h_{0} h_{1} a}^{i=1,3} \varphi_{h_{0}^{\prime} h_{1}^{\prime} b}^{i=1,3}:=\left[U C U^{-1}\right]_{h_{0} h_{1} c ; h_{0}^{\prime} h_{1}^{\prime} c^{\prime}}^{i i=1,3} \cdot(18)
\end{aligned}
$$

Turning the discussion to infinitesimal transformations, we have $\delta_{B} S^{\text {int }}=0$,

$$
\begin{gathered}
{\left[U C U^{-1}\right]_{[a][b]}^{i i=0,2}-C_{[a][b]}^{i i=0,2}=\imath\left[-C_{[a] ; c^{\prime} b_{1} b_{2}}^{i i=0,2} B_{b_{0} c^{\prime}}+B_{c a_{0}} C_{c a_{1} a_{2} ;[b]}^{i i=0,2}\right]=: \imath[B C-C B]_{[a][b]}^{i i=0,2},} \\
{\left[U C U^{-1}\right]_{[a][b]}^{i i=1,3}-C_{[a][b]}^{i i=1,3}=\imath\left[-C_{[a] ; b_{0} b_{1} c^{\prime}}^{i i=1,3 b_{2} c^{\prime}}+B_{c a_{2}} C_{a_{0} a_{1} c ;[b]}^{i i=1,3}\right]=: \imath[B C-C B]_{[a][b]}^{i i=1,3},}
\end{gathered}
$$

whereas varying source terms yields

$$
\begin{aligned}
& \delta_{B}(\bar{\eta} \varphi+\bar{\varphi} \eta)=\sum_{i} \int_{[g]}\left(U^{U} \bar{\varphi}_{[g]}^{i} \eta_{[g]}^{i}+\bar{\eta}_{[g]}^{i} U_{\varphi_{[g]}}^{i}\right)-(\bar{\eta} \varphi+\bar{\varphi} \eta) \\
& =\imath \int_{g_{i} a}\left\{\sum_{i=0,2}\left(-\bar{\varphi}_{a .}^{i} B_{a g_{0}} \eta_{g_{0} .}^{i}+\bar{\eta}_{g_{0} .}^{i} B_{g_{0} a} \varphi_{a .}^{i}\right)+\sum_{i=1,3}\left(-\bar{\varphi}_{. a}^{i} B_{a g_{2}} \eta_{. g_{2}}^{i}+\bar{\eta}_{. g_{2}}^{i} B_{g_{2} a} \varphi_{. a}^{i}\right)\right\} \\
& =: \imath(-\bar{\varphi} B \eta+\bar{\eta} B \varphi) .
\end{aligned}
$$

Having collected all infinitesimal terms, we are in position to study the WT identities of the model and that will be the focus of the rest of this work.

\footnotetext{
1 GFTs can be indeed defined with gauge invariant fields. Hence, the mentioned transformation will preserve this property of fields.
} 


\section{WARD-TAKAHASHI IDENTITIES FOR THE COLORED BOULATOV MODEL}

\section{A. General unitary transformation}

Our starting point is the partition function (11). Considering the infinitesimal transformations $(12),(13)$ and $(14)$, $Z$ may be written

$$
\begin{aligned}
& Z(\bar{\eta}, \eta)=\int d \mu_{C}(\bar{\varphi}, \varphi)\left(1+\imath \delta_{\varphi}[B C-C B] \delta_{\bar{\varphi}}\right) e^{-S^{\mathrm{int}}(\varphi, \bar{\varphi})-\delta_{B} S^{\mathrm{int}}+\bar{\varphi} \eta+\bar{\eta} \varphi+\delta_{B}(\bar{\varphi} \eta+\bar{\eta} \varphi)} \\
& =\int d \mu_{C}(\bar{\varphi}, \varphi)\left(1+\imath \delta_{\varphi}[B C-C B] \delta_{\bar{\varphi}}\right) e^{-S^{\mathrm{int}}(\varphi, \bar{\varphi})+\bar{\varphi} \eta+\bar{\eta} \varphi \times} \\
& \left\{1-\imath \bar{\varphi} B \eta+\bar{\eta} B \varphi-\imath \lambda\left[[B \varphi]^{0} \bar{\varphi}^{1} \varphi^{2} \bar{\varphi}^{3}-\varphi^{0}[\bar{\varphi} B]^{1} \varphi^{2} \bar{\varphi}^{3}+\varphi^{0} \bar{\varphi}^{1}[B \varphi]^{2} \bar{\varphi}^{3}-\varphi^{0} \bar{\varphi}^{-1} \varphi^{2}[\bar{\varphi} B]^{3}\right]-\imath \bar{\lambda}\{\bar{\varphi}\}\right\} .
\end{aligned}
$$

Being interesting only on connected functions, we now derivate the free energy with respect to the infinitesimal parameter $B_{[\mu][\nu]}^{i j}$ :

$$
\begin{aligned}
& \frac{\delta \ln Z(\eta, \bar{\eta})}{\imath \delta B_{[\mu][\nu]}^{i j}}=0=\frac{1}{Z(\eta, \bar{\eta})} \int d \mu_{C}(\bar{\varphi}, \varphi)\left\{\delta_{\varphi_{[\nu]}^{j}} C_{[\mu][\alpha]}^{i l} \delta_{\bar{\varphi}_{[\alpha]}^{l}}-\delta_{\varphi_{[\alpha]}^{l}} C_{[\alpha][\nu]}^{l j} \delta_{\bar{\varphi}_{[\mu]}^{i}}-\bar{\varphi}_{[\mu]}^{i} \eta_{[\nu]}^{j}+\bar{\eta}_{[\mu]}^{i} \varphi_{[\nu]}^{j}\right. \\
& -\lambda\left[\delta^{i 0} \varphi_{[\nu]}^{j}\left[\bar{\varphi}^{1} \varphi^{2} \bar{\varphi}^{3}\right]_{[\mu]}-\delta^{j 1} \bar{\varphi}_{[\mu]}^{i}\left[\varphi^{0} \varphi^{2} \bar{\varphi}^{3}\right]_{[\nu]}+\delta^{i 2} \varphi_{[\nu]}^{j}\left[\varphi^{0} \bar{\varphi}^{1} \bar{\varphi}^{3}\right]_{[\mu]}-\delta^{j 3} \bar{\varphi}_{[\mu]}^{i}\left[\varphi^{0} \bar{\varphi}^{1} \varphi^{2}\right]_{[\nu]}\right] \\
& -\bar{\lambda}\{\bar{\varphi}\}\} e^{-S^{\operatorname{int}}(\varphi, \bar{\varphi})+\bar{\varphi} \eta+\bar{\eta} \varphi},
\end{aligned}
$$

where the new notations mean

$$
\begin{aligned}
& {\left[\bar{\varphi}^{1} \varphi^{2} \bar{\varphi}^{3}\right]_{[\mu]} }:=\int_{h_{i j}} \bar{\varphi}_{\mu_{10} h_{13} h_{12}}^{1} \varphi_{h_{21} \mu_{20} h_{23}}^{2} \bar{\varphi}_{h_{32} h_{31} \mu_{30}}^{3} \\
& {\left[\varphi^{0} \varphi^{2} \bar{\varphi}^{3}\right]_{[\nu]} }:=\int_{h_{i j}} \bar{\varphi}_{h_{03} h_{02} \nu_{01}}^{1} \varphi_{\nu_{21} h_{20} h_{23}}^{2} \bar{\varphi}_{h_{32} \nu_{31} h_{30}}^{3}, \\
& {\left[\varphi^{0} \bar{\varphi}^{1} \bar{\varphi}^{3}\right]_{[\mu]}:=\int_{h_{i j}} \varphi_{h_{03} \mu_{02} h_{01}}^{0} \bar{\varphi}_{h_{10} h_{13} \mu_{12}}^{1} \bar{\varphi}_{\mu_{32} h_{31} h_{30}}^{3}, } \\
& {\left[\varphi^{0} \bar{\varphi}^{1} \varphi^{2}\right]_{[\nu]}:=\int_{h_{i j}} \varphi_{\nu_{03} h_{02} h_{01}}^{0} \bar{\varphi}_{h_{10} \nu_{13} h_{12}}^{1} \varphi_{h_{21} h_{20} \nu_{23}}^{2} . }
\end{aligned}
$$

After some algebra (the details of which are collected in Appendix D 1), the variation of the free energy function 22 can be recast as

$$
\begin{aligned}
\frac{\delta \ln Z(\eta, \bar{\eta})}{\imath \delta B_{[\mu][\nu]}^{i j}}=0= & \frac{1}{Z(\eta, \bar{\eta})} \int d \mu_{C}(\bar{\varphi}, \varphi)\left\{-[C \bar{\varphi}]_{[b]}^{i}\left[\eta C^{\dagger}\right]_{[a]}^{j}+[C \bar{\eta}]_{[b]}^{i}\left[\varphi C^{\dagger}\right]_{[a]}^{j}\right. \\
& -\lambda\left[C_{[b][\mu]}^{i 0}\left[\varphi C^{\dagger}\right]_{[a]}^{j}\left[\bar{\varphi}^{1} \varphi^{2} \bar{\varphi}^{3}\right]_{[\mu]}-\bar{C}_{[a][\nu]}^{j 1}[C \bar{\varphi}]_{[b]}^{i}\left[\varphi^{0} \varphi^{2} \bar{\varphi}^{3}\right]_{[\nu]}\right. \\
& \left.+C_{[b][\mu]}^{i 2}\left[\varphi C^{\dagger}\right]_{[a]}^{j}\left[\varphi^{0} \bar{\varphi}^{1} \bar{\varphi}^{3}\right]_{[\mu]}-\bar{C}_{[a][\nu]}^{j 3}[C \bar{\varphi}]_{[b]}^{i}\left[\varphi^{0} \bar{\varphi}^{1} \varphi^{2}\right]_{[\nu]}\right]-\bar{\lambda}\{\bar{\varphi}\} \\
& \left.+C_{[b][\mu]}^{i i^{\prime}} \varphi_{[\mu]}^{i^{\prime}} \bar{\varphi}_{[a]}^{j}-\bar{C}_{[a][\nu]}^{j j^{\prime}} \bar{\varphi}_{[\nu]}^{j^{\prime}} \varphi_{[b]}^{i}\right\} e^{-S^{\mathrm{int}}(\varphi, \bar{\varphi})+\bar{\varphi} \eta+\bar{\eta} \varphi} .
\end{aligned}
$$

WT identity for two-point functions - The next stage is to differentiate the expression (24) using the operator ${ }^{2}$ $\left.\partial_{\eta^{p}} \partial_{\bar{\eta}^{k}}(\cdot)\right|_{\eta^{p}=\bar{\eta}^{k}=0}$ for getting the connected components of the correlation functions (we shall denote $J=\bar{\varphi} \eta+\bar{\eta} \varphi$ $\left.=\sum_{i} \bar{\varphi}^{i} \eta^{i}+\bar{\eta}^{i} \varphi^{i}\right)$

$$
\begin{aligned}
& 0=\left\langle\partial _ { \eta } \partial _ { \overline { \eta } } \left[-[C \bar{\varphi}]_{[b]}^{i}\left[\eta C^{\dagger}\right]_{[a]}^{j}+[C \bar{\eta}]_{[b]}^{i}\left[\varphi C^{\dagger}\right]_{[a]}^{j}\right.\right. \\
& -\lambda\left[C_{[b][\mu]}^{i 0}\left[\varphi C^{\dagger}\right]_{[a]}^{j}\left[\bar{\varphi}^{-1} \varphi^{2} \bar{\varphi}^{3}\right]_{[\mu]}-\bar{C}_{[a][\nu]}^{j 1}[C \bar{\varphi}]_{[b]}^{i}\left[\varphi^{0} \varphi^{2} \bar{\varphi}^{3}\right]_{[\nu]}\right.
\end{aligned}
$$

\footnotetext{
2 The indices $p, k$ are fixed in $\left.\partial_{\eta^{p}} \partial_{\bar{\eta}^{k}}(\cdot)\right|_{\eta^{p}=\bar{\eta}^{k}=0}$. Moreover, omitting for a moment these indices, $\left.\partial_{\eta^{p}} \partial_{\bar{\eta}^{k}}(\cdot)\right|_{\eta^{p}=\bar{\eta}^{k}=0}$ will be denoted by
$\partial_{\eta} \partial_{\bar{\eta}}$ in the following.
} 


$$
\begin{aligned}
& \left.+C_{[b][\mu]}^{i 2}\left[\varphi C^{\dagger}\right]_{[a]}^{j}\left[\varphi^{0} \bar{\varphi}^{1} \bar{\varphi}^{3}\right]_{[\mu]}-\bar{C}_{[a][\nu]}^{j 3}[C \bar{\varphi}]_{[b]}^{i}\left[\varphi^{0} \bar{\varphi}^{1} \varphi^{2}\right]_{[\nu]}\right]-\bar{\lambda}\{\bar{\varphi}\} \\
& \left.\left.+C_{[b][\mu]}^{i i^{\prime}} \varphi_{[\mu]}^{i^{\prime}} \bar{\varphi}_{[a]}^{j}-\bar{C}_{[a][\nu]}^{j j^{\prime}} \bar{\varphi}_{[\nu]}^{j^{\prime}} \varphi_{[b]}^{i}\right]\left.e^{J}\right|_{\eta=\bar{\eta}=0}\right\rangle_{c} .
\end{aligned}
$$

A direct computation yields at first

$$
\begin{aligned}
& \left\langle\left.\left[C_{[b][\mu]}^{i i^{\prime}} \varphi_{[\mu]}^{i^{\prime}} \bar{\varphi}_{[a]}^{j}-\left[C^{\dagger}\right]_{[\nu][a]}^{j^{\prime} j} \bar{\varphi}_{[\nu]}^{j^{\prime}} \varphi_{[b]}^{i}\right] \partial_{\eta}(\bar{\varphi} \eta) \partial_{\bar{\eta}}(\bar{\eta} \varphi)\right|_{\eta=\bar{\eta}=0}\right\rangle_{c} \\
& -\lambda\left\langle\left.\left[C_{[b][\mu]}^{i 0}\left[\varphi C^{\dagger}\right]_{[a]}^{j}\left[\bar{\varphi}^{1} \varphi^{2} \bar{\varphi}^{3}\right]_{[\mu]}\right] \partial_{\eta}(\bar{\varphi} \eta) \partial_{\bar{\eta}}(\bar{\eta} \varphi)\right|_{\eta=\bar{\eta}=0}\right\rangle_{c}+\lambda\left\langle\left.\left[\left[C^{\dagger}\right]_{[\nu][a]}^{1 j}[C \bar{\varphi}]_{[b]}^{i}\left[\varphi^{0} \varphi^{2} \bar{\varphi}^{3}\right]_{[\nu]}\right] \partial_{\eta}(\bar{\varphi} \eta) \partial_{\bar{\eta}}(\bar{\eta} \varphi)\right|_{\eta=\bar{\eta}=0}\right\rangle_{c} \\
& -\lambda\left\langle\left.\left[C_{[b][\mu]}^{i 2}\left[\varphi C^{\dagger}\right]_{[a]}^{j}\left[\varphi^{0} \bar{\varphi}^{1} \bar{\varphi}^{3}\right]_{[\mu]}\right] \partial_{\eta}(\bar{\varphi} \eta) \partial_{\bar{\eta}}(\bar{\eta} \varphi)\right|_{\eta=\bar{\eta}=0}\right\rangle_{c}+\lambda\left\langle\left.\left[\left[C^{\dagger}\right]_{[\nu][a]}^{3 j}[C \bar{\varphi}]_{[b]}^{i}\left[\varphi^{0} \bar{\varphi}^{1} \varphi^{2}\right]_{[\nu]}\right] \partial_{\eta}(\bar{\varphi} \eta) \partial_{\bar{\eta}}(\bar{\eta} \varphi)\right|_{\eta=\bar{\eta}=0}\right\rangle_{c} \\
& -\bar{\lambda}\{\bar{\varphi}\}=\left\langle\left.\left[\partial_{\eta}[C \bar{\varphi}]_{[b]}^{i}\left[\eta C^{\dagger}\right]_{[a]}^{j} \partial_{\bar{\eta}}(\bar{\eta} \varphi)-\partial_{\bar{\eta}}[C \bar{\eta}]_{[b]}^{i}\left[\varphi C^{\dagger}\right]_{[a]}^{j} \partial_{\eta}(\bar{\varphi} \eta)\right]\right|_{\eta=\bar{\eta}=0}\right\rangle_{c} .
\end{aligned}
$$

Performing the explicit differentiation with respect to $\eta_{[m]}^{p}$ and $\bar{\eta}_{[n]}^{k}$, one gets

$$
\begin{aligned}
& \left\langle\left[[C \bar{\varphi}]_{[b]}^{i}\left[C^{\dagger}\right]_{[m][a]}^{p j} \varphi_{[n]}^{k}-\left[\varphi C^{\dagger}\right]_{[a]}^{j} C_{[b][n]}^{i k} \bar{\varphi}_{[m]}^{p}\right]\right\rangle_{c}= \\
& \left\langle\left[C_{[b][\mu]}^{i i^{\prime}} \varphi_{[\mu]}^{i^{\prime}} \bar{\varphi}_{[a]}^{j}-\left[C^{\dagger}\right]_{[\nu][a]}^{j^{\prime} j} \bar{\varphi}_{[\nu]}^{j^{\prime}} \varphi_{[b]}^{i}\right] \bar{\varphi}_{[m]}^{p} \varphi_{[n]}^{k}\right\rangle_{c} \\
& -\lambda\left\langle\left[C_{[b][\mu]}^{i 0}\left[\varphi C^{\dagger}\right]_{[a]}^{j}\left[\bar{\varphi}^{1} \varphi^{2} \bar{\varphi}^{3}\right]_{[\mu]}\right] \bar{\varphi}_{[m]}^{p} \varphi_{[n]}^{k}\right\rangle_{c}+\lambda\left\langle\left[\left[C^{\dagger}\right]_{[\nu][a]}^{1 j}[C \bar{\varphi}]_{[b]}^{i}\left[\varphi^{0} \varphi^{2} \bar{\varphi}^{3}\right]_{[\nu]}\right] \bar{\varphi}_{[m]}^{p} \varphi_{[n]}^{k}\right\rangle_{c} \\
& -\lambda\left\langle\left[C_{[b][\mu]}^{i 2}\left[\varphi C^{\dagger}\right]_{[a]}^{j}\left[\varphi^{0} \bar{\varphi}^{1} \bar{\varphi}^{3}\right]_{[\mu]}\right] \bar{\varphi}_{[m]}^{p} \varphi_{[n]}^{k}\right\rangle_{c}+\lambda\left\langle\left[\left[C^{\dagger}\right]_{[\nu][a]}^{3 j}[C \bar{\varphi}]_{[b]}^{i}\left[\varphi^{0} \bar{\varphi}^{1} \varphi^{2}\right]_{[\nu]}\right] \bar{\varphi}_{[m]}^{p} \varphi_{[n]}^{k}\right\rangle_{c}-\bar{\lambda}\{\bar{\varphi}\},
\end{aligned}
$$

so that summing over $[\mu]$ and $[\nu]$, the following statement holds:

Theorem 1. Two-point functions of the colored Boulatov under a generic unitary field transformation satisfy the relation, for $i, j, p, k=0,1,2,3$,

$$
\begin{aligned}
& \left\langle[C \bar{\varphi}]_{[b]}^{i}\left[C^{\dagger}\right]_{[m][a]}^{p j} \varphi_{[n]}^{k}\right\rangle_{c}-\left\langle\left[\varphi C^{\dagger}\right]_{[a]}^{j} C_{[b][n]}^{i k} \bar{\varphi}_{[m]}^{p}\right\rangle_{c}= \\
& \left\langle\bar{\varphi}_{[a]}^{j}[C \varphi]_{[b]}^{i} \bar{\varphi}_{[m]}^{p} \varphi_{[n]}^{k}\right\rangle_{c}-\left\langle\left[\bar{\varphi} C^{\dagger}\right]_{[a]}^{j} \varphi_{[b]}^{i} \bar{\varphi}_{[m]}^{p} \varphi_{[n]}^{k}\right\rangle_{c} \\
& -\lambda\left\langle\left[\varphi C^{\dagger}\right]_{[a]}^{j} i 0\left[C \cdot \bar{\varphi}^{1} \varphi^{2} \bar{\varphi}^{3}\right]_{[b]} \bar{\varphi}_{[m]}^{p} \varphi_{[n]}^{k}\right\rangle_{c}+\lambda\left\langle[C \bar{\varphi}]_{[b]}^{i}{ }^{1 j}\left[\varphi^{0} \varphi^{2} \bar{\varphi}^{3} \cdot C^{\dagger}\right]_{[a]} \bar{\varphi}_{[m]}^{p} \varphi_{[n]}^{k}\right\rangle_{c} \\
& -\lambda\left\langle\left[\varphi C^{\dagger}\right]_{[a]}^{j} i 2\left[C \cdot \varphi^{0} \bar{\varphi}^{-1} \bar{\varphi}^{3}\right]_{[b]} \bar{\varphi}_{[m]}^{p} \varphi_{[n]}^{k}\right\rangle_{c}+\lambda\left\langle[C \bar{\varphi}]_{[b]}^{i}{ }^{3 j}\left[\varphi^{0} \bar{\varphi}^{1} \varphi^{2} \cdot C^{\dagger}\right]_{[a]} \bar{\varphi}_{[m]}^{p} \varphi_{[n]}^{k}\right\rangle_{c}-\bar{\lambda}\{\bar{\varphi}\},
\end{aligned}
$$

where we introduced the notations

$$
\begin{aligned}
{ }^{i 0}\left[C \cdot \bar{\varphi}^{1} \varphi^{2} \bar{\varphi}^{3}\right]_{[a]}:=C_{[a][\mu]}^{i 0}\left[\bar{\varphi}^{1} \varphi^{2} \bar{\varphi}^{3}\right]_{[\mu]}, & { }^{1 j}\left[\varphi^{0} \varphi^{2} \bar{\varphi}^{3} \cdot C^{\dagger}\right]_{[a]}:=\left[C^{\dagger}\right]_{[\nu][a]}^{1 j}\left[\varphi^{0} \varphi^{2} \bar{\varphi}^{3}\right]_{[\nu]}, \\
{ }^{i 2}\left[C \cdot \varphi^{0} \bar{\varphi}^{1} \bar{\varphi}^{3}\right]_{[a]}:=C_{[a][\mu]}^{i 2}\left[\varphi^{0} \bar{\varphi}^{1} \bar{\varphi}^{3}\right]_{[\mu]}, & { }^{3 j}\left[\varphi^{0} \bar{\varphi}^{-1} \varphi^{2} \cdot C^{\dagger}\right]_{[a]}:=\left[C^{\dagger}\right]_{[\nu][a]}^{3 j}\left[\varphi^{0} \bar{\varphi}^{-1} \varphi^{2}\right]_{[\nu]},
\end{aligned}
$$

for which repeated indices are summed.

The case of four external colors - Let us consider that the external color labels $i, j, p, k$ are pairwise distinct. For definiteness, let us assume that $i=0, j=1, p=2$ and $k=3$, then the WT identity becomes

$$
\begin{aligned}
& 0=\left\langle\bar{\varphi}_{[a]}^{1}[C \varphi]_{[b]}^{0} \bar{\varphi}_{[m]}^{2} \varphi_{[n]}^{3}\right\rangle_{c}-\left\langle\left[\bar{\varphi} C^{\dagger}\right]_{[a]}^{1} \varphi_{[b]}^{0} \bar{\varphi}_{[m]}^{2} \varphi_{[n]}^{3}\right\rangle_{c} \\
& -\lambda\left\langle\left[\varphi C^{\dagger}\right]_{[a]}^{1} 00\left[C \cdot \bar{\varphi}^{1} \varphi^{2} \bar{\varphi}^{3}\right]_{[b]} \bar{\varphi}_{[m]}^{2} \varphi_{[n]}^{3}\right\rangle_{c}+\lambda\left\langle[C \bar{\varphi}]_{[b]}^{0}{ }^{11}\left[\varphi^{0} \varphi^{2} \bar{\varphi}^{3} \cdot C^{\dagger}\right]_{[a]} \bar{\varphi}_{[m]}^{2} \varphi_{[n]}^{3}\right\rangle_{c}-\bar{\lambda}\{\bar{\varphi}\} .
\end{aligned}
$$

This is an identity for a four-point function with four external color which might be useful in the study of the coupling constant renormalization.

The case of two external colors - Let us assume now that $i=k=0$ and $j=p=1$

$$
\begin{aligned}
& \left\langle[C \bar{\varphi}]_{[b]}^{0}\left[C^{\dagger}\right]_{[m][a]}^{11} \varphi_{[n]}^{0}\right\rangle_{c}-\left\langle\left[\varphi C^{\dagger}\right]_{[a]}^{1} C_{[b][n]}^{00} \bar{\varphi}_{[m]}^{1}\right\rangle_{c}= \\
& \left\langle\bar{\varphi}_{[a]}^{1}[C \varphi]_{[b]}^{0} \bar{\varphi}_{[m]}^{1} \varphi_{[n]}^{0}\right\rangle_{c}-\left\langle\left[\bar{\varphi} C^{\dagger}\right]_{[a]}^{1} \varphi_{[b]}^{0} \bar{\varphi}_{[m]}^{1} \varphi_{[n]}^{0}\right\rangle_{c} \\
& -\lambda\left\langle\left[\varphi C^{\dagger}\right]_{[a]}^{1}{ }^{00}\left[C \cdot \bar{\varphi}^{1} \varphi^{2} \bar{\varphi}^{3}\right]_{[b]} \bar{\varphi}_{[m]}^{1} \varphi_{[n]}^{0}\right\rangle_{c}+\lambda\left\langle[C \bar{\varphi}]_{[b]}^{0}{ }^{11}\left[\varphi^{0} \varphi^{2} \bar{\varphi}^{3} \cdot C^{\dagger}\right]_{[a]} \bar{\varphi}_{[m]}^{1} \varphi_{[n]}^{0}\right\rangle_{c}-\bar{\lambda}\{\bar{\varphi}\} .
\end{aligned}
$$


WT identities for four-point functions - To obtain higher order point functions, we derivate again the free energy. Derivating twice $\ln Z$ for computing the four-point function identities, we have

$$
\begin{aligned}
& \left\langle\left.\left[C_{[b][\mu]}^{i i^{\prime}} \varphi_{[\mu]}^{i^{\prime}} \bar{\varphi}_{[a]}^{j}-\left[C^{\dagger}\right]_{[\nu][a]}^{j^{\prime} j} \bar{\varphi}_{[\nu]}^{j^{\prime}} \varphi_{[b]}^{i}\right] \partial_{\eta_{2}} J \partial_{\bar{\eta}_{2}} J \partial_{\eta_{1}} J \partial_{\bar{\eta}_{1}} J\right|_{\eta=\bar{\eta}=0}\right\rangle_{c} \\
& -\lambda\left\langle\left.\left[C_{[b][\mu]}^{i 0}\left[\varphi C^{\dagger}\right]_{[a]}^{j}\left[\bar{\varphi}^{-1} \varphi^{2} \bar{\varphi}^{3}\right]_{[\mu]}\right] \partial_{\eta_{2}} J \partial_{\bar{\eta}_{2}} J \partial_{\eta_{1}} J \partial_{\bar{\eta}_{1}} J\right|_{\eta=\bar{\eta}=0}\right\rangle_{c} \\
& +\lambda\left\langle\left.\left[\left[C^{\dagger}\right]_{[\nu][a]}^{1 j}[C \bar{\varphi}]_{[b]}^{i}\left[\varphi^{0} \varphi^{2} \bar{\varphi}^{3}\right]_{[\nu]}\right] \partial_{\eta_{2}} J \partial_{\bar{\eta}_{2}} J \partial_{\eta_{1}} J \partial_{\bar{\eta}_{1}} J\right|_{\eta=\bar{\eta}=0\rangle_{c}}\right. \\
& -\lambda\left\langle\left.\left[C_{[b][\mu]}^{i 2}\left[\varphi C^{\dagger}\right]_{[a]}^{j}\left[\varphi^{0} \bar{\varphi}^{1} \bar{\varphi}^{3}\right]_{[\mu]}\right] \partial_{\eta_{2}} J \partial_{\bar{\eta}_{2}} J \partial_{\eta_{1}} J \partial_{\bar{\eta}_{1}} J\right|_{\eta=\bar{\eta}=0}\right\rangle_{c} \\
& +\lambda\left\langle\left.\left[\left[C^{\dagger}\right]_{[\nu][a]}^{3 j}[C \bar{\varphi}]_{[b]}^{i}\left[\varphi^{0} \bar{\varphi}^{-1} \varphi^{2}\right]_{[\nu]}\right] \partial_{\eta_{2}} J \partial_{\bar{\eta}_{2}} J \partial_{\eta_{1}} J \partial_{\bar{\eta}_{1}} J\right|_{\eta=\bar{\eta}=0}\right\rangle_{c}-\bar{\lambda}\{\bar{\varphi}\} \\
& =\left\langle\left.\left[[C \bar{\varphi}]_{[b]}^{i} \partial_{\eta_{1}}\left[\eta C^{\dagger}\right]_{[a]}^{j} \partial_{\bar{\eta}_{1}} J-\partial_{\bar{\eta}_{1}}[C \bar{\eta}]_{[b]}^{i}\left[\varphi C^{\dagger}\right]_{[a]}^{j} \partial_{\eta_{1}} J\right] \partial_{\bar{\eta}_{2}} J \partial_{\eta_{2}} J\right|_{\eta=\bar{\eta}=0}\right\rangle_{c}+(1 \leftrightarrow 2) .
\end{aligned}
$$

Fixing the indices of $\left(\eta_{1}\right)_{[m]}^{k},\left(\bar{\eta}_{1}\right)_{[n]}^{l}\left(\eta_{2}\right)_{[p]}^{t}$ and $\left(\bar{\eta}_{2}\right)_{[q]}^{s}$, the differentiations yield

$$
\begin{aligned}
& \left\langle\left[C_{[b][\mu]}^{i i^{\prime}} \varphi_{[\mu]}^{i^{\prime}} \bar{\varphi}_{[a]}^{j}-\left[C^{\dagger}\right]_{[\nu][a]}^{j^{\prime} j} \bar{\varphi}_{[\nu]}^{j^{\prime}} \varphi_{[b]}^{i}\right] \bar{\varphi}_{[p]}^{t} \varphi_{[q]}^{s} \bar{\varphi}_{[m]}^{k} \varphi_{[n]}^{l}\right\rangle_{c} \\
& -\lambda\left\langle\left[C_{[b][\mu]}^{i 0}\left[\varphi C^{\dagger}\right]_{[a]}^{j}\left[\bar{\varphi}^{1} \varphi^{2} \bar{\varphi}^{3}\right]_{[\mu]}\right] \bar{\varphi}_{[p]}^{t} \varphi_{[q]}^{s} \bar{\varphi}_{[m]}^{k} \varphi_{[n]}^{l}\right\rangle_{c} \\
& +\lambda\left\langle\left[\left[C^{\dagger}\right]_{[\nu][a]}^{1 j}[C \bar{\varphi}]_{[b]}^{i}\left[\varphi^{0} \varphi^{2} \bar{\varphi}^{3}\right]_{[\nu]}\right] \bar{\varphi}_{[p]}^{t} \varphi_{[q]}^{s} \bar{\varphi}_{[m]}^{k} \varphi_{[n]}^{l}\right\rangle_{c} \\
& -\lambda\left\langle\left[C_{[b][\mu]}^{i 2}\left[\varphi C^{\dagger}\right]_{[a]}^{j}\left[\varphi^{0} \bar{\varphi}^{1} \bar{\varphi}^{3}\right]_{[\mu]}\right] \bar{\varphi}_{[p]}^{t} \varphi_{[q]}^{s} \bar{\varphi}_{[m]}^{k} \varphi_{[n]}^{l}\right\rangle_{c} \\
& +\lambda\left\langle\left[\left[C^{\dagger}\right]_{[\nu][a]}^{3 j}[C \bar{\varphi}]_{[b]}^{i}\left[\varphi^{0} \bar{\varphi}^{1} \varphi^{2}\right]_{[\nu]}\right] \bar{\varphi}_{[p]}^{t} \varphi_{[q]}^{s} \bar{\varphi}_{[m]}^{k} \varphi_{[n]}^{l}\right\rangle_{c}-\bar{\lambda}\{\bar{\varphi}\} \\
& =\left\langle\left[[C \bar{\varphi}]_{[b]}^{i}\left[C^{\dagger}\right]_{[m][a]}^{k j} \varphi_{[n]}^{l}-C_{[b][n]}^{i l}\left[\varphi C^{\dagger}\right]_{[a]}^{j} \bar{\varphi}_{[m]}^{k}\right] \bar{\varphi}_{[p]}^{t} \varphi_{[q]}^{s}\right\rangle_{c}+[(k, l) \leftrightarrow(t, s)] .
\end{aligned}
$$

Summing over $[\mu]$ and $[\nu]$, on this last expression rests our

Theorem 2. Four-point functions of the colored Boulatov model under a generic unitary field transformation satisfy the relation, for $i, j, k, l, s, t=0,1,2,3$,

$$
\begin{aligned}
& \left\langle[C \bar{\varphi}]_{[b]}^{i} \varphi_{[n]}^{l}\left[C^{\dagger}\right]_{[m][a]}^{k j} \bar{\varphi}_{[p]}^{t} \varphi_{[q]}^{s}\right\rangle_{c}-\left\langle\left[\varphi C^{\dagger}\right]_{[a]}^{j} C_{[b][n]}^{i l} \bar{\varphi}_{[m]}^{k} \bar{\varphi}_{[p]}^{t} \varphi_{[q]}^{s}\right\rangle_{c}+[(k, l) \leftrightarrow(t, s)]= \\
& \left\langle\bar{\varphi}_{[a]}^{j}[C \varphi]_{[b]}^{i} \bar{\varphi}_{[p]}^{t} \varphi_{[q]}^{s} \bar{\varphi}_{[m]}^{k} \varphi_{[n]}^{l}\right\rangle_{c}-\left\langle\left[\bar{\varphi} C^{\dagger}\right]_{[a]}^{j} \varphi_{[b]}^{i} \bar{\varphi}_{[p]}^{t} \varphi_{[q]}^{s} \bar{\varphi}_{[m]}^{k} \varphi_{[n]}^{l}\right\rangle_{c} \\
& -\lambda\left\langle\left[\varphi C^{\dagger}\right]_{[a]}^{j} i 0\left[C \cdot \bar{\varphi}^{1} \varphi^{2} \bar{\varphi}^{3}\right]_{[b]} \bar{\varphi}_{[p]}^{t} \varphi_{[q]}^{s} \bar{\varphi}_{[m]}^{k} \varphi_{[n]}^{l}\right\rangle_{c}+\lambda\left\langle[C \bar{\varphi}]_{[b]}^{i}{ }^{1 j}\left[\varphi^{0} \varphi^{2} \bar{\varphi}^{3} \cdot C^{\dagger}\right]_{[a]} \bar{\varphi}_{[p]}^{t} \varphi_{[q]}^{s} \bar{\varphi}_{[m]}^{k} \varphi_{[n]}^{l}\right\rangle_{c} \\
& -\lambda\left\langle\left[\varphi C^{\dagger}\right]_{[a]}^{j} i 2\left[C \cdot \varphi^{0} \bar{\varphi}^{1} \bar{\varphi}^{3}\right]_{[b]} \bar{\varphi}_{[p]}^{t} \varphi_{[q]}^{s} \bar{\varphi}_{[m]}^{k} \varphi_{[n]}^{l}\right\rangle_{c}+\lambda\left\langle[C \bar{\varphi}]_{[b]}^{i} 3 j\left[\varphi^{0} \bar{\varphi}^{1} \varphi^{2} \cdot C^{\dagger}\right]_{[a]} \bar{\varphi}_{[p]}^{t} \varphi_{[q]}^{s} \bar{\varphi}_{[m]}^{k} \varphi_{[n]}^{l}\right\rangle_{c}-\bar{\lambda}\{\bar{\varphi}\} .
\end{aligned}
$$

WT identities for even-point functions - The WT identities for $[n=2 p \geq 2]$-point functions can be deduced by simple recursion from the aforementioned equations. We first need to introduce some notations

$$
\begin{array}{ll}
\mathcal{F}=[C \bar{\varphi}]_{[b]}^{i}\left[\eta C^{\dagger}\right]_{[a]}^{j}-[C \bar{\eta}]_{[b]}^{i}\left[\varphi C^{\dagger}\right]_{[a]}^{j}, & \mathcal{H}=\mathcal{F} e^{J}, \\
\partial_{\bar{\eta}_{k}} \partial_{\eta_{k}} \mathcal{H}=\left(\mathcal{F}_{k}+\left(\partial_{\bar{\eta}_{k}} J\right)\left(\partial_{\eta_{k}} J\right) \mathcal{F}\right) e^{J}, & \mathcal{F}_{k}=[C \bar{\varphi}]_{[b]}^{i} \partial_{\eta_{k}}\left[\eta C^{\dagger}\right]_{[a]}^{j}\left(\partial_{\bar{\eta}_{k}} J\right)-\partial_{\bar{\eta}_{k}}[C \bar{\eta}]_{[b]}^{i}\left[\varphi C^{\dagger}\right]_{[a]}^{j}\left(\partial_{\eta_{k}} J\right) .
\end{array}
$$

It is by simple recursion that one proves that

$$
\begin{aligned}
& {\left[\prod_{l=1}^{n} \partial_{\eta_{l}} \partial_{\bar{\eta}_{l}}\right] \mathcal{H}=\left[\sum_{k=1}^{n} \mathcal{F}_{k} \prod_{l \neq k}^{n}\left[\partial_{\eta_{l}} J \partial_{\bar{\eta}_{l}} J\right]+\prod_{l=1}^{n}\left[\partial_{\eta_{l}} J \partial_{\bar{\eta}_{l}} J\right] \mathcal{F}\right] e^{J},} \\
& {\left.\left[\prod_{l}^{n} \partial_{\eta_{l}} \partial_{\bar{\eta}_{l}}\right] \mathcal{H}\right|_{\eta=\bar{\eta}=0}=\left.\sum_{k=1}^{n} \mathcal{F}_{k} \prod_{l \neq k}^{n}\left[\partial_{\eta_{l}} J \partial_{\bar{\eta}_{l}} J\right]\right|_{\eta=\bar{\eta}=0} .}
\end{aligned}
$$

From the last line (37), we explicitly obtain by fixing the derivative with respect to the indices such that $\left(\eta_{l}\right)_{\left[a_{l}\right]}^{\left.\alpha_{l}\right]}$ and $\left(\bar{\eta}_{l}\right)_{\left[b_{l}\right]}^{\beta_{l}}$ :

$$
\left.\left[\prod_{l=1}^{n} \partial_{\eta_{l}} \partial_{\bar{\eta}_{l}}\right] \mathcal{H}\right|_{\eta=\bar{\eta}=0}=\sum_{k=1}^{n}\left([C \bar{\varphi}]_{[b]}^{i}\left[C^{\dagger}\right]_{\left[a_{k}\right][a]}^{\alpha_{k} j} \varphi_{\left[b_{k}\right]}^{\beta_{k}}-C_{[b]\left[b_{k}\right]}^{i \beta_{k}}\left[\varphi C^{\dagger}\right]_{[a]}^{j} \bar{\varphi}_{\left[a_{k}\right]}^{\alpha_{k}}\right) \prod_{l \neq k}^{n}\left[\bar{\varphi}_{\left[a_{l}\right]}^{\alpha_{l}} \varphi_{\left[b_{l}\right]}^{\beta_{l}}\right]
$$


The generalized WT identity for an even-point function can be written as

$$
\begin{aligned}
& \left\langle\left[C_{[b][\mu]}^{i i^{\prime}} \varphi_{[\mu]}^{i^{\prime}} \bar{\varphi}_{[a]}^{j}-\left[C^{\dagger}\right]_{[\nu][a]}^{j^{\prime} j} \bar{\varphi}_{[\nu]}^{j^{\prime}} \varphi_{[b]}^{i}\right] \prod_{l=1}^{n}\left[\bar{\varphi}_{\left[a_{l}\right]}^{\alpha_{l}} \varphi_{\left[b_{l}\right]}^{\beta_{l}}\right]\right\rangle_{c} \\
& -\lambda\left\langle\left[C_{[b][\mu]}^{i 0}\left[\varphi C^{\dagger}\right]_{[a]}^{j}\left[\bar{\varphi}^{-1} \varphi^{2} \bar{\varphi}^{3}\right]_{[\mu]}\right] \prod_{l=1}^{n}\left[\bar{\varphi}_{\left[a_{l}\right]}^{\alpha_{l}} \varphi_{\left[b_{l}\right]}^{\beta_{l}}\right]\right\rangle_{c} \\
& +\lambda\left\langle\left[\left[C^{\dagger}\right]_{[\nu][a]}^{1 j}[C \bar{\varphi}]_{[b]}^{i}\left[\varphi^{0} \varphi^{2} \bar{\varphi}^{3}\right]_{[\nu]}\right] \prod_{l=1}^{n}\left[\bar{\varphi}_{\left[a_{l}\right]}^{\alpha_{l}} \varphi_{\left[b_{l}\right]}^{\beta_{l}}\right]\right\rangle_{c} \\
& -\lambda\left\langle\left[C_{[b][\mu]}^{i 2}\left[\varphi C^{\dagger}\right]_{[a]}^{j}\left[\varphi^{0} \bar{\varphi}^{1} \bar{\varphi}^{3}\right]_{[\mu]}\right] \prod_{l=1}^{n}\left[\bar{\varphi}_{\left[a_{l}\right]}^{\alpha_{l}} \varphi_{\left[b_{l}\right]}^{\beta_{l}}\right]\right\rangle_{c} \\
& +\lambda\left\langle\left[\left[C^{\dagger}\right]_{[\nu][a]}^{3 j}[C \bar{\varphi}]_{[b]}^{i}\left[\varphi^{0} \bar{\varphi}^{1} \varphi^{2}\right]_{[\nu]}\right] \prod_{l=1}^{n}\left[\bar{\varphi}_{\left[a_{l}\right]}^{\alpha_{l}} \varphi_{\left[b_{l}\right]}^{\beta_{l}}\right]\right\rangle_{c}-\bar{\lambda}\{\bar{\varphi}\} \\
& =\sum_{k=1}^{n}\left\langle\left[[C \bar{\varphi}]_{[b]}^{i}\left[C^{\dagger}\right]_{\left[a_{k}\right][a]}^{\alpha_{k} j} \varphi_{\left[b_{k}\right]}^{\beta_{k}}-C_{[b]\left[b_{k}\right]}^{i \beta_{k}}\left[\varphi C^{\dagger}\right]_{[a]}^{j} \bar{\varphi}_{\left[a_{k}\right]}^{\alpha_{k}}\right] \prod_{l \neq k}^{n}\left[\bar{\varphi}_{\left[a_{l}\right]}^{\alpha_{l}} \varphi_{\left[b_{l}\right]}^{\beta_{l}}\right]\right\rangle_{c} .
\end{aligned}
$$

Summing over the remaining running indices $[\mu]$ and $[\nu]$, we have, in the same anterior notations, the following statement:

Theorem 3. Even n-point functions of the colored Boulatov model under a generic unitary field transformation satisfy the relation

$$
\begin{aligned}
& \sum_{k=1}^{n}\left\{\left\langle[C \bar{\varphi}]_{[b]}^{i}\left[C^{\dagger}\right]_{\left[a_{k}\right][a]}^{\alpha_{k} j} \varphi_{\left[b_{k}\right]}^{\beta_{k}} \prod_{\ell \neq k}^{n}\left[\bar{\varphi}_{\left[a_{\ell}\right]}^{\alpha_{\ell}} \varphi_{\left[b_{\ell}\right]}^{\beta_{\ell}}\right]\right\rangle_{c}-\left\langle C_{[b]\left[b_{k}\right]}^{i \beta_{k}}\left[\varphi C^{\dagger}\right]_{[a]}^{j} \bar{\varphi}_{\left[a_{k}\right]}^{\alpha_{k}} \prod_{\ell \neq k}^{n}\left[\bar{\varphi}_{\left[a_{\ell}\right]}^{\alpha_{\ell}} \varphi_{\left[b_{\ell}\right]}^{\beta_{\ell}}\right]\right\rangle_{c}\right\} \\
= & \left\langle\bar{\varphi}_{[a]}^{j}[C \varphi]_{[b]}^{i} \prod_{\ell=1}^{n}\left[\bar{\varphi}_{\left[a_{\ell}\right]}^{\alpha_{\ell}} \varphi_{\left[b_{\ell}\right]}^{\beta_{\ell}}\right]\right\rangle_{c}-\left\langle\left[\bar{\varphi} C^{\dagger}\right]_{[a]}^{j} \varphi_{[b]}^{i} \prod_{\ell=1}^{n}\left[\bar{\varphi}_{\left[a_{\ell}\right]}^{\alpha_{\ell}} \varphi_{\left[b_{\ell}\right]}^{\beta_{\ell}}\right]\right\rangle_{c} \\
- & \lambda\left\langle\left[\varphi C^{\dagger}\right]_{[a]}^{j}{ }^{i 0}\left[C \cdot \bar{\varphi}^{1} \varphi^{2} \bar{\varphi}^{3}\right]_{[b]} \prod_{\ell=1}^{n}\left[\bar{\varphi}_{\left[a_{\ell}\right]}^{\alpha_{\ell}} \varphi_{\left[b_{\ell}\right]}^{\beta_{\ell}}\right]\right\rangle_{c}+\lambda\left\langle[C \bar{\varphi}]_{[b]}^{i}{ }^{1 j}\left[\varphi^{0} \varphi^{2} \bar{\varphi}^{3} \cdot C^{\dagger}\right]_{[a]} \prod_{\ell=1}^{n}\left[\bar{\varphi}_{\left[a_{\ell}\right]}^{\alpha_{\ell}} \varphi_{\left[b_{\ell}\right]}^{\beta_{\ell}}\right]\right\rangle_{c} \\
- & \lambda\left\langle\left[\varphi C^{\dagger}\right]_{[a]}^{j i 2}\left[C \cdot \varphi^{0} \bar{\varphi}^{1} \bar{\varphi}^{3}\right]_{[b]} \prod_{\ell=1}^{n}\left[\bar{\varphi}_{\left[a_{\ell}\right]}^{\alpha_{\ell}} \varphi_{\left[b_{\ell}\right]}^{\beta_{\ell}}\right]\right\rangle_{c}+\lambda\left\langle[C \bar{\varphi}]_{[b]}^{i}{ }^{3 j}\left[\varphi^{0} \bar{\varphi}^{1} \varphi^{2} \cdot C^{\dagger}\right]_{[a]} \prod_{\ell=1}^{n}\left[\bar{\varphi}_{\left[a_{\ell}\right]}^{\alpha_{\ell}} \varphi_{\left[b_{\ell}\right]}^{\beta_{\ell}}\right]\right\rangle_{c}-\bar{\lambda}\{\bar{\varphi}\} .
\end{aligned}
$$

The WT identity (40) is valid in full generality regarding a generic unitary field transformation without requiring that transformation to be a symmetry of the action. We can make the following striking observation: the equation (24) (and hence the general WT identity (40)) generates without ambiguity the WT identities associated with independent non identically distributed matrix models with invertible covariances as the models studied in [30, 31. Indeed, forgetting the color index and restricting all tensors to matrices, one has just to use the facts that, on one hand, these models are covariant under a unitary symmetry in order to cancel the interaction terms in $\lambda, \bar{\lambda}$ and, on the other hand, to invert two extra covariances such that (24) and (40) generate the corresponding WT equations for these more simple cases. Hence, it is often useful to specify which kind of WT identities could be inferred from the same reasoning with now a well defined symmetry of the model. It is the main purpose of the remaining of this paper.

\section{B. Ward-Takahashi identities for the 1-action symmetry}

We begin with the partition function (11) under infinitesimal transformations given by $\delta_{B} S^{\text {int }}=0,219$ and 20 , that we symbolically write

$$
Z(\bar{\eta}, \eta)=\int d \mu_{C}(\bar{\varphi}, \varphi)\left(1-\imath \bar{\varphi} B \eta+\bar{\eta} B \varphi+\imath \delta_{\varphi}[B C-C B] \delta_{\bar{\varphi}}\right) e^{-S^{\mathrm{int}}(\varphi, \bar{\varphi})+\bar{\varphi} \eta+\bar{\eta} \varphi} .
$$

Derivating the free energy with respect to $\imath B_{\mu \nu}$ yields

$$
\begin{aligned}
\frac{\ln Z(\eta, \bar{\eta})}{\imath \delta B_{\mu \nu}}= & 0=\frac{1}{Z(\eta, \bar{\eta})} \int d \mu_{C}(\bar{\varphi}, \varphi) \int_{g_{\alpha} h_{\beta}}\left(-\bar{\varphi}_{\mu .}^{0,2} \eta_{\nu .}^{0,2}+\bar{\eta}_{\mu .}^{0,2} \varphi_{\nu .}^{0,2}-\bar{\varphi}_{. \mu}^{1,3} \eta_{. \nu}^{1,3}+\bar{\eta}_{. \mu}^{1,3} \varphi_{. \nu}^{1,3}\right. \\
& +\frac{\delta}{\delta \varphi_{\nu .}^{0,2}} C_{\mu ; ; h_{1} h_{2} h_{3}}^{0,2} \frac{\delta}{\delta \bar{\varphi}_{h_{0} h_{1} h_{2}}^{0,2}}-\frac{\delta}{\delta \varphi_{h_{0} h_{1} h_{2}}^{0,2}} C_{h_{0} h_{1} h_{2} ; \nu \cdot \frac{\delta}{\delta \bar{\varphi}_{\mu .}^{0,2}}}^{0,2}
\end{aligned}
$$




$$
\left.+\frac{\delta}{\delta \varphi_{. \nu}^{1,3}} C_{. \mu ; h_{1} h_{2} h_{3}}^{1,3} \frac{\delta}{\delta \bar{\varphi}_{h_{0} h_{1} h_{2}}^{1,3}}-\frac{\delta}{\delta \varphi_{h_{0} h_{1} h_{2}}^{1,3}} C_{h_{0} h_{1} h_{2} ; . \nu}^{1,3} \frac{\delta}{\delta \bar{\varphi}_{. \mu}^{1,3}}\right) e^{-S^{\mathrm{int}}(\varphi, \bar{\varphi})+\bar{\varphi} \eta+\bar{\eta} \varphi},
$$

where repeated indices and $(\cdot)$ arguments (called henceforth dot arguments) are summed. The latter expression can be computed to (see Appendix D2 for derivations and notations)

$$
\begin{aligned}
0= & \frac{1}{Z(\eta, \bar{\eta})} \int d \mu_{C}(\bar{\varphi}, \varphi)\left[-\bar{\varphi}_{\mu .}^{0,2} \eta_{\nu .}^{0,2}+\bar{\eta}_{\mu .}^{0,2} \varphi_{\nu .}^{0,2}-\bar{\varphi}_{. \mu}^{1,3} \eta_{. \nu}^{1,3}+\bar{\eta}_{. \mu}^{1,3} \varphi_{. \nu}^{1,3}+\varphi_{\mu .}^{0,2} \bar{\eta}_{\nu .}^{0,2}-\bar{\varphi}_{\nu .}^{0,2} \eta_{\mu .}^{0,2}+\varphi_{. \mu}^{1,3} \bar{\eta}_{. \nu}^{1,3}-\bar{\varphi}_{. \nu}^{1,3} \eta_{. \mu}^{1,3}\right. \\
& \left.-\lambda \varphi_{\mu .}^{0,2} \cdot\left[\bar{\varphi}^{1} \varphi^{2,0} \bar{\varphi}^{3}\right]_{\nu .}-\bar{\lambda} \varphi_{. \mu}^{1,3} \cdot\left[\bar{\varphi}^{0} \bar{\varphi}^{2} \varphi^{3,1}\right]_{. \nu}+\lambda \bar{\varphi}_{\nu .}^{0,2} \cdot\left[\varphi^{1} \bar{\varphi}^{2,0} \varphi^{3}\right]_{\mu .}+\bar{\lambda} \bar{\varphi}_{. \nu}^{1,3} \cdot\left[\varphi^{0} \varphi^{2} \bar{\varphi}^{3,1}\right]_{. \mu}\right] e^{-S^{\text {int }}(\varphi, \bar{\varphi})+\bar{\varphi} \eta+\bar{\eta} \varphi}
\end{aligned}
$$

and therefore, differentiating by an even product of $\eta_{\left[a_{\ell}\right]}^{\alpha_{\ell}} \bar{\eta}_{\left[b_{\ell}\right]}^{\beta_{\ell}}$, we can readily identify the WT identities as given by the equation

$$
\begin{aligned}
& \sum_{k=1}^{n}\left\{\left\langle\bar{\varphi}_{\mu .}^{0,2}[\delta]_{\left[a_{k}\right] \nu .}^{\alpha_{k}, 0,2} \varphi_{\left[b_{k}\right]}^{\beta_{k}} \prod_{\ell \neq k}^{n}\left[\bar{\varphi}_{\left[a_{\ell}\right]}^{\alpha_{\ell}} \varphi_{\left[b_{\ell}\right]}^{\beta_{\ell}}\right]\right\rangle_{c}-\left\langle[\delta]_{\left[b_{k}\right] \mu .}^{\beta_{k}, 0,2} \varphi_{\nu .}^{0,2} \bar{\varphi}_{\left[a_{k}\right]}^{\alpha_{k}} \prod_{\ell \neq k}^{n}\left[\bar{\varphi}_{\left[a_{\ell}\right]}^{\alpha_{\ell}} \varphi_{\left[b_{\ell}\right]}^{\beta_{\ell}}\right]\right\rangle_{c}\right. \\
& -\left\langle\varphi_{\mu .}^{0,2}[\delta]_{\left[b_{k}\right] \nu .}^{\beta_{k}, 0,2} \bar{\varphi}_{\left[a_{k}\right]}^{\alpha_{k}} \prod_{\ell \neq k}^{n}\left[\bar{\varphi}_{\left[a_{\ell}\right]}^{\alpha_{\ell}} \varphi_{\left[b_{\ell}\right]}^{\beta_{\ell}}\right]\right\rangle_{c}+\left\langle[\delta]_{\left[a_{k}\right] \mu .}^{\alpha_{k}, 0,2} \bar{\varphi}_{\nu .}^{0,2} \varphi_{\left[b_{k}\right]}^{\beta_{k}} \prod_{\ell \neq k}^{n}\left[\bar{\varphi}_{\left[a_{\ell}\right]}^{\alpha_{\ell}} \varphi_{\left[b_{\ell}\right]}^{\beta_{\ell}}\right]\right\rangle_{c} \\
& +\left\langle\bar{\varphi}_{. \mu}^{1,3}[\delta]_{\left[a_{k}\right] . \nu}^{\alpha_{k}, 1,3} \varphi_{\left[b_{k}\right]}^{\beta_{k}} \prod_{\ell \neq k}^{n}\left[\bar{\varphi}_{\left[a_{\ell}\right]}^{\alpha_{\ell}} \varphi_{\left[b_{\ell}\right]}^{\beta_{\ell}}\right]\right\rangle_{c}-\left\langle[\delta]_{. \mu\left[b_{k}\right]}^{\beta_{k}, 1,3} \varphi_{. \nu}^{1,3} \bar{\varphi}_{\left[a_{k}\right]}^{\alpha_{k}} \prod_{\ell \neq k}^{n}\left[\bar{\varphi}_{\left[a_{\ell}\right]}^{\alpha_{\ell}} \varphi_{\left[b_{\ell}\right]}^{\beta_{\ell}}\right]\right\rangle_{c} \\
& \left.-\left\langle\varphi_{. \mu}^{1,3}[\delta]_{\left[b_{k}\right] . \nu}^{\beta_{k}, 1,3} \bar{\varphi}_{\left[a_{k}\right]}^{\alpha_{k}} \prod_{\ell \neq k}^{n}\left[\bar{\varphi}_{\left[a_{\ell}\right]}^{\alpha_{\ell}} \varphi_{\left[b_{\ell}\right]}^{\beta_{\ell}}\right]\right\rangle_{c}+\left\langle[\delta]_{. \mu\left[a_{k}\right]}^{\alpha_{k}, 1,3} \bar{\varphi}_{. \nu}^{1,3} \varphi_{\left[b_{k}\right]}^{\beta_{k}} \prod_{\ell \neq k}^{n}\left[\bar{\varphi}_{\left[a_{\ell}\right]}^{\alpha_{\ell}} \varphi_{\left[b_{\ell}\right]}^{\beta_{\ell}}\right]\right\rangle_{c}\right\} \\
& =-\lambda\left\langle\varphi_{\mu .}^{0,2} \cdot\left[\bar{\varphi}^{1} \varphi^{2,0} \bar{\varphi}^{3}\right]_{\nu .} \prod_{\ell=1}^{n}\left[\bar{\varphi}_{\left[a_{\ell}\right]}^{\alpha_{\ell}} \varphi_{\left[b_{\ell}\right]}^{\beta_{\ell}}\right]\right\rangle_{c}-\bar{\lambda}\left\langle\varphi_{. \mu}^{1,3} \cdot\left[\bar{\varphi}^{0} \bar{\varphi}^{2} \varphi^{3,1}\right]_{. \nu} \prod_{\ell=1}^{n}\left[\bar{\varphi}_{\left[a_{\ell}\right]}^{\alpha_{\ell}} \varphi_{\left[b_{\ell}\right]}^{\beta_{\ell}}\right]\right\rangle_{c} \\
& +\lambda\left\langle\bar{\varphi}_{\nu \cdot}^{0,2} \cdot\left[\varphi^{1} \bar{\varphi}^{2,0} \varphi^{3}\right]_{\mu .} \prod_{\ell=1}^{n}\left[\bar{\varphi}_{\left[a_{\ell}\right]}^{\alpha_{\ell}} \varphi_{\left[b_{\ell}\right]}^{\beta_{\ell}}\right]\right\rangle_{c}+\bar{\lambda}\left\langle\bar{\varphi}_{. \nu}^{1,3} \cdot\left[\varphi^{0} \varphi^{2} \bar{\varphi}^{3,1}\right]_{. \mu} \prod_{\ell=1}^{n}\left[\bar{\varphi}_{\left[a_{\ell}\right]}^{\alpha_{\ell}} \varphi_{\left[b_{\ell}\right]}^{\beta_{\ell}}\right]\right\rangle_{c},
\end{aligned}
$$

where $[\delta]_{[a][b]}^{\alpha, i, j}=\left(\delta^{\alpha, i}+\delta^{\alpha, j}\right) \delta_{[a][b]}$. This leads us to the following statement.

Theorem 4. Even n-point functions of the colored Boulatov model under a right invariant unitary transformation satisfy the relation

$$
\begin{aligned}
& \sum_{k=1}^{n}\left\{\sum _ { \alpha = 0 , 2 } \left[\delta^{\alpha_{k} \alpha} \delta_{a_{k 1} \nu}\left\langle\bar{\varphi}_{\mu a_{k 2} a_{k 3}}^{\alpha} \varphi_{\left[b_{k}\right]}^{\beta_{k}} \prod_{\ell \neq k}^{n}\left[\bar{\varphi}_{\left[a_{\ell}\right]}^{\alpha_{\ell}} \varphi_{\left[b_{\ell}\right]}^{\left.\beta_{\ell}\right]}\right]\right\rangle_{c}-\delta^{\alpha \beta_{k}} \delta_{\mu b_{k 1}}\left\langle\varphi_{\nu b_{k 2} b_{k 3}}^{\alpha} \bar{\varphi}_{\left[a_{k}\right]}^{\alpha_{k}} \prod_{\ell \neq k}^{n}\left[\bar{\varphi}_{\left[a_{\ell}\right]}^{\alpha_{\ell}} \varphi_{\left[b_{\ell}\right]}^{\beta_{\ell}}\right]\right\rangle_{c}\right.\right. \\
& \left.-\delta^{\alpha \beta_{k}} \delta_{b_{k 1} \nu}\left\langle\varphi_{\mu b_{k 2} b_{k 3}}^{\alpha} \bar{\varphi}_{\left[a_{k}\right]}^{\alpha_{k}} \prod_{\ell \neq k}^{n}\left[\bar{\varphi}_{\left[a_{\ell}\right]}^{\alpha_{\ell}} \varphi_{\left[b_{\ell}\right]}^{\beta_{\ell}}\right]\right\rangle_{c}+\delta^{\alpha \alpha_{k}} \delta_{\mu a_{k 1}}\left\langle\bar{\varphi}_{\nu a_{k 2} a_{k 3}}^{\alpha} \varphi_{\left[b_{k}\right]}^{\beta_{k}} \prod_{\ell \neq k}^{n}\left[\bar{\varphi}_{\left[a_{\ell}\right]}^{\alpha_{\ell}} \varphi_{\left[b_{\ell}\right]}^{\beta_{\ell}}\right]\right\rangle_{c}\right] \\
& +\sum_{\alpha=1,3}\left[\delta^{\alpha_{k} \alpha} \delta_{a_{k 3} \nu}\left\langle\bar{\varphi}_{a_{k 1} a_{k 2} \mu}^{\alpha} \varphi_{\left[b_{k}\right]}^{\beta_{k}} \prod_{\ell \neq k}^{n}\left[\bar{\varphi}_{\left[a_{\ell}\right]}^{\alpha_{\ell}} \varphi_{\left[b_{\ell}\right]}^{\beta_{\ell}}\right]\right\rangle_{c}-\delta^{\alpha \beta_{k}} \delta_{\mu b_{k 3}}\left\langle\varphi_{b_{k 1} b_{k 2} \nu}^{\alpha} \bar{\varphi}_{\left[a_{k}\right]}^{\alpha_{k}} \prod_{\ell \neq k}^{n}\left[\bar{\varphi}_{\left[a_{\ell}\right]}^{\alpha_{\ell}} \varphi_{\left[b_{\ell}\right]}^{\beta_{\ell}}\right]\right\rangle_{c}\right. \\
& \left.\left.-\delta^{\alpha \beta_{k}} \delta_{b_{k 3} \nu}\left\langle\varphi_{b_{k 1} b_{k 2} \mu}^{\alpha} \bar{\varphi}_{\left[a_{k}\right]}^{\alpha_{k}} \prod_{\ell \neq k}^{n}\left[\bar{\varphi}_{\left[a_{\ell}\right]}^{\alpha_{\ell}} \varphi_{\left[b_{\ell}\right]}^{\beta_{\ell}}\right]\right\rangle_{c}+\delta^{\alpha \alpha_{k}} \delta_{\mu a_{k 3}}\left\langle\bar{\varphi}_{a_{k 1} a_{k 2} \nu}^{\alpha_{\left[b_{k}\right]}} \prod_{\ell \neq k}^{\beta_{k}}\left[\bar{\varphi}_{\left[a_{\ell}\right]}^{\alpha_{\ell}} \varphi_{\left[b_{\ell}\right]}^{\beta_{\ell}}\right]\right\rangle_{c}\right]\right\} \\
& =\lambda \sum_{\alpha=0,2}\left[-\left\langle\varphi_{\mu .}^{\alpha} \cdot\left[\bar{\varphi}^{1} \varphi^{\bar{\alpha}} \bar{\varphi}^{3}\right]_{\nu \cdot} \prod_{\ell=1}^{n}\left[\bar{\varphi}_{\left[a_{\ell}\right]}^{\alpha_{\ell}} \varphi_{\left[b_{\ell}\right]}^{\beta_{\ell}}\right]\right\rangle_{c}+\left\langle\bar{\varphi}_{\nu \cdot}^{\alpha} \cdot\left[\varphi^{1} \bar{\varphi}^{\bar{\alpha}} \varphi^{3}\right]_{\mu} . \prod_{\ell=1}^{n}\left[\bar{\varphi}_{\left[a_{\ell}\right]}^{\alpha_{\ell}} \varphi_{\left[b_{\ell}\right]}^{\beta_{\ell}}\right]\right\rangle_{c}\right] \\
& +\bar{\lambda} \sum_{\alpha=1,3}\left[-\left\langle\varphi_{. \mu}^{\alpha} \cdot\left[\bar{\varphi}^{0} \bar{\varphi}^{2} \varphi^{\bar{\alpha}}\right]_{. \nu} \prod_{\ell=1}^{n}\left[\bar{\varphi}_{\left[a_{\ell}\right]}^{\alpha_{\ell}} \varphi_{\left[b_{\ell}\right]}^{\beta_{\ell}}\right]\right\rangle_{c}+\left\langle\bar{\varphi}_{\cdot \nu}^{\alpha} \cdot\left[\varphi^{0} \varphi^{2} \bar{\varphi}^{\bar{\alpha}}\right]_{. \mu} \prod_{\ell=1}^{n}\left[\bar{\varphi}_{\left[a_{\ell}\right]}^{\alpha_{\ell}} \varphi_{\left[b_{\ell}\right]}^{\beta_{\ell}}\right]\right\rangle_{c}\right],
\end{aligned}
$$

where $\bar{\alpha}=2,0$ if $\alpha=0,2$, respectively, and $\bar{\alpha}=3,1$ if $\alpha=1,3$, respectively, and in the right hand side of the equality, the notations explicitly mean

$$
\begin{aligned}
& \varphi_{\mu \cdot}^{0} \cdot\left[\bar{\varphi}^{1} \varphi^{2} \bar{\varphi}^{3}\right]_{\nu .}:=\int_{h g h_{i j}} \varphi_{\mu h g}^{0} \bar{\varphi}_{g h_{13} h_{12}}^{1} \varphi_{h_{21} h h_{23}}^{2} \bar{\varphi}_{h_{32} h_{31} \nu}^{3}, \\
& \varphi_{\mu .}^{2} \cdot\left[\bar{\varphi}^{1} \varphi^{0} \bar{\varphi}^{3}\right]_{\nu .}:=\int_{h g h_{i j}} \varphi_{h_{03} h h_{01}}^{0} \bar{\varphi}_{h_{10} h_{13} \nu}^{1} \varphi_{\mu h g}^{2} \bar{\varphi}_{g h_{31} h_{30}}^{3}
\end{aligned}
$$




$$
\begin{aligned}
\varphi_{. \mu}^{1} \cdot\left[\bar{\varphi}^{0} \bar{\varphi}^{2} \varphi^{3}\right]_{. \nu} & :=\int_{h g h_{i j}} \bar{\varphi}_{h_{03} h_{02} h}^{0} \varphi_{h g \mu}^{1} \bar{\varphi}_{\nu h_{20} h_{23}}^{2} \varphi_{h_{32} g h_{30}}^{3}, \\
\varphi_{. \mu}^{3} \cdot\left[\bar{\varphi}^{0} \bar{\varphi}^{2} \varphi^{1}\right]_{. \nu} & :=\int_{h g h_{i j}} \bar{\varphi}_{\nu h_{02} h_{01}}^{0} \varphi_{h_{10} g h_{12}}^{1} \bar{\varphi}_{h_{21} h_{20} h}^{2} \varphi_{h g \mu}^{3},
\end{aligned}
$$

and the analogous for $\bar{\varphi}_{\nu .}^{0,2} \cdot\left[\varphi^{1} \bar{\varphi}^{2,0} \varphi^{3}\right]_{\mu}$. and $\bar{\varphi}_{. \nu}^{1,3} \cdot\left[\varphi^{0} \varphi^{2} \bar{\varphi}^{3,1}\right]_{. \mu} \cdot$

At the first sight, one may wonder why Theorem 3 looks simpler than Theorem 4 . This is really an illusion because, in the second case, the symmetry constrains much more the equality and fewer terms will survive. From the general WT identity 45, we can derive some more specific relations characterizing particular graphs. Let us for instance discuss the case of two-point graphs. For this category of graphs, 45 simplifies to

$$
\begin{aligned}
& \sum_{\alpha=0,2}\left[\delta^{\alpha_{0} \alpha} \delta_{a_{1} \nu}\left\langle\bar{\varphi}_{\mu a_{2} a_{3}}^{\alpha} \varphi_{[b]}^{\beta_{0}}\right\rangle_{c}-\delta^{\alpha \beta_{0}} \delta_{\mu b_{1}}\left\langle\varphi_{\nu b_{2} b_{3}}^{\alpha} \bar{\varphi}_{[a]}^{\alpha_{0}}\right\rangle_{c}\right. \\
& \left.-\delta^{\alpha \beta_{0}} \delta_{b_{1} \nu}\left\langle\varphi_{\mu b_{2} b_{3}}^{\alpha} \bar{\varphi}_{[a]}^{\alpha_{0}}\right\rangle_{c}+\delta^{\alpha \alpha_{0}} \delta_{\mu a_{1}}\left\langle\bar{\varphi}_{\nu a_{2} a_{3}}^{\alpha} \varphi_{[b]}^{\beta_{0}}\right\rangle_{c}\right]_{c} \\
& +\sum_{\alpha=1,3}\left[\delta^{\alpha_{0} \alpha} \delta_{a_{3} \nu}\left\langle\bar{\varphi}_{a_{1} a_{2} \mu}^{\alpha} \varphi_{[b]}^{\beta_{0}}\right\rangle_{c}-\delta^{\alpha \beta_{0}} \delta_{\mu b_{3}}\left\langle\varphi_{b_{1} b_{2} \nu}^{\alpha} \bar{\varphi}_{[a]}^{\alpha_{0}}\right\rangle_{c}\right. \\
& \left.-\delta^{\alpha \beta_{0}} \delta_{b_{3} \nu}\left\langle\varphi_{b_{1} b_{2} \mu}^{\alpha} \bar{\varphi}_{[a]}^{\alpha_{0}}\right\rangle_{c}+\delta^{\alpha \alpha_{0}} \delta_{\mu a_{3}}\left\langle\bar{\varphi}_{a_{1} a_{2} \nu}^{\alpha} \varphi_{[b]}^{\beta_{0}}\right\rangle_{c}\right]_{c} \\
& =-\lambda\left\langle\varphi_{\mu .}^{0,2} \cdot\left[\bar{\varphi}^{1} \varphi^{2,0} \bar{\varphi}^{3}\right]_{\nu .} \bar{\varphi}_{[a]}^{\alpha_{0}} \varphi_{[b]}^{\beta_{0}}\right\rangle_{c}-\bar{\lambda}\left\langle\varphi_{. \mu}^{1,3} \cdot\left[\bar{\varphi}^{0} \bar{\varphi}^{2} \varphi^{3,1}\right]_{. \nu} \bar{\varphi}_{[a]}^{\alpha_{0}} \varphi_{[b]}^{\beta_{0}}\right\rangle_{c} \\
& +\lambda\left\langle\bar{\varphi}_{\nu .}^{0,2} \cdot\left[\varphi^{1} \bar{\varphi}^{2,0} \varphi^{3}\right]_{\mu .} \bar{\varphi}_{[a]}^{\alpha_{0}} \varphi_{[b]}^{\beta_{0}}\right\rangle_{c}+\bar{\lambda}\left\langle\bar{\varphi}_{. \nu}^{1,3} \cdot\left[\varphi^{0} \varphi^{2} \bar{\varphi}^{3,1}\right]_{. \mu} \bar{\varphi}_{[a]}^{\alpha_{0}} \varphi_{[b]}^{\beta_{0}}\right\rangle_{c} .
\end{aligned}
$$

where a sum is performed repeated color indices and on dot arguments whereas $[a]$ and $[b]$ are kept fixed. Using a minimal symmetry, $\varphi^{0} \rightarrow{ }^{U} \varphi^{0}$ and $\varphi^{3} \rightarrow{ }^{U} \varphi^{3}$, which makes again $S^{\text {int }}=U S^{\text {int }}$, the whole analysis gets simplified further. We then assume that only remains terms involving $\alpha=0$ and 3 , then the above WT identity can be recast in the following way

$$
\begin{aligned}
& \delta^{\alpha_{0} 0} \delta_{a_{1} \nu}\left\langle\bar{\varphi}_{\mu a_{2} a_{3}}^{0} \varphi_{[b]}^{\beta_{0}}\right\rangle_{c}-\delta^{0 \beta_{0}} \delta_{\mu b_{1}}\left\langle\varphi_{\nu b_{2} b_{3}}^{0} \bar{\varphi}_{[a]}^{\alpha_{0}}\right\rangle_{c}-\delta^{0 \beta_{0}} \delta_{b_{1} \nu}\left\langle\varphi_{\mu b_{2} b_{3}}^{0} \bar{\varphi}_{[a]}^{\alpha_{0}}\right\rangle_{c}+\delta^{0 \alpha_{0}} \delta_{\mu a_{1}}\left\langle\bar{\varphi}_{\nu a_{2} a_{3}}^{0} \varphi_{[b]}^{\beta_{0}}\right\rangle_{c} \\
& +\delta^{\alpha_{0} 3} \delta_{a_{3} \nu}\left\langle\bar{\varphi}_{a_{1} a_{2} \mu}^{3} \varphi_{[b]}^{\beta_{0}}\right\rangle_{c}-\delta^{3 \beta_{0}} \delta_{\mu b_{3}}\left\langle\varphi_{b_{1} b_{2} \nu}^{3} \bar{\varphi}_{[a]}^{\alpha_{0}}\right\rangle_{c}-\delta^{3 \beta_{0}} \delta_{b_{3} \nu}\left\langle\varphi_{b_{1} b_{2} \mu}^{3} \bar{\varphi}_{[a]}^{\alpha_{0}}\right\rangle_{c}+\delta^{3 \alpha_{0}} \delta_{\mu a_{3}}\left\langle\bar{\varphi}_{a_{1} a_{2} \nu}^{3} \varphi_{[b]}^{\beta_{0}}\right\rangle_{c} \\
= & -\lambda\left\langle\varphi_{\mu \cdot}^{0} \cdot\left[\bar{\varphi}^{1} \varphi^{2} \bar{\varphi}^{3}\right]_{\nu .} \bar{\varphi}_{[a]}^{\alpha_{0}} \varphi_{[b]}^{\beta_{0}}\right\rangle_{c}-\bar{\lambda}\left\langle\varphi_{. \mu}^{3} \cdot\left[\bar{\varphi}^{0} \bar{\varphi}^{2} \varphi^{1}\right]_{. \nu} \bar{\varphi}_{[a]}^{\alpha_{0}} \varphi_{[b]}^{\beta_{0}}\right\rangle_{c} \\
+ & \lambda\left\langle\bar{\varphi}_{\nu \cdot}^{0} \cdot\left[\varphi^{1} \bar{\varphi}^{2} \varphi^{3}\right]_{\mu .} \bar{\varphi}_{[a]}^{\alpha_{0}} \varphi_{[b]}^{\beta_{0}}\right\rangle_{c}+\bar{\lambda}\left\langle\bar{\varphi}_{. \nu}^{3} \cdot\left[\varphi^{0} \varphi^{2} \bar{\varphi}^{1}\right]_{. \mu} \bar{\varphi}_{[a]}^{\alpha_{0}} \varphi_{[b]}^{\beta_{0}}\right\rangle_{c} .
\end{aligned}
$$

From the fact that (see 25 Lemma 2.1) in a color model, an even-point function with a color missing on the external legs has external colors appearing always in pairs $(\bar{\varphi} \varphi)$, therefore, assuming further that two-point functions do not vanish, we can require that $\alpha_{0}=\beta_{0}=03^{3}$ Taking into account these assumptions, the relation 48 becomes

$$
\begin{aligned}
& \delta_{a_{1} \nu}\left\langle\bar{\varphi}_{\mu a_{2} a_{3}}^{0} \varphi_{[b]}^{0}\right\rangle_{c}-\delta_{\mu b_{1}}\left\langle\bar{\varphi}_{[a]}^{0} \varphi_{\nu b_{2} b_{3}}^{0}\right\rangle_{c}-\delta_{b_{1} \nu}\left\langle\bar{\varphi}_{[a]}^{0} \varphi_{\mu b_{2} b_{3}}^{0}\right\rangle_{c}+\delta_{\mu a_{1}}\left\langle\bar{\varphi}_{\nu a_{2} a_{3}}^{0} \varphi_{[b]}^{0}\right\rangle_{c} \\
= & -\lambda\left\langle\varphi_{\mu \cdot}^{0} \cdot\left[\bar{\varphi}^{1} \varphi^{2} \bar{\varphi}^{3}\right]_{\nu .} \bar{\varphi}_{[a]}^{0} \varphi_{[b]}^{0}\right\rangle_{c}-\bar{\lambda}\left\langle\varphi_{. \mu}^{3} \cdot\left[\bar{\varphi}^{0} \bar{\varphi}^{2} \varphi^{1}\right]_{. \nu} \bar{\varphi}_{[a]}^{0} \varphi_{[b]}^{0}\right\rangle_{c} \\
+ & \lambda\left\langle\bar{\varphi}_{\nu \cdot}^{0} \cdot\left[\varphi^{1} \bar{\varphi}^{2} \varphi^{3}\right]_{\mu \cdot} \bar{\varphi}_{[a]}^{0} \varphi_{[b]}^{0}\right\rangle_{c}+\bar{\lambda}\left\langle\bar{\varphi}_{. \nu}^{3} \cdot\left[\varphi^{0} \varphi^{2} \bar{\varphi}^{1}\right]_{. \mu} \bar{\varphi}_{[a]}^{0} \varphi_{[b]}^{0}\right\rangle_{c} .
\end{aligned}
$$

The following cases could be studied

Case 1: $\mu=\nu=a_{1}=b_{1}$ yielding a trivial relation

$$
\begin{aligned}
& 0=-\lambda\left\langle\varphi_{\mu \cdot}^{0} \cdot\left[\bar{\varphi}^{1} \varphi^{2} \bar{\varphi}^{3}\right]_{\mu .} \bar{\varphi}_{\mu a_{2} a_{3}}^{0} \varphi_{\mu b_{2} b_{3}}^{0}\right\rangle_{c}-\bar{\lambda}\left\langle\varphi_{. \mu}^{3} \cdot\left[\bar{\varphi}^{0} \bar{\varphi}^{2} \varphi^{1}\right]_{. \mu} \bar{\varphi}_{\mu a_{2} a_{3}}^{0} \varphi_{\mu b_{2} b_{3}}^{0}\right\rangle_{c} \\
& +\lambda\left\langle\bar{\varphi}_{\mu \cdot}^{0} \cdot\left[\varphi^{1} \bar{\varphi}^{2} \varphi^{3}\right]_{\mu} \cdot \bar{\varphi}_{\mu a_{2} a_{3}}^{0} \varphi_{\mu b_{2} b_{3}}^{0}\right\rangle_{c}+\bar{\lambda}\left\langle\bar{\varphi}_{\cdot \mu}^{3} \cdot\left[\varphi^{0} \varphi^{2} \bar{\varphi}^{1}\right]_{. \mu} \bar{\varphi}_{\mu a_{2} a_{3}}^{0} \varphi_{\mu b_{2} b_{3}}^{0}\right\rangle_{c} .
\end{aligned}
$$

Case 2: $\mu \neq \nu$, and $a_{1}=\nu$ and $b_{1}=\mu$. These assumptions lead to

$$
\left\langle\bar{\varphi}_{\mu a_{2} a_{3}}^{0} \varphi_{\mu b_{1} b_{2}}^{0}\right\rangle_{c}-\left\langle\bar{\varphi}_{\nu a_{2} a_{3}}^{0} \varphi_{\nu b_{2} b_{3}}^{0}\right\rangle_{c}=0
$$

\footnotetext{
3 This is without loss of generality since the case $\alpha_{0}=\beta_{0}=3$ can be inferred by symmetry and will lead to the similar conclusion.
} 

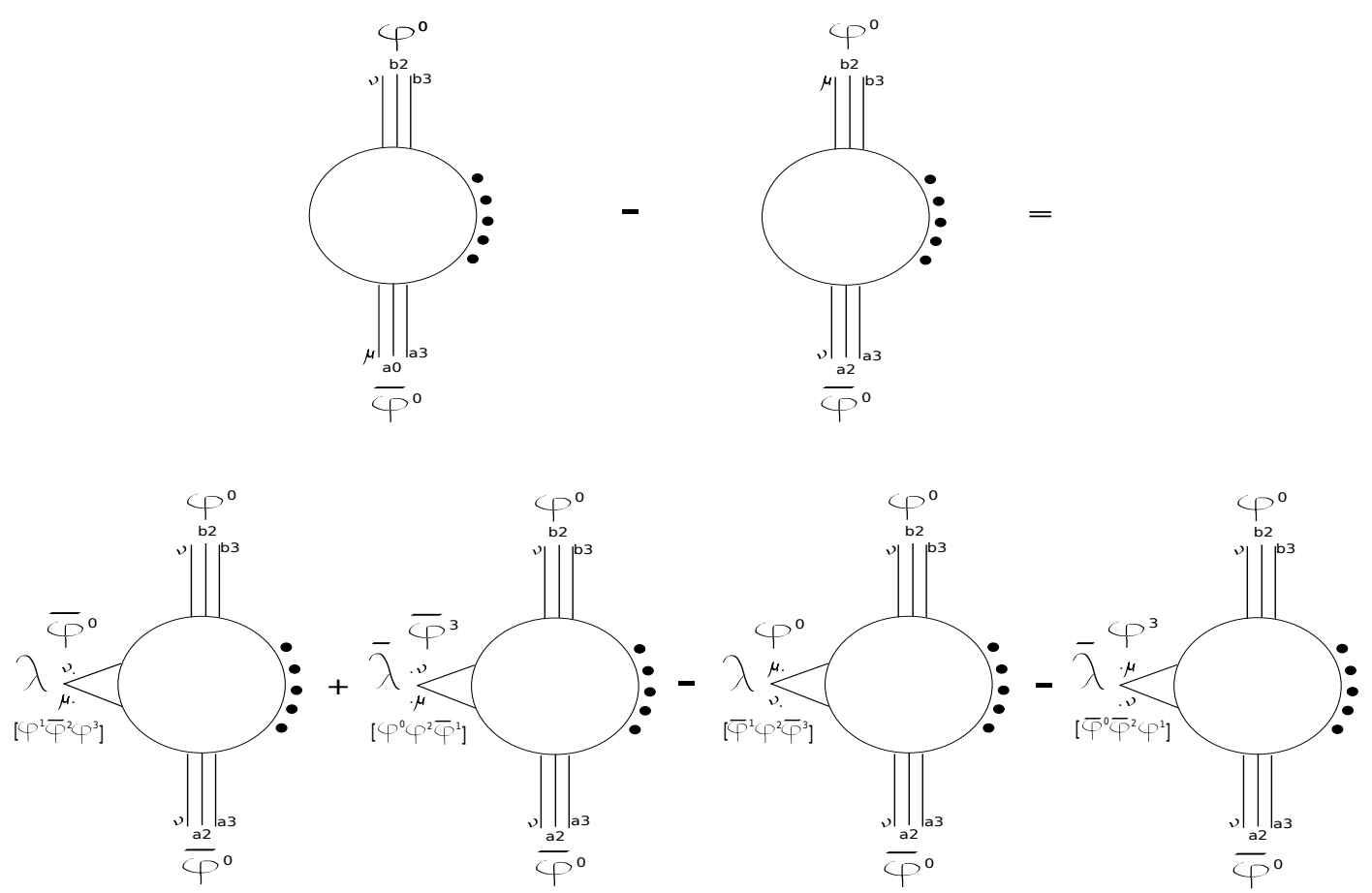

FIG. 1. Ward-Takahashi identity for the 1-action symmetry for colors 0 and 3

$$
\begin{aligned}
0 & =-\lambda\left\langle\varphi_{\mu .}^{0} \cdot\left[\bar{\varphi}^{1} \varphi^{2} \bar{\varphi}^{3}\right]_{\nu .} \bar{\varphi}_{\nu a_{2} a_{3}}^{0} \varphi_{\mu b_{1} b_{2}}^{0}\right\rangle_{c}-\bar{\lambda}\left\langle\varphi_{. \mu}^{3} \cdot\left[\bar{\varphi}^{0} \bar{\varphi}^{2} \varphi^{1}\right]_{. \nu} \bar{\varphi}_{\nu a_{2} a_{3}}^{0} \varphi_{\mu b_{1} b_{2}}^{0}\right\rangle_{c} \\
& +\lambda\left\langle\bar{\varphi}_{\nu .}^{0} \cdot\left[\varphi^{1} \bar{\varphi}^{2} \varphi^{3}\right]_{\mu .} \bar{\varphi}_{\nu a_{2} a_{3}}^{0} \varphi_{\mu b_{1} b_{2}}^{0}\right\rangle_{c}+\bar{\lambda}\left\langle\bar{\varphi}_{. \nu}^{3} \cdot\left[\varphi^{0} \varphi^{2} \bar{\varphi}^{1}\right]_{. \mu} \bar{\varphi}_{\nu a_{2} a_{3}}^{0} \varphi_{\mu b_{1} b_{2}}^{0}\right\rangle_{c},
\end{aligned}
$$

where one should use the fact that any correlation including a beginning and an end-point with the same index (for instance, $\left\langle\bar{\varphi}_{\mu a_{2} a_{3}}^{0} \varphi_{\mu b_{1} b_{2}}^{0}\right\rangle_{c}$ involving a open strand with amplitude $\left.\delta\left(\mu\left(\prod h\right) \mu^{-1}\right)\right)$ does not depend on that point (say $\mu)$. Again, this WT identity is trivial.

Case 3: $\mu \neq \nu$ and assume that $a_{1}=b_{1}=\nu$, these restrictions give

$$
\begin{aligned}
& \left\langle\bar{\varphi}_{\mu a_{2} a_{3}}^{0} \varphi_{\nu b_{2} b_{3}}^{0}\right\rangle_{c}-\left\langle\bar{\varphi}_{\nu a_{2} a_{3}}^{0} \varphi_{\mu b_{2} b_{3}}^{0}\right\rangle_{c} \\
= & -\lambda\left\langle\varphi_{\mu .}^{0} \cdot\left[\bar{\varphi}^{1} \varphi^{2} \bar{\varphi}^{3}\right]_{\nu .} \bar{\varphi}_{\nu a_{2} a_{3}}^{0} \varphi_{\nu b_{2} b_{3}}^{0}\right\rangle_{c}-\bar{\lambda}\left\langle\varphi_{\cdot \mu}^{3} \cdot\left[\bar{\varphi}^{0} \bar{\varphi}^{2} \varphi^{1}\right]_{. \nu} \bar{\varphi}_{\nu a_{2} a_{3}}^{0} \varphi_{\nu b_{2} b_{3}}^{0}\right\rangle_{c} \\
+ & \lambda\left\langle\bar{\varphi}_{\nu .}^{0} \cdot\left[\varphi^{1} \bar{\varphi}^{2} \varphi^{3}\right]_{\mu .} \bar{\varphi}_{\nu a_{2} a_{3}}^{0} \varphi_{\nu b_{2} b_{3}}^{0}\right\rangle_{c}+\bar{\lambda}\left\langle\bar{\varphi}_{. \nu}^{3} \cdot\left[\varphi^{0} \varphi^{2} \bar{\varphi}^{1}\right]_{. \mu} \bar{\varphi}_{\nu a_{2} a_{3}}^{0} \varphi_{\nu b_{2} b_{3}}^{0}\right\rangle_{c}
\end{aligned}
$$

which is a nontrivial relation. This WT identity and the kind with more external legs have been illustrated in Figure 1 where a field is graphically represented by three parallel strands each of which are associated with a field argument.

Let us find a meaning of the last equations (50), (51) and (52). The whole analysis in the paper has been performed by directly stating all computations at the quantum level by totally ignoring a possible kinetic part in some hypothetic classical action. A crucial fact is happening in the derivations having a closer look on equations (46) and (43). Let us digress a little bit in order to explain the meaning of this claim by considering instead a $3 D$ colored GFT classical model with, now, fields projected onto the gauge invariant sector of $L^{2}\left(G^{3}, \mathbb{C}\right)$ as it is in ordinary assumed in literature. Such a GFT possesses a trivial kinetic term (and so, at the quantum level, becomes an i.i.d. model) which can be written

$$
S^{\mathrm{kin}}=\sum_{a=0}^{3} \int_{[h]} \bar{\varphi}_{[h]}^{a} \varphi_{[h]}^{a}
$$


and an interaction $S^{\text {int }}$ still of the form (4). Within this framework, the equations of motion for fields are obtained by simple variation of the action. For instance, the equation of $\varphi^{0}$ can be written as $\left(\right.$ omitting the $\left.1 / \sqrt{\delta^{N}(e)}\right)$

$$
0=\bar{\varphi}_{h_{03} h_{02} h_{01}}^{0}+\lambda \int_{h_{i j}} \bar{\varphi}_{h_{10} h_{13} h_{12}}^{1} \varphi_{h_{21} h_{20} h_{23}}^{2} \bar{\varphi}_{h_{32} h_{31} h_{30}}^{3},
$$

the rest of equations for other fields can be easily inferred. Assuming that all these equations of motion for all colors hold, it is striking that all interaction terms perfectly cancel:

$$
-\lambda \varphi_{\mu .}^{0,2} \cdot\left[\bar{\varphi}^{1} \varphi^{2,0} \bar{\varphi}^{3}\right]_{\nu .}+\lambda \bar{\varphi}_{\nu .}^{0,2} \cdot\left[\varphi^{1} \bar{\varphi}^{2,0} \varphi^{3}\right]_{\mu .}=-\lambda \varphi_{\mu .}^{0,2} \cdot \bar{\varphi}_{\nu .}^{0,2}+\lambda \bar{\varphi}_{\nu .}^{0,2} \cdot \varphi_{\mu .}^{0,2}=0
$$

so that (43) reduces to simple contributions with sources. Under this light, equations (50) and (51) are of course totally trivial whereas (52) just teaches us that the full two-point function is symmetric under strand index permutation $\mu \leftrightarrow \nu$ which is true for an i.i.d model since the strand index should be conserved along an open face of amplitude $\delta\left(\mu \nu^{-1}\right)$. Hence, the non trivial relation $\sqrt{52}$ corresponds to a quantum version of the classical equation of motion (54) living in the gauge projected theory defined by (53). It is at least in that sense that one recovers what is ordinary expected from the point of view of the WT identity formalism. From this point, we may reasonably think that the identities (52) and more generally the non trivial contributions of 45 encode quantum modified versions of equations of motion for colored fields together with a weaker version of the conservation of some conserved quantities (in the gauge invariant formulation). This last statement is the purpose of a current investigation [32].

\section{CONCLUSION}

Let us recapitulate. First, the notion of unitary gauge field symmetry in (colored or not) tensor theories has been identified: it amounts to multiply the modes of the tensor field expansion via Peter-Weyl theorem by unitary matrices, and if the field possess a color (vector) index then this index could also undergo a transformation. Then, using this generic unitary field transformation, we have succeeded in finding an extension of the ordinary derivation of WT identities adapted in the present tensor situation and in computing, starting from the two-point and four-point functions, WT identities for any even-point functions of the colored Boulatov model. These relations have been proved to be more general than the one obtained in another context of non identically distributed (with invertible covariance) matrix models. Moreover, we show that there exists a particular class of operators (called right invariant unitary operators) under which the gauge invariance property of fields is preserved and the colored GFT interaction becomes invariant. This class of operators has allowed to refine the formalism and to identify WT identities associated with this symmetry with a nontrivial content. A main result of this paper is that, in a colored theory, a combination of twopoint functions can be expanded versus a peculiar class of six-point functions with a strand insertion. We reinterpret these WT identities as quantum versions of equations of motion of a GFT fully projected onto its gauge invariant sector. Let us mention that, although it is not excluded at all that one can define equivalent unitary transformations for non colored fields being still a symmetry of the interaction in non colored GFTs (for instance by using a similar prescription as in 20]), the case of non colored GFT will inexorably lead to far more complicated derivations for extracting WT identities with similar conclusions.

This work is, as it must be acknowledged, a prime sketch and mostly technical journey on quantum aspects of (unitary) symmetry on correlation functions in (non identically distributed) tensor models. Hence, it certainly deserves to be qualitatively refined by future prospects. Indeed, we obtain for a tensor theory, both the generic and for the so-called 1-action, various kind of identities of which the content remains to be clarified and, if possible, classified. The difficulty concerning GFT colored models here resides in the richness brought by the combination of the tensor structure and the gauge projector. One has to systematically check the true content of the bulk of the identities fulfilled by $N$-point functions. Diagrammatics associated to each nontrivial relations should be also determined. More relations between these identities and quantum equations of motion and conservation of Noether quantities associated with the interaction symmetry are under investigations [32. It can be underlined that classical symmetries (translations and dilatations) have been recently investigated by introducing Laplacian dynamics in GFTs 20. It has been shown that translation invariance for colored GFTs leads to a conserved quantity associated with the energy momentum tensor. It is therefore natural to ask in this dynamical context the same questions addressed in the ordinary case, namely what are the form and meaning of the WT identities for these symmetries and their relation to the Noether currents?

Another problem that one could address is whether or not WT identities give a relation between any (or specific) kind of $N$-point functions in terms of $N+4$ starting from the simplest colored situation of the 1-action minimal symmetry. In fact, this question is of relevance in attempts to solve the theory in the sense that to give explicit expressions for correlation functions. Yet another way to think about this problem, it to use other ingredients such as 
Schwinger-Dyson equations, so that WT identities might provide a closed integral equation for $N$-point correlation functions [33. In this latter work, the author have successfully found integral equations for the full renormalized planar connected two and four-point functions (the first admits perturbative solutions). Thus, a farther stage is to exploit the structure of Schwinger-Dyson in tensor models [13 in order to get a closed form equations for a particular $N$-point functions in the colored theory. Hence, the analysis performed here could be useful for both perturbative and nonperturbative renormalization.

\section{ACKNOWLEDGEMENTS}

The author is indebted to Razvan Gurau, Valentin Bonzom and Tim Koslowski for helpful discussions at various stages of this work. Research at Perimeter Institute is supported by the Government of Canada through Industry Canada and by the Province of Ontario through the Ministry of Research and Innovation.

\section{APPENDIX}

\section{Appendix A: Gaussian integration for tensor models}

In this appendix, a series of lemmas are introduced. These pertain to the properties of the GFT Gaussian measure and are extensively used in the text. We will denote the fields $\varphi_{[h]}=\varphi\left(h_{1}, h_{2}, h_{3}\right)$, and covariance associated with Feynman Gaussian measure as $C_{[g][h]}=C_{g_{1} g_{2} g_{3} ; h_{1} h_{2} h_{3}}$ and for all field, $C_{[g][h]} \varphi_{[h]}:=\int_{[h]} C_{[g][h]} \varphi_{[h]}$. In a colored theory, fields are equipped with an extra index $\varphi_{[h]}^{i}$ and the covariance reads $C_{[g][h]}^{i j}=\delta^{i j} C_{[g][h]}$. The subsequent analysis admits a straightforward generalization in any GFT dimension.

Definition 1. A Gaussian measure of covariance $C$ is defined by its non zero correlations

$$
\int d \mu_{C}(\bar{\varphi}, \varphi) \varphi_{\left[a_{1}\right]} \ldots \varphi_{\left[a_{n}\right]} \bar{\varphi}_{\left[b_{1}\right]} \ldots \bar{\varphi}_{\left[b_{n}\right]}=\sum_{\pi} \prod_{i=1}^{n} C_{\left[a_{i}\right]\left[b_{\pi(i)}\right]},
$$

where the sum is taken over all permutations $\pi$ of $n$ elements. For a colored theory, setting $d \mu_{C}\left(\bar{\varphi}^{\ell}, \varphi^{\ell}\right)=d \mu_{C}(\bar{\varphi}, \varphi)$, we have

$$
\int d \mu_{C}(\bar{\varphi}, \varphi) \varphi_{\left[a_{1}\right]}^{k_{1}} \ldots \varphi_{\left[a_{n}\right]}^{k_{1}} \bar{\varphi}_{\left[b_{1}\right]}^{j_{1}} \ldots \bar{\varphi}_{\left[b_{n}\right]}^{j_{n}}=\sum_{\pi} \prod_{i=1}^{n} C_{\left[a_{i}\right]\left[b_{\pi(i)}\right]}^{k_{i} j_{\pi(i)}} .
$$

In the following, the developments hold in general, i.e. without colors using the definition A.1. However being interested in colored theory, we will give the corresponding result in that particular instance. In order to alleviate notations, the source term will be denoted as

$$
\bar{\eta} \varphi+\bar{\varphi} \eta:=\int_{\left[h_{i}\right]}\left(\bar{\eta}_{h_{1} h_{2} h_{3}} \varphi_{h_{1} h_{2} h_{3}}+\bar{\varphi}_{h_{1} h_{2}, h_{3}} \eta_{h_{1} h_{2} h_{3}}\right) .
$$

Lemma 1. We have

$$
\int d \mu_{C}(\bar{\varphi}, \varphi) e^{\bar{\varphi} \eta+\bar{\eta} \varphi}=e^{\bar{\eta} C \eta}
$$

Proof: By direct evaluation using the Wick theorem (A.1), we obtain

$$
\int d \mu_{C}(\bar{\varphi}, \varphi) e^{\bar{\varphi} \eta+\bar{\eta} \varphi}=\sum_{n=1}^{\infty} \frac{1}{(n !)^{2}} \sum_{\pi} \prod_{i=1}^{n} \int_{\left[a_{i}\right]\left[b_{\pi(i)}\right]} \bar{\eta}_{\left[a_{i}\right]} C_{\left[a_{i}\right]\left[b_{\pi(i)}\right]} \eta_{\left[b_{\pi(i)}\right]}=\sum_{n=1}^{\infty} \frac{1}{n !}[\bar{\eta} C \eta]^{n} .
$$

Lemma 2. [Integration by parts] Introducing the functional derivative $\delta_{\varphi_{[h]}}(\cdot)=\delta(\cdot) / \delta \varphi_{[h]}$ for any functional $H(\varphi, \bar{\varphi})$, we have

$$
\int d \mu_{C}(\bar{\varphi}, \varphi)\left(\varphi_{[a]} H(\bar{\varphi}, \varphi)-C_{[a][b]} \delta_{\bar{\varphi}_{[b]}} H(\bar{\varphi}, \varphi)\right)=0 .
$$


Proof: This relation can be shown by first introducing source terms. We rewrite

$$
\begin{aligned}
& \int d \mu_{C}(\bar{\varphi}, \varphi)\left(\varphi_{[a]} H(\bar{\varphi}, \varphi)-C_{[a][b]} \delta_{\bar{\varphi}[b]} H(\bar{\varphi}, \varphi)\right) \\
& =\int d \mu_{C}(\bar{\varphi}, \varphi)\left[\left.\varphi_{[a]} H\left(\delta_{\eta}, \delta_{\bar{\eta}}\right) e^{\bar{\varphi} \eta+\bar{\eta} \varphi}\right|_{\bar{\eta}=\eta=0}-C_{[a][b]} \delta_{\bar{\varphi}[b]}\left(\left.H\left(\delta_{\eta}, \delta_{\bar{\eta}}\right) e^{\bar{\varphi} \eta+\bar{\eta} \varphi}\right|_{\bar{\eta}=\eta=0}\right)\right] \\
& =\left.H\left(\delta_{\eta}, \delta_{\bar{\eta}}\right)\left[\int d \mu_{C}(\bar{\varphi}, \varphi)\left(\varphi_{[a]}-C_{[a][b]} \eta_{[b]}\right) e^{\bar{\varphi} \eta+\bar{\eta} \varphi}\right]\right|_{\bar{\eta}=\eta=0} .
\end{aligned}
$$

The latter expression can be calculated using Lemma 1 and the fact that number of fields $\varphi$ and $\bar{\varphi}$ should be the same in order to give a nonvanishing amplitude:

$$
\begin{aligned}
& \int d \mu_{C}(\bar{\varphi}, \varphi)\left(\varphi_{[a]}-C_{[a][b]} \eta_{[b]}\right) e^{\bar{\varphi} \eta+\bar{\eta} \varphi}= \\
& \int d \mu_{C}(\bar{\varphi}, \varphi)\left(\varphi_{[a]} \sum_{n} \frac{1}{(n+1) ! n !}(\bar{\varphi} \eta)^{n+1}(\bar{\eta} \varphi)^{n}-C_{[a][b]} \eta_{[b]} \sum_{n} \frac{1}{(n !)^{2}}(\bar{\varphi} \eta)^{n}(\bar{\eta} \varphi)^{n}\right) \\
& =\sum_{n} \frac{1}{(n !)^{2}} C_{[a][b]} \eta_{[b]} n !(\bar{\eta} C \eta)^{n}-C_{[a][b]} \eta_{[b]} \sum_{n} \frac{1}{(n !)^{2}} n !(\bar{\eta} C \eta)^{n}=0 .
\end{aligned}
$$

Lemma 2 can be translated in terms of colored fields as

$$
\int d \mu_{C}(\bar{\varphi}, \varphi)\left(\varphi_{[a]}^{i} H(\bar{\varphi}, \varphi)-C_{[a][b]}^{i j} \delta_{\bar{\varphi}_{[b]}^{j}} H(\bar{\varphi}, \varphi)\right)=0 .
$$

Indeed, following step by step the previous proof, we have

$$
\begin{aligned}
& \int d \mu_{C}(\bar{\varphi}, \varphi)\left(\varphi_{[a]}^{i}-C_{[a][b]}^{i j} \eta_{[b]}^{j}\right) e^{\bar{\varphi}^{j} \eta^{j}+\bar{\eta}^{j} \varphi^{j}} \prod_{k \neq j} e^{\bar{\varphi}^{k} \eta^{k}+\bar{\eta}^{k} \varphi^{k}}= \\
& \int d \mu_{C}(\bar{\varphi}, \varphi)\left(\varphi_{[a]}^{i} \sum_{n} \frac{1}{(n+1) ! n !}\left(\bar{\varphi}^{j} \eta^{j}\right)^{n+1}\left(\bar{\eta}^{j} \varphi^{j}\right)^{n}-C_{[a][b]}^{i j} \eta_{[b]}^{j} \sum_{n} \frac{1}{(n !)^{2}}\left(\bar{\varphi}^{j} \eta^{j}\right)^{n}\left(\bar{\eta}^{j} \varphi^{j}\right)^{n}\right) \prod_{k \neq j} e^{\bar{\varphi}^{k} \eta^{k}+\bar{\eta}^{k} \varphi^{k}} \\
& =\int d \mu_{C}\left(\bar{\varphi}^{\prime}, \varphi^{\prime}\right)\left(\sum_{n} \frac{1}{(n !)^{2}} C_{[a][b]}^{i j} \eta_{[b]}^{j} n !(\bar{\eta} C \eta)^{n}-C_{[a][b]}^{i j} \eta_{[b]}^{j} \sum_{n} \frac{1}{(n !)^{2}} n !(\bar{\eta} C \eta)^{n}=0\right) \prod_{k \neq j} e^{\bar{\varphi}^{k} \eta^{k}+\bar{\eta}^{k} \varphi^{k} .}
\end{aligned}
$$

Lemma 3. Modifying the covariance $C$ for $C+A$, the following relation holds for any functional $H(\bar{\varphi}, \varphi)$ :

$$
\int d \mu_{C+A}(\bar{\varphi}, \varphi) H(\bar{\varphi}, \varphi)=\int d \mu_{C}(\bar{\varphi}, \varphi) e^{\delta_{\varphi} A \delta_{\bar{\varphi}}} H(\bar{\varphi}, \varphi) .
$$

Proof: First, one performs the expansion using again the source term and uses Lemma 1 in order to obtain

$$
\begin{aligned}
& \left.H\left(\delta_{\eta}, \delta_{\bar{\eta}}\right)\left[\int d \mu_{C+A}(\bar{\varphi}, \varphi) e^{\bar{\varphi} \eta+\bar{\eta} \varphi}\right]\right|_{\bar{\eta}=\eta=0}=H\left(\delta_{\eta}, \delta_{\bar{\eta}}\right)\left[\left.e^{\bar{\eta} C \eta} e^{\bar{\eta} A \eta}\right|_{\bar{\eta}=\eta=0}\right. \\
& =\left.H\left(\delta_{\eta}, \delta_{\bar{\eta}}\right)\left[\int d \mu_{C}(\bar{\varphi}, \varphi) e^{\bar{\eta} A \eta} e^{\bar{\varphi} \eta+\bar{\eta} \varphi}\right]\right|_{\bar{\eta}=\eta=0}=\left.\int d \mu_{C}(\bar{\varphi}, \varphi) e^{\delta_{\varphi} A \delta_{\bar{\varphi}}} H\left(\delta_{\eta}, \delta_{\bar{\eta}}\right)\left[e^{\bar{\varphi} \eta+\bar{\eta} \varphi}\right]\right|_{\bar{\eta}=\eta=0}
\end{aligned}
$$

which is the desired result.

\section{Appendix B: Unitary transformations}

\section{Left/Right invariant unitary operators}

Let us recall first some basic elements of $G=S U(2)$ representation theory. A Wigner matrix element of an $S U(2)$ group element $g$ in the representation $j$ will be denoted by $D_{m n}^{j}(g)$. Note the properties of these representation matrices given by $\bar{D}_{m n}^{j}(g)=D_{n m}^{j}\left(g^{-1}\right)=(-)^{m-n} D_{-m-n}^{j}(g)$ and $\int d g D_{m n}^{j}(g) \bar{D}_{m^{\prime} n^{\prime}}^{j^{\prime}}(g)=\left(1 / d_{j}\right) \delta^{j j^{\prime}} \delta_{m m^{\prime}} \delta_{n n^{\prime}}$, where $d_{j}=2 j+1$. In the following, we will use the symbol $\int_{h}:=\int d h$ for denoting the Haar integral with respect to the variable $h$. Dumb sums like $\sum$ without specifying the arguments mean that these sums are performed over all repeated discrete variables. 
Any function of one variable over $G$ can be expanded in modes via Peter-Weyl representation theorem as

$$
f(g)=\sum \sqrt{d_{j}} f_{m n}^{j} D_{m n}^{j}(g):=\sum_{j, m, n} \sqrt{d_{j}} f_{m n}^{j} D_{m n}^{j}(g), \quad f_{m n}^{j}=\sqrt{d_{j}} \int_{g} f(g) \bar{D}_{m n}^{j}(g) .
$$

An operator over the one variable functions is defined by a kernel

$$
U(\alpha, \beta)=\sum \sqrt{d_{j_{1}} d_{j_{2}}} U_{m_{1} n_{1} m_{2} n_{2}}^{j_{1} j_{2}} D_{m_{1} n_{1}}^{j_{1}}(\alpha) \bar{D}_{m_{2} n_{2}}^{j_{2}}(\beta), \quad \forall \alpha, \beta \in G .
$$

The normalization is justified by the fact that

$$
\begin{aligned}
U(f)(\alpha) & =\int_{h} U(\alpha, h) f(h)=\sum \sqrt{d_{j_{1}} d_{j_{2}}} U_{m_{1} n_{1} m_{2} n_{2}}^{j_{1} j_{2}} D_{m_{1} n_{1}}^{j_{1}}(\alpha) \sum \sqrt{d_{j}} f_{m n}^{j} \int_{h} \bar{D}_{m_{2} n_{2}}^{j_{2}}(h) D_{m n}^{j}(h) \\
& =\sum \sqrt{d_{j_{1}}}\left[U_{m_{1} n_{1} m_{2} n_{2}}^{j_{1} j_{2}} f_{m_{2} n_{2}}^{j_{2}}\right] D_{m_{1} n_{1}}^{j_{1}}(\alpha) .
\end{aligned}
$$

For instance, the identity operator $\mathbb{I}$ possesses the tensor components $\mathbb{I}_{m_{1} n_{1} m_{2} j_{2}}^{j_{2}}=\delta^{j_{1} j_{2}} \delta_{m_{1} m_{2}} \delta_{n_{1} n_{2}}$ and the kernel

$$
\mathbb{I}(\alpha, \beta)=\sum \sqrt{d_{j_{1}} d_{j_{2}}} \delta^{j_{1} j_{2}} \delta_{m_{1} m_{2}} \delta_{n_{1} n_{2}} D_{m_{1} n_{1}}^{j_{1}}(\alpha) \bar{D}_{m_{2} n_{2}}^{j_{2}}(\beta)=\sum_{j} d_{j} D_{m n}^{j}(\alpha) \bar{D}_{m n}^{j}(\beta)=\sum_{j} d_{j} \chi^{j}\left(\alpha \beta^{-1}\right)=\delta\left(\alpha \beta^{-1}\right) .
$$

Note that we introduce the symbol $\chi^{j}(g):=\sum_{m} D_{m m}^{j}(g)$ denoting the character of the group element $g$ in the representation $j$.

The adjoint of an operator $U$ is denoted by $U^{\dagger}$ and its kernel is defined by $\left[U^{\dagger}\right](\alpha, \beta)=\overline{U(\beta, \alpha)}$. Hence, an operator $U$ is unitary if the following relation holds

$$
\left[U^{\dagger} U\right](\alpha, \beta)=\left[U U^{\dagger}\right](\alpha, \beta)=\int_{h} U(\alpha, h) \overline{U(\beta, h)}=\mathbb{I}(\alpha, \beta)=\delta\left(\alpha \beta^{-1}\right)
$$

Furthermore, by noting that

$$
\begin{aligned}
& \sum \sqrt{d_{j_{1}} d_{j_{2}}} U_{m_{1} n_{1} m_{2} n_{2}}^{j_{1} j_{2}} D_{m_{1} n_{1}}^{j_{1}}(\alpha) \sqrt{d_{j_{1}^{\prime}} d_{j_{2}^{\prime}}} \overline{U_{m_{1}^{\prime} n_{1}^{\prime} m_{2}^{\prime} n_{2}^{\prime}}^{j_{1}^{\prime}}} \bar{D}_{m_{1}^{\prime} n_{1}^{\prime}}^{j_{1}^{\prime}}(\beta) \int_{h} \bar{D}_{m_{2} n_{2}}^{j_{2}}(h) D_{m_{2}^{\prime} n_{2}^{\prime}}^{j_{2}^{\prime}}(h) \\
& =\sum \sqrt{d_{j_{1}} d_{j_{1}^{\prime}}}\left[U_{m_{1} n_{1} m_{2} n_{2}}^{j_{1} j_{2}} \overline{U_{m_{1}^{\prime} n_{1}^{\prime} m_{2} n_{2}}^{j_{1}^{\prime} j_{2}}}\right] D_{m_{1} n_{1}}^{j_{1}}(\alpha) \bar{D}_{m_{1}^{\prime} n_{1}^{\prime}}^{j^{\prime}}(\beta),
\end{aligned}
$$

as expected, an operator is unitary if and only if

$$
\sum_{j_{2}, m_{2}, n_{2}} U_{m_{1} n_{1} m_{2} n_{2}}^{j_{1} j_{2}} \overline{U_{m_{1}^{\prime} n_{1}^{\prime} m_{2} n_{2}}^{j_{1}^{\prime} j_{2}}}=\delta^{j_{1} j_{1}^{\prime}} \delta_{m_{1} m_{1}^{\prime}} \delta_{n_{1} n_{1}^{\prime}}
$$

Among the unitary operators there exists a special class of unitaries, those invariant under left group action

$$
\forall p, \alpha, \beta \in G, \quad A(p \alpha, p \beta)=A(\alpha, \beta), \quad \int_{h} A(\alpha, h) \overline{A(\beta, h)}=\delta\left(\alpha^{-1} \beta\right) .
$$

Due to that invariance, we have

$$
\begin{aligned}
A(\alpha, \beta)= & \int_{h} A(h \alpha, h \beta)=\sum \sqrt{d_{j_{1}} d_{j_{2}}} U_{m_{1} m_{2} n_{1} n_{2}}^{j_{1} j_{2}} D_{k_{1} n_{1}}^{j_{1}}(\alpha) \bar{D}_{k_{2} n_{2}}^{j_{2}}(\beta) \int_{h} D_{m_{1} k_{1}}^{j_{1}}(h) \bar{D}_{m_{2} k_{2}}^{j_{2}}(h) \\
& =\sum U_{m_{1} m_{2} n_{1} n_{2}}^{j_{1} j_{2}} \bar{D}_{n_{1} k_{1}}^{j_{1}}\left(\alpha^{-1}\right) \bar{D}_{k_{2} n_{2}}^{j_{2}}(\beta) \delta^{j_{1} j_{2}} \delta_{m_{1} m_{2}} \delta_{k_{1} k_{2}} \\
& =\sum\left[\sum_{m_{1}} U_{m_{1} m_{1} n_{1} n_{2}}^{j_{1} j_{1}}\right] \bar{D}_{n_{1} n_{2}}^{j_{1}}\left(\alpha^{-1} \beta\right) \equiv \sum d_{j} A_{p q}^{j} D_{p q}^{j}\left(\alpha^{-1} \beta\right)
\end{aligned}
$$

where we read off $A_{p q}^{j}=\left[(-)^{p-q} / d_{j}\right] \sum_{m} U_{m m-p-q}^{j j}$. Imposing unitarity on these operators yields

$$
\begin{aligned}
& \int_{h} A(\alpha, h) \overline{A(\beta, h)}=\sum d_{j} d_{j^{\prime}} A_{p q}^{j} \overline{A_{p^{\prime} q^{\prime}}^{j^{\prime}}} D_{p r}^{j}\left(\alpha^{-1}\right) \bar{D}_{p^{\prime} r^{\prime}}^{j^{\prime}}\left(\beta^{-1}\right) \int_{h} D_{r q}^{j}(h) \bar{D}_{r^{\prime} q^{\prime}}^{j^{\prime}}(h), \\
& =\sum d_{j}\left[\sum_{q} A_{p q}^{j} \overline{A_{p^{\prime} q}^{j}}\right] D_{p r}^{j}\left(\alpha^{-1}\right) \bar{D}_{p^{\prime} r}^{j}\left(\beta^{-1}\right)=\sum d_{j}\left[\sum_{q} A_{p q}^{j} \overline{A_{p^{\prime} q}^{j}}\right] D_{p p^{\prime}}^{j}\left(\alpha^{-1} \beta\right) .
\end{aligned}
$$

Therefore the invariant unitary operators are represented by unitary matrices in each dimension $d_{j}$ of the representation

$$
\sum_{q} A_{p q}^{j} \overline{A_{p^{\prime} q}^{j}}=\delta_{p p^{\prime}}
$$


We call such an $A$ as a left invariant unitary. For right invariant unitary operator, a similar definition can be given and a little computation leads to

$$
A(\alpha, \beta)=\int_{h} A(\alpha h, \beta h)=\sum\left[\sum_{n} U_{m_{1} m_{2} n n}^{j_{1} j_{1}}\right] D_{m_{1} m_{2}}^{j_{1}}\left(\alpha \beta^{-1}\right) \equiv \sum d_{j} A_{p q}^{j} D_{p q}^{j}\left(\alpha \beta^{-1}\right),
$$

with $A_{p q}^{j}:=\left(1 / d_{j}\right) \sum_{n} U_{p q n n}^{j j}$. Further imposing unitarity yields some conditions on the coefficients $A_{p q}^{j}$ :

$$
\begin{aligned}
& \int_{h} A(\alpha, h) \overline{A(\beta, h)}=\sum d_{j} d_{j^{\prime}} A_{p q}^{j} \overline{A_{p^{\prime} q^{\prime}}^{j^{\prime}}} \int_{h} D_{p q}^{j}\left(\alpha h^{-1}\right) \overline{D_{p^{\prime} q^{\prime}}^{j}\left(\beta h^{-1}\right)} \\
& =\sum d_{j} A_{p q}^{j} \overline{A_{p^{\prime} q}^{j}} D_{p r}^{j}(\alpha) \bar{D}_{p^{\prime} r}^{j}(\beta)=\sum d_{j}\left[\sum_{q} A_{p q}^{j} \overline{A_{p^{\prime} q}^{j}}\right] D_{p p^{\prime}}^{j}\left(\alpha \beta^{-1}\right),
\end{aligned}
$$

so that $\sum_{q} A_{p q}^{j} \overline{A_{p^{\prime} q}^{j}}=\delta_{p p^{\prime}}$ which is a similar to left invariant unitary condition B.10. However, assuming that we impose that the following is unitary

$$
\int_{h} A(h, \alpha) \overline{A(h, \beta)}=\sum d_{j}\left[\sum_{p} A_{p q}^{j} \overline{A_{p q^{\prime}}^{j}}\right] D_{q^{\prime} q}^{j}\left(\beta^{-1} \alpha\right),
$$

one could get another condition on the $A_{p q}^{j}$ 's that is

$$
\sum_{p} A_{p q}^{j} \overline{A_{p q^{\prime}}^{j}}=\delta_{q q^{\prime}}
$$

\section{Unitary transformation of fields}

1-action on fields - Consider the right invariant unitary operator as detailed in Appendix B 1 which is of the form

$$
A(g, h)=\sum d_{j} A_{m n}^{j} D_{m n}^{j}\left(g h^{-1}\right) .
$$

The condition $\sum_{n} A_{m n}^{j} \bar{A}_{m^{\prime} n}^{j}=\delta_{m m^{\prime}}$, ensures that, for all $j, A^{j}$ is a unitary matrix of rank $2 j+1$, i.e. $A^{j} \in U(2 j+1)$.

Given a $D$ dimensional GFT, the 1-action of $A$ on a field means that the said field transforms with respect to its first argument, namely

$$
{ }^{A} \varphi\left(g_{1}, g_{2}, \ldots, g_{D}\right)=\int_{h} A\left(g_{1}, h\right) \varphi\left(h, g_{2}, \ldots, g_{D}\right), \quad{ }^{A} \bar{\varphi}\left(g_{1}, g_{2}, \ldots, g_{D}\right)=\int_{h} \overline{A\left(g_{1}, h\right) \varphi\left(h, g_{2}, \ldots, g_{D}\right)},
$$

this is, using the mode expansion (and equivalent in term of matrices and tensors),

$$
\begin{aligned}
& { }^{A} \varphi\left(g_{1}, g_{2}, \ldots, g_{D}\right)=\sum d_{j} \sqrt{d_{j_{1}}} A_{p q}^{j} \varphi_{m_{1} n_{1} m_{i} n_{i}}^{j_{1} j_{i}} \int_{h} D_{p q}^{j}\left(g_{1} h^{-1}\right) D_{m_{1} n_{1}}^{j_{1}}(h) \prod_{i \neq 1} \sqrt{d_{j_{i}}} D_{m_{i} n_{i}}^{j_{i}}\left(g_{i}\right) \\
& =\sum A_{m_{1} q}^{j_{1}} \varphi_{q n_{1} m_{i} n_{i}}^{j_{1} j_{i}} \sqrt{d_{j_{1}}} D_{m_{1} n_{1}}^{j_{1}}\left(g_{1}\right) \prod_{i \neq 1}\left[\sqrt{d_{j_{i}}} D_{m_{i} n_{i}}^{j_{i}}\left(g_{i}\right)\right] \\
& { }_{\bar{\varphi}}\left(g_{1}, g_{2}, \ldots, g_{D}\right)=\sum d_{j} \sqrt{d_{j_{1}}} \bar{A}_{p q}^{j} \bar{\varphi}_{m_{1} n_{1} m_{i} n_{i}}^{j_{i} j_{1}} \int_{h} \bar{D}_{p q}^{j}\left(g_{1} h^{-1}\right) \bar{D}_{m_{1} n_{1}}^{j_{1}}(h) \prod_{i \neq 1} \sqrt{d_{j_{i}}} \bar{D}_{m_{i} n_{i}}^{j_{i}}\left(g_{i}\right) \\
& =\sum \bar{A}_{m_{1} q}^{j_{1}} \bar{\varphi}_{q n_{1} m_{i} n_{i}}^{j_{i} j_{1}} \sqrt{d_{j_{1}}} \bar{D}_{m_{1} n_{1}}^{j_{1}}\left(g_{1}\right) \prod_{i \neq 1}\left[\sqrt{d_{j_{i}}} \bar{D}_{m_{i} n_{i}}^{j_{i}}\left(g_{i}\right)\right] .
\end{aligned}
$$

Hence, the modes of the transformed field ${ }^{A} \varphi$ can be related to the modes of the prime field as

$$
{ }^{A} \varphi_{m_{1} n_{1} m_{i} n_{i}}^{j_{1} j_{i}}=\sum_{p} A_{m_{1} p}^{j_{1}} \varphi_{p n_{1} m_{i} n_{i}}^{j_{1} j_{i}}, \quad A_{\bar{\varphi}_{m_{i} n_{i} m_{1} n_{1}}^{j_{i} j_{1}}}=\sum_{p} \bar{A}_{m_{1} p}^{j_{1}} \bar{\varphi}_{p n_{1} m_{i} n_{i}}^{j_{i} j_{1}}
$$

with the notable feature that only the first set of labels coined by $1, j_{1}, m_{1}$ and $n_{1}$, is actually involved under this transformation. Returning to the group formulation, this field transformation will be referred to the equivalent forms when no possible confusion may occur

$$
A_{g h} \varphi_{h .}:={ }^{A} \varphi_{g .}=\int_{h} A(g, h) \varphi(h,(.)), \quad \bar{\varphi}_{h .} A_{h g}^{-1}:={ }^{A} \bar{\varphi}_{g .}=\int_{h} \overline{A(g, h) \varphi(h,(.))} .
$$


Infinitesimal transformation - Given a right invariant unitary $A$, its component $A^{j} \in U\left(d_{j}\right)$, and therefore there exists $\left(B^{j}\right)^{\dagger}=B^{j}$ a Hermitian matrix of the same dimension $d_{j}$, such that

$$
A_{m n}^{j}=\delta_{m n}^{j}+\imath B_{m n}^{j}, \quad \bar{B}_{n m}^{j}=B_{m n}^{j} .
$$

We can expand $A$ infinitesimally at first order in $B$ :

$$
A(h, g) \simeq \sum_{j} d_{j} \sum_{m n}\left(\delta_{m n}^{j}+\imath B_{m n}^{j}\right) D_{m n}^{j}\left(h^{-1} g\right)=\delta\left(g h^{-1}\right)+\imath B(g, h) .
$$

$B$ is a Hermitian kernel in the sense that

$$
\overline{B(h, g)}=\sum_{j} d_{j} \sum_{m n} \bar{B}_{m n}^{j} \bar{D}_{m n}^{j}\left(h g^{-1}\right)=\sum_{j} d_{j} \sum_{m n} B_{n m}^{j} D_{n m}^{j}\left(g h^{-1}\right)=B(g, h) .
$$

\section{Appendix C: Calculation of infinitesimal variations under unitary transformations}

\section{General unitary transformation}

We start by considering a general unitary operator $U$ which satisfies $U_{[a][b]}^{i j} \bar{U}_{[c][b]}^{k j}=\delta^{i k} \delta_{[a][c]}$ where $[a]:=\left(a_{1}, a_{2}, a_{3}\right)$, and $\delta_{[a][b]}$ stands for the kernel of the unit operator identifying each field arguments. Appendix B2 provides a particular type of this unitary operator of the form $U^{j k}=\delta^{j k} A \otimes \mathbb{I} \otimes \mathbb{I}$ that we will discuss in detail in the next subsection. In this appendix, we assume a formal and general expression for this operator and infer the infinitesimal variations for the action, the covariance and source term.

We assume that, under $U$, the colored fields $\varphi^{i}$ transform as

$$
U \varphi_{[a]}^{i}=U_{[a][b]}^{i j} \varphi_{[b]}^{j}, \quad U \bar{\varphi}_{[a]}^{i}=\bar{\varphi}_{[b]}^{j} \bar{U}_{[a][b]}^{i j}, \quad \frac{\delta}{\delta^{U} \varphi_{[a]}^{i}}=\frac{\delta}{\delta \varphi_{[b]}^{j}}\left(U^{-1}\right)_{[b][a]}^{j i}, \quad \frac{\delta}{\delta^{U} \bar{\varphi}_{[a]}^{i}}=U_{[a][b]}^{i j} \frac{\delta}{\delta \bar{\varphi}_{[b]}^{j}} .
$$

A sum (integration on arguments and discrete sum on colors) is understood over all repeated indices. This transformation therefore mixes both colors and group arguments of the fields.

$S^{\text {int }}$ becomes after this field transformation

$$
\begin{aligned}
& U S^{\mathrm{int}}=\frac{\lambda}{\sqrt{\delta^{N}(e)}} \int_{h_{i j}} \int_{[a][b][c][d]} U_{h_{03} h_{02} h_{01}[a]}^{i_{0}} \varphi_{[a]}^{i_{0}} \bar{\varphi}_{[b]}^{i_{1}} \bar{U}_{h_{10} h_{13} h_{12}[b]}^{1 i_{1}} U_{h_{21} h_{20} h_{23}[c]}^{2 i_{2}} \varphi_{[c]}^{i_{2}} \bar{\varphi}_{[d]}^{i_{3}} \bar{U}_{h_{32} h_{31} h_{30}[d]}^{3 i_{3}} \\
& +\frac{\bar{\lambda}}{\sqrt{\delta^{N}(e)}} \int_{h_{i j}} \int_{[a][b][c][d]} \bar{\varphi}_{[a]}^{i_{0}} \bar{U}_{h^{03} h^{02} h^{01}[a]}^{0 i_{i}} \varphi_{[b]}^{i_{1}} U_{h^{10} h^{13} h^{12}[b]}^{1 i_{1}} \bar{\varphi}_{[c]}^{i_{2}} \bar{U}_{h^{21} h^{20} h^{23}[c]}^{2 i_{2}} U_{h^{32} h^{31} h^{30}[d]}^{3 i_{3}} \varphi_{[d]}^{i_{3}} .
\end{aligned}
$$

Expanding the unitary operator around the identity, one has $U_{[a][b]}^{i j}=\delta^{i j} \delta_{[a][b]}+\imath B_{[a][b]}^{i j}$, where $B$ is a Hermitian kernel i.e. ought to satisfy $\bar{B}_{[b][a]}^{i j}=B_{[a][b]}^{j i}$. At first order in $B$, the variation of the interaction part can be computed as follows:

$$
\begin{aligned}
\delta_{B} S^{\mathrm{int}}= & \frac{\imath \lambda}{\sqrt{\delta^{N}(e)}} \int_{h_{i j}}\left[-\int_{[d]} B_{[d]\left[h_{3 i}\right]}^{i_{33} 3} \varphi_{\left[h_{0 i}\right]}^{0} \bar{\varphi}_{\left[h_{1 i}\right]}^{1} \varphi_{\left[h_{2 i}\right]}^{2} \bar{\varphi}_{[d]}^{i_{3}}+\int_{[a]} B_{\left[h_{0 i}\right][a]}^{0 i_{0}} \varphi_{[a]}^{i_{0}} \bar{\varphi}_{\left[h_{1 i}\right]}^{1} \varphi_{\left[h_{2 i}\right]}^{2} \bar{\varphi}_{\left[h_{3 i}\right]}^{3}\right. \\
& \left.-\int_{[b]} B_{[b]\left[h_{1 i}\right]}^{i_{1} 1} \varphi_{\left[h_{0 i}\right]}^{0} \bar{\varphi}_{[b]}^{i_{1}} \varphi_{\left[h_{2 i}\right]}^{2} \bar{\varphi}_{\left[h_{3 i}\right]}^{3}+\int_{c} B_{\left[h_{2 i}\right][c]}^{2 i_{2}} \varphi_{\left[h_{0 i}\right]}^{0} \bar{\varphi}_{\left[h_{1 i}\right]}^{1} \varphi_{[c]}^{i_{2}} \bar{\varphi}_{\left[h_{3 i}\right]}^{3}\right] \\
& +\frac{\imath \bar{\lambda}}{\sqrt{\delta^{N}(e)}} \int_{h^{i j}}\left[\int_{[d]} B_{\left[h^{3 i}\right][d]}^{3 i_{3}} \bar{\varphi}_{\left[h^{0 i}\right]}^{0} \varphi_{\left[h^{1 i}\right]}^{1} \bar{\varphi}_{\left[h^{2 i}\right]}^{2} \varphi_{[d]}^{i_{3}}-\int_{[a]} B_{[a]\left[h^{0 i}\right]}^{i_{0} 0} \bar{\varphi}_{[a]}^{i_{0}} \varphi_{\left[h^{1 i}\right]}^{1} \bar{\varphi}_{\left[h^{2 i}\right]}^{2} \varphi_{\left[h^{3 i}\right]}^{3}\right. \\
& \left.+\int_{[b]} B_{\left[h^{1 i}\right][b]}^{1 i_{1}} \bar{\varphi}_{\left[h^{0 i}\right]}^{0} \varphi_{[b]}^{i_{1}} \bar{\varphi}_{\left[h^{2 i}\right]}^{2} \varphi_{\left[h^{3 i}\right]}^{3}-\int_{[c]} B_{[c]\left[h^{2 i}\right]}^{i_{2} 2} \bar{\varphi}_{\left[h^{0 i}\right]}^{0} \varphi_{\left[h^{1 i}\right]}^{1} \bar{\varphi}_{[c]}^{i_{2}} \varphi_{\left[h^{3 i}\right]}^{3}\right],
\end{aligned}
$$

which can be denoted compactly by

$$
\begin{aligned}
& \delta_{B} S^{\text {int }}:=\imath \lambda\left[[B \varphi]^{0} \bar{\varphi}^{1} \varphi^{2} \bar{\varphi}^{3}-\varphi^{0}[\bar{\varphi} B]^{1} \varphi^{2} \bar{\varphi}^{3}+\varphi^{0} \bar{\varphi}^{1}[B \varphi]^{2} \bar{\varphi}^{3}-\varphi^{0} \bar{\varphi}^{1} \varphi^{2}[\bar{\varphi} B]^{3}\right]+\imath \bar{\lambda}\{\bar{\varphi}\}, \\
& \bar{\lambda}\{\bar{\varphi}\}=-[\bar{\varphi} B]^{0} \varphi^{1} \bar{\varphi}^{2} \varphi^{3}+\bar{\varphi}^{0}[B \varphi]^{1} \bar{\varphi}^{2} \varphi^{3}-\bar{\varphi}^{0} \varphi^{1}[\bar{\varphi} B]^{2} \varphi^{3}+\bar{\varphi}^{0} \varphi^{1} \bar{\varphi}^{2}[B \varphi]^{3} .
\end{aligned}
$$

Meanwhile, the source terms have the infinitesimal variations

$$
\delta_{B}(\bar{\eta} \varphi+\bar{\varphi} \eta)=\sum_{i} \int_{[g]}\left({ }^{U} \bar{\varphi}_{[g]}^{i} \eta_{[g]}^{i}+\bar{\eta}_{[g]}^{i} U_{[g]}^{i}\right)-(\bar{\eta} \varphi+\bar{\varphi} \eta)
$$




$$
\begin{aligned}
& =\sum_{i} \int_{\left[g_{i j}\right][a]}\left(\left(\delta^{i j} \delta_{[g][a]}-\imath \bar{B}_{[g][a]}^{i j}\right) \bar{\varphi}_{[a]}^{j} \eta_{[g]}^{i}+\bar{\eta}_{[g]}^{i}\left(\delta^{i j} \delta_{[g][a]}+\imath B_{[g][a]}^{i j}\right) \varphi_{[a]}^{j}\right)-\sum_{i} \int_{[g]}\left(\bar{\eta}_{[g]}^{i} \varphi_{[g]}^{i}+\bar{\varphi}_{[g]}^{i} \eta_{[g]}^{i}\right) \\
& =\imath \sum_{i} \int_{[g][a]}\left(-\bar{\varphi}_{[a]}^{j} B_{[a][g]}^{j i} \eta_{[g]}^{i}+\bar{\eta}_{[g]}^{i} B_{[g][a]}^{i j} \varphi_{[a]}^{j}\right)=: \imath(-\bar{\varphi} B \eta+\bar{\eta} B \varphi) .
\end{aligned}
$$

Under (C.1), the partition function transforms according to

$$
Z(\bar{\eta}, \eta)=\int d \mu_{U C U^{-1}}(\bar{\varphi}, \varphi) e^{-S^{\mathrm{int}}\left(U \varphi, \bar{\varphi} U^{-1}\right)+\bar{\varphi} U^{-1} \eta+\bar{\eta} U \varphi} .
$$

We have used the fact that the covariance varies as

$$
\begin{aligned}
C_{[h]\left[h^{\prime}\right]}^{k k^{\prime}} & =\int d \mu_{C}\left({ }^{U} \bar{\varphi},{ }^{U} \varphi\right) \int_{[a][b]} \bar{U}_{[h][a]}^{k j} \bar{\varphi}_{[a]}^{j} U_{\left[h^{\prime}\right][b]}^{k^{\prime} j^{\prime}} \varphi_{[b]}^{j^{\prime}} \\
\bar{U}_{\left[h^{\prime}\right]\left[b^{\prime}\right]}^{k^{\prime} l^{\prime}} C_{[h]\left[h^{\prime}\right]}^{k k^{\prime}} U_{[h]\left[a^{\prime}\right]}^{k l} & =\int d \mu_{C}\left({ }^{U} \bar{\varphi},{ }^{U} \varphi\right) \int_{[a][b][h]\left[h^{\prime}\right]}^{{ }^{\prime}} U_{[h]\left[a^{\prime}\right]}^{k l} \bar{U}_{[h][a]}^{k j} U_{\left[h^{\prime}\right][b]}^{k^{\prime} j^{\prime}} \bar{U}_{\left[h^{\prime}\right]\left[b^{\prime}\right]}^{k^{\prime} l^{\prime}} \bar{\varphi}_{[a]}^{j} \varphi_{[b]}^{j^{\prime}} \\
& =\int d \mu_{C}\left({ }^{U} \bar{\varphi},{ }^{U} \varphi\right) \int_{a b} \bar{\varphi}_{\left[a^{\prime}\right]}^{l} \varphi_{\left[b^{\prime}\right]}^{l^{\prime}}:=\left[U C U^{-1}\right]_{\left[a^{\prime}\right]\left[b^{\prime}\right]}^{l l^{\prime}}:=\left[{ }^{U} C\right]_{\left[a^{\prime}\right]\left[b^{\prime}\right]}^{l l^{\prime}} .
\end{aligned}
$$

Seeking the infinitesimal variation of the covariance, one finds

$$
\begin{aligned}
{\left[U C U^{-1}\right]_{[a][b]}^{i j}-C_{[a][b]}^{i j} } & =\left(\delta^{l i} \delta_{[c][a]}+\imath B_{[c][a]}^{l i}\right) C_{[c]\left[c^{\prime}\right]}^{l l^{\prime}}\left(\delta^{j l^{\prime}} \delta_{[b]\left[c^{\prime}\right]}-\imath B_{[b]\left[c^{\prime}\right]}^{j l^{\prime}}\right)-C_{[a][b]}^{i j} \\
& =\imath\left[-C_{[a]\left[c^{\prime}\right]}^{i l^{\prime}} B_{[b]\left[c^{\prime}\right]}^{j l^{\prime}}+B_{[c][a]}^{l i} C_{[c][b]}^{l j}\right]=: \imath[B C-C B]_{[a][b]}^{i j} .
\end{aligned}
$$

\section{Right invariant unitary transformation}

Working with a right invariant unitary in the sense of the 1-action of Appendix B2, Eq. $B .19$, we get a change of variables such that

$$
\begin{aligned}
& { }^{U} \varphi_{a .}^{0}=U_{a b} \varphi_{b .}^{0}, \quad{ }^{U} \bar{\varphi}_{a .}^{0}=\bar{\varphi}_{b .}^{0}\left(U^{-1}\right)_{b a}=\bar{U}_{a b} \bar{\varphi}_{b .}^{0}, \\
& \frac{\delta}{\delta^{U} \varphi_{a .}^{0}}=\frac{\delta}{\delta \varphi_{b .}^{0}}\left(U^{-1}\right)_{b a}, \quad \frac{\delta}{\delta^{U} \bar{\varphi}_{a .}^{0}}=U_{a b} \frac{\delta}{\delta \bar{\varphi}_{b .}^{0}}, \\
& { }^{U} \varphi_{. a}^{1}=U_{a b} \varphi_{. b}^{1}, \quad{ }^{U} \bar{\varphi}_{. a}^{1}=\bar{\varphi}_{. b}^{1}\left(U^{-1}\right)_{b a}=\bar{U}_{a b} \bar{\varphi}_{. b}^{1}, \\
& \frac{\delta}{\delta^{U} \varphi_{. a}^{1}}=\frac{\delta}{\delta \varphi_{. b}^{1}}\left(U^{-1}\right)_{b a} \\
& \frac{\delta}{\delta^{U} \bar{\varphi}_{. a}^{1}}=U_{a b} \frac{\delta}{\delta \bar{\varphi}_{. b}^{1}},
\end{aligned}
$$

meanwhile colors 2 and 3 transform like 0 and 1, respectively. The subscripts $a, b$ should be considered here as a unique group element (and not a triplet) and the dot notifies the position of the remaining arguments of the field. Hence fields 0 and 2 are transformed with respect to their first argument whereas fields 1 and 3 to their last argument.

Under $U$ the term $S^{\text {int }}$ transforms as

$$
\begin{aligned}
& U S^{\mathrm{int}}=\frac{\lambda}{\sqrt{\delta^{N}(e)}} \int_{h_{i j}} \int_{a b c d} U_{h_{03} a} \varphi_{a h_{02} h_{01}}^{0} \bar{\varphi}_{h_{10} h_{13} b}^{1} \bar{U}_{h_{12} b} U_{h_{21} c} \varphi_{c h_{20} h_{23}}^{2} \bar{\varphi}_{h_{32} h_{31} d}^{3} \bar{U}_{h_{30} d} \\
& +\frac{\bar{\lambda}}{\sqrt{\delta^{N}(e)}} \int_{h_{i j}} \int_{a b c d} \bar{U}_{h^{03} a} \bar{\varphi}_{a h^{02} h^{01}}^{0} \varphi_{h^{10} h^{13} b}^{1} U_{h^{12} b} \bar{U}_{h^{21} c} \bar{\varphi}_{c h^{20} h^{23}}^{2} \varphi_{h^{32} h^{31} d}^{3} U_{h^{30} d},
\end{aligned}
$$

using the orthogonality relation the unitary operators, namely $U_{h b} \bar{U}_{h c}=\delta_{b c}$, we have after a proper renaming of variables

$$
{ }^{U} S^{\mathrm{int}}=S^{\mathrm{int}}
$$

Remark that this symmetry can be even decomposed in two minimal and independent symmetries: one performed on the couple $(0,3)$ and another one performed on $(1,2)$. Each of these latter symmetries does not modify $S^{\text {int }}$ and can be used to determine all the subsequent developments without loss of generality. These simpler symmetries can be useful to reduce the generic WT identities and to obtain particular graphical equations.

Under (C.9), the partition function undergoes the following modification:

$$
Z(\bar{\eta}, \eta)=\int d \mu_{U C U^{-1}}(\bar{\varphi}, \varphi) e^{-S^{\mathrm{int}}\left(U \varphi, \bar{\varphi} U^{-1}\right)+\bar{\varphi} U^{-1} \eta+\bar{\eta} U \varphi} .
$$

We have used the fact that the covariance transforms as follows:

$$
C_{h_{0} h_{1} h_{2} ; h_{0}^{\prime} h_{1}^{\prime} h_{2}^{\prime}}^{i i=0}=\int d \mu_{C}\left({ }^{U} \bar{\varphi},{ }^{U} \varphi\right) \int_{a b} \bar{U}_{h_{0} a} \bar{\varphi}_{a h_{1} h_{2}}^{i=0,2} U_{h_{0}^{\prime} b} \varphi_{b h_{1}^{\prime} h_{2}^{\prime}}^{i=0,2}
$$




$$
\begin{aligned}
\bar{U}_{h_{0}^{\prime} c^{\prime}} C_{h_{0} h_{1} h_{2} ; h_{0}^{\prime} h_{1}^{\prime} h_{2}^{\prime}}^{i i=0,2} U_{h_{0} c} & =\int d \mu_{C}\left({ }^{U} \bar{\varphi},{ }^{U} \varphi\right) \int_{a b h_{0} h_{0}^{\prime}} U_{h_{0} c} \bar{U}_{h_{0} a} \bar{U}_{h_{0}^{\prime} c^{\prime}} U_{h_{0}^{\prime} b} \bar{\varphi}_{a h_{1} h_{2}}^{i=0,2} \varphi_{b h_{1}^{\prime} h_{2}^{\prime}}^{i=0,2}:=\left[U C U^{-1}\right]_{c h_{1} h_{2} ; c^{\prime} h_{1}^{\prime} h_{2}^{\prime}}^{i i=0,2} \\
C_{h_{0} h_{1} h_{2} ; h_{0}^{\prime} h_{1}^{\prime} h_{2}^{\prime}}^{i i=1,3} & =\int d \mu_{C}\left({ }^{U} \bar{\varphi},{ }^{U} \varphi\right) \int_{a b} \bar{\varphi}_{h_{0} h_{1} a}^{i=1,3} \bar{U}_{h_{2} a} \varphi_{h_{0}^{\prime} h_{1}^{\prime} b}^{i=1,3} U_{h_{2}^{\prime} b} \\
\bar{U}_{h_{2}^{\prime} c^{\prime}} C_{h_{0} h_{1} h_{2} ; h_{0}^{\prime} h_{1}^{\prime} h_{2}^{\prime}}^{i i=1,3} U_{h_{2} c} & =\int d \mu_{C}\left({ }^{U} \bar{\varphi},{ }^{U} \varphi\right) \int_{a b h_{2} h_{2}^{\prime}} U_{h_{2} c} \bar{U}_{h_{2} a} \bar{U}_{h_{2}^{\prime} c^{\prime}} U_{h_{2}^{\prime} b} \bar{\varphi}_{h_{0} h_{1} a}^{i=1,3} \varphi_{h_{0}^{\prime} h_{1}^{\prime} b}^{i=1,3}:=\left[U C U^{-1}\right]_{h_{0} h_{1} c ; h_{0}^{\prime} h_{1}^{\prime} c^{\prime}}^{i=1,3}
\end{aligned}
$$

For a small $B$, we decompose $U_{a b}=\delta_{a b}+\imath B_{a b}$, hence at first order in $B$, we can explicitly check that the variation of the interaction part vanishes. After a straightforward computation, one has

$$
\begin{aligned}
& \delta_{B} S^{\mathrm{int}}=\frac{\imath \lambda}{\sqrt{\delta^{N}(e)}} \int_{h_{i j}}\left[-\int_{d} \bar{B}_{h_{30} d} \varphi_{h_{03} h_{02} h_{01}}^{0} \bar{\varphi}_{h_{10} h_{13} h_{12}}^{1} \varphi_{h_{21} h_{20} h_{23}}^{2} \bar{\varphi}_{h_{32} h_{31} d}^{3}\right. \\
& +\int_{a} B_{h_{03} a} \varphi_{a h_{02} h_{01}}^{0} \bar{\varphi}_{h_{10} h_{13} h_{12}}^{1} \varphi_{h_{21} h_{20} h_{23}}^{2} \bar{\varphi}_{h_{32} h_{31} h_{30}}^{3} \\
& -\int_{b} \bar{B}_{h_{12} b} \varphi_{h_{03} h_{02} h_{01}}^{0} \bar{\varphi}_{h_{10} h_{13} b}^{1} \varphi_{h_{21} h_{20} h_{23}}^{2} \bar{\varphi}_{h_{32} h_{31} h_{30}}^{3} \\
& \left.+\int_{c} B_{h_{21} c} \varphi_{h_{03} h_{02} h_{01}}^{0} \bar{\varphi}_{h_{10} h_{13} h_{12}}^{1} \varphi_{c h_{20} h_{23}}^{2} \bar{\varphi}_{h_{32} h_{31} h_{30}}^{3}\right] \\
& +\frac{\imath \bar{\lambda}}{\sqrt{\delta^{N}(e)}} \int_{h^{i j}}\left[\int_{d} B_{h^{30}} \varphi_{h^{03}}^{0} h^{02} h^{01} \bar{\varphi}_{h^{10}}^{1} h^{13} h^{12} \varphi_{h^{21}}^{2} h^{20} h^{23} \bar{\varphi}_{h^{32} h^{31} d}^{3}\right. \\
& -\int_{a} \bar{B}_{h^{03} a} \varphi_{a h^{02} h^{01}}^{0} \bar{\varphi}_{h^{10} h^{13} h^{12}}^{1} \varphi_{h^{21} h^{20} h^{23}}^{2} \bar{\varphi}_{h^{32} h^{31} h^{30}}^{3} \\
& +\int_{b}^{a} B_{h^{12} b} \varphi_{h^{03} h^{02} h^{01}}^{0} \bar{\varphi}_{h^{10} h^{13} b}^{1} \varphi_{h^{21} h^{20} h^{23}}^{2} \bar{\varphi}_{h^{32} h^{31} h^{30}}^{3} \\
& \left.-\int_{c} \bar{B}_{h^{21} c} \varphi_{h^{03} h^{02} h^{01}}^{0} \bar{\varphi}_{h^{10} h^{13} h^{12}}^{1} \varphi_{c h^{20} h^{23}}^{2} \bar{\varphi}_{h^{32} h^{31} h^{30}}^{3}\right] \text {, }
\end{aligned}
$$

then using the Herimiticity of the kernel $\bar{B}_{a b}=B_{b a}$ this term cancels. Thus, $\delta_{B} S^{\text {int }}=0$.

The infinitesimal variations of the covariance are given by

$$
\begin{aligned}
{\left[U C U^{-1}\right]_{[a][b]}^{i i=0,2}-C_{[a][b]}^{i i=0,2} } & =\left(\delta_{c a_{0}}+\imath B_{c a_{0}}\right) C_{c a_{1} a_{2} ; c^{\prime} b_{1} b_{2}}^{i i=0,2}\left(\delta_{c^{\prime} b_{0}}-\imath \bar{B}_{c^{\prime} b_{0}}\right)-C_{[a][b]}^{i i=0,2} \\
& =\imath\left[-C_{[a] ; c^{\prime} b_{1} b_{2}}^{i i=0,2} B_{b_{0} c^{\prime}}+B_{c a_{0}} C_{c a_{1} a_{2} ;[b]}^{i i=0,2}=: \imath[B C-C B]_{[a][b]}^{i i=0,2},\right. \\
{\left[U C U^{-1}\right]_{[a][b]}^{i i=1,3}-C_{[a][b]}^{i i=1,3} } & =\left(\delta_{c a_{2}}+\imath B_{c a_{2}}\right) C_{a_{0} a_{1} c ; b_{0} b_{1} c^{\prime}}^{i i=1,3}\left(\delta_{c^{\prime} b_{2}}-\imath \bar{B}_{c^{\prime} b_{2}}\right)-C_{[a] b]}^{i i=1,3} \\
& =\imath\left[-C_{[a] ; b_{0} b_{1} c^{\prime}}^{i i=1,3} B_{b_{2} c^{\prime}}+B_{c a_{2}} C_{a_{0} a_{1} c ;[b]}^{i i=1,3}\right]=: \imath[B C-C B]_{[a][b]}^{i i=1,3},
\end{aligned}
$$

whereas the source terms can be varied as follows

$$
\begin{aligned}
& \delta_{B}(\bar{\eta} \varphi+\bar{\varphi} \eta)=\sum_{i} \int_{[g]}\left({ }^{U} \bar{\varphi}_{[g]}^{i} \eta_{[g]}^{i}+\bar{\eta}_{[g]}^{i} U_{[g]}^{i}\right)-(\bar{\eta} \varphi+\bar{\varphi} \eta) \\
& =\imath \int_{g_{i} a}\left\{\sum_{i=0,2}\left(-\bar{\varphi}_{a .}^{i} B_{a g_{0}} \eta_{g_{0} .}^{i}+\bar{\eta}_{g_{0} .}^{i} B_{g_{0} a} \varphi_{a .}^{i}\right)+\sum_{i=1,3}\left(-\bar{\varphi}_{. a}^{i} B_{a g_{2}} \eta_{g_{2}}^{i}+\bar{\eta}_{. g_{2}}^{i} B_{g_{2} a} \varphi_{. a}^{i}\right)\right\} \\
& =: \imath(-\bar{\varphi} B \eta+\bar{\eta} B \varphi) .
\end{aligned}
$$




\section{Appendix D: Free energy evaluations}

\section{General unitary transformation}

We start by giving the variation of free energy 22 under the infinitesimal transformation generated by a general unitary operator ${ }^{4}$

$$
\begin{aligned}
& \frac{\delta \ln Z(\eta, \bar{\eta})}{\imath \delta B_{[\mu][\nu]}^{i j}}=0=\frac{1}{Z(\eta, \bar{\eta})} \int d \mu_{C}(\bar{\varphi}, \varphi)\left\{\delta_{\varphi_{[\nu]}^{j}} C_{[\mu][\alpha]}^{i l} \delta_{\bar{\varphi}_{[\alpha]}^{l}}-\delta_{\varphi_{[\alpha]}^{l}} C_{[\alpha][\nu]}^{l j} \delta_{\bar{\varphi}_{[\mu]}^{i}}-\bar{\varphi}_{[\mu]}^{i} \eta_{[\nu]}^{j}+\bar{\eta}_{[\mu]}^{i} \varphi_{[\nu]}^{j}\right. \\
& -\lambda\left[\delta^{i 0} \varphi_{[\nu]}^{j}\left[\bar{\varphi}^{1} \varphi^{2} \bar{\varphi}^{3}\right]_{[\mu]}-\delta^{j 1} \bar{\varphi}_{[\mu]}^{i}\left[\varphi^{0} \varphi^{2} \bar{\varphi}^{3}\right]_{[\nu]}+\delta^{i 2} \varphi_{[\nu]}^{j}\left[\varphi^{0} \bar{\varphi}^{1} \bar{\varphi}^{3}\right]_{[\mu]}-\delta^{j 3} \bar{\varphi}_{[\mu]}^{i}\left[\varphi^{0} \bar{\varphi}^{1} \varphi^{2}\right]_{[\nu]}\right] \\
& -\bar{\lambda}\{\bar{\varphi}\}\} e^{-S^{\mathrm{int}}(\varphi, \bar{\varphi})+\bar{\varphi} \eta+\bar{\eta} \varphi}
\end{aligned}
$$

where the remaining arguments of fields which do not appear are integrated (see (23)). Using the Hermiticity of the covariance $\bar{C}_{[b][a]}^{i j}=C_{[a][b]}^{j i}$ (as this is the two-point correlation function and the latter is a necessarily Hermitian), D.1 is again

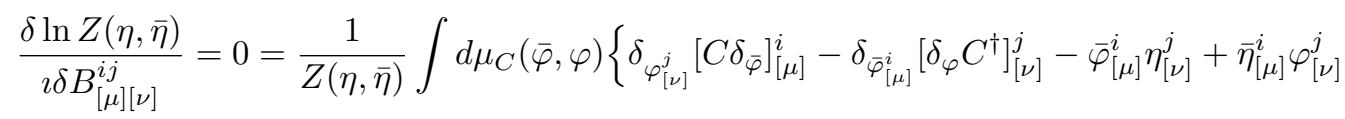

$$
\begin{aligned}
& -\lambda\left[\delta^{i 0} \varphi_{[\nu]}^{j}\left[\bar{\varphi}^{1} \varphi^{2} \bar{\varphi}^{3}\right]_{[\mu]}-\delta^{j 1} \bar{\varphi}_{[\mu]}^{i}\left[\varphi^{0} \varphi^{2} \bar{\varphi}^{3}\right]_{[\nu]}+\delta^{i 2} \varphi_{[\nu]}^{j}\left[\varphi^{0} \bar{\varphi}^{1} \bar{\varphi}^{3}\right]_{[\mu]}-\delta^{j 3} \bar{\varphi}_{[\mu]}^{i}\left[\varphi^{0} \bar{\varphi}^{1} \varphi^{2}\right]_{[\nu]}\right] \\
& -\bar{\lambda}\{\bar{\varphi}\}\} e^{-S^{\mathrm{int}}(\varphi, \bar{\varphi})+\bar{\varphi} \eta+\bar{\eta} \varphi}
\end{aligned}
$$

where $\bar{\lambda}\{\bar{\varphi}\}$ can be obtained from the term in $\lambda$ by multiplying it by $(-1)$, the symmetry $(j, \nu) \leftrightarrow(i, \mu)$ and complex conjugation.

Let us multiply D.2 and sum over repeated indices by $\bar{C}_{[a][\nu]}^{j^{\prime} j}$ and $C_{[b][\mu]}^{i^{\prime} i}$, we get, after renaming $i^{\prime}, j^{\prime}$ by $i, j$ :

$$
\begin{aligned}
& 0=\frac{1}{Z(\eta, \bar{\eta})} \int d \mu_{C}(\bar{\varphi}, \varphi)\left\{-[C \bar{\varphi}]_{[b]}^{i}\left[\eta C^{\dagger}\right]_{[a]}^{j}+[C \bar{\eta}]_{[b]}^{i}\left[\varphi C^{\dagger}\right]_{[a]}^{j}\right. \\
& -\lambda\left[C_{[b][\mu]}^{i 0}\left[\varphi C^{\dagger}\right]_{[a]}^{j}\left[\bar{\varphi}^{1} \varphi^{2} \bar{\varphi}^{3}\right]_{[\mu]}-\bar{C}_{[a][\nu]}^{j 1}\left[C \bar{\varphi}_{[b]}^{i}\left[\varphi^{0} \varphi^{2} \bar{\varphi}^{3}\right]_{[\nu]}\right.\right. \\
& \left.+C_{[b][\mu]}^{i 2}\left[\varphi C^{\dagger}\right]_{[a]}^{j}\left[\varphi^{0} \bar{\varphi}^{1} \bar{\varphi}^{3}\right]_{[\mu]}-\bar{C}_{[a][\nu]}^{j 3}[C \bar{\varphi}]_{[b]}^{i}\left[\varphi^{0} \bar{\varphi}^{1} \varphi^{2}\right]_{[\nu]}\right]-\bar{\lambda}\{\bar{\varphi}\} \\
& \left.+C_{[b][\mu]}^{i i^{\prime}}\left[\delta_{\varphi} C^{\dagger}\right]_{[a]}^{j}\left[C \delta_{\bar{\varphi}}\right]_{[\mu]}^{i^{\prime}}-\bar{C}_{[a][\nu]}^{j j^{\prime}}\left[C \delta_{\bar{\varphi}}\right]_{[b]}^{i}\left[\delta_{\varphi} C^{\dagger}\right]_{[\nu]}^{j^{\prime}}\right\} e^{-S^{\mathrm{int}}(\varphi, \bar{\varphi})+\bar{\varphi} \eta+\bar{\eta} \varphi},
\end{aligned}
$$

here, $\bar{\lambda}\{\bar{\varphi}\}$ can be obtained from the term in $\lambda$ by multiplication by $(-1)$, complex conjugation of fields and symmetry

$$
\left(i, a, \nu,\left[\varphi C^{\dagger}\right]\right) \leftrightarrow(j, b, \mu,[C \bar{\varphi}]) .
$$

Using Lemma 2, we have:

$$
\begin{aligned}
& \frac{1}{Z(\eta, \bar{\eta})} \int d \mu_{C}(\bar{\varphi}, \varphi)\left\{C_{[b][\mu]}^{i i^{\prime}}\left[\delta_{\varphi} C^{\dagger}\right]_{[a]}^{j}\left[C \delta_{\bar{\varphi}}\right]_{[\mu]}^{i^{\prime}}-\bar{C}_{[a][\nu]}^{j j^{\prime}}\left[C \delta_{\bar{\varphi}}\right]_{[b]}^{i}\left[\delta_{\varphi} C^{\dagger}\right]_{[\nu]}^{j^{\prime}}\right\} e^{-S^{\mathrm{int}}(\varphi, \bar{\varphi})+\bar{\varphi} \eta+\bar{\eta} \varphi} \\
& =\frac{1}{Z(\eta, \bar{\eta})} \int d \mu_{C}(\bar{\varphi}, \varphi)\left\{C_{[b][\mu]}^{i i^{\prime}} \bar{\varphi}_{[a]}^{j}\left[C \delta_{\bar{\varphi}}\right]_{[\mu]}^{i^{\prime}}-\bar{C}_{[a][\nu]}^{j j^{\prime}} \varphi_{[b]}^{i}\left[\delta_{\varphi} C^{\dagger}\right]_{[\nu]}^{j^{\prime}}\right\} e^{-S^{\mathrm{int}}(\varphi, \bar{\varphi})+\bar{\varphi} \eta+\bar{\eta} \varphi} .
\end{aligned}
$$

Another integration by parts and Lemma 2 yield

$$
\begin{aligned}
& \frac{1}{Z(\eta, \bar{\eta})} \int d \mu_{C}(\bar{\varphi}, \varphi)\left\{\delta_{\bar{\varphi}_{[h]}^{l}} C_{[b][\mu]}^{i i^{\prime}} \bar{\varphi}_{[a]}^{j} C_{[\mu][h]}^{i^{\prime} l}-\delta_{\bar{\varphi}_{[h]}^{l}\left[C_{[b][\mu]}^{i i^{\prime}} \bar{\varphi}_{[a]}^{j} C_{[\mu][h]}^{i^{\prime} l}\right]}\right. \\
& \left.-\delta_{\varphi_{\left[h^{\prime}\right]}^{l}} \bar{C}_{[a][\nu]}^{j j^{\prime}} \varphi_{[b]}^{i} \bar{C}_{[\nu]\left[h^{\prime}\right]}^{j^{\prime} l}+\delta_{\varphi_{\left[h^{\prime}\right]}^{l}}\left[\bar{C}_{[a][\nu]}^{j j^{\prime}} \varphi_{[b]}^{i} \bar{C}_{[\nu]\left[h^{\prime}\right]}^{j^{\prime} l}\right]\right\} e^{-S^{\text {int }}(\varphi, \bar{\varphi})+\bar{\varphi} \eta+\bar{\eta} \varphi} .
\end{aligned}
$$

Using again the fact that $C$ is Hermitian and performing some differentiations leads to

$$
\frac{1}{Z(\eta, \bar{\eta})} \int d \mu_{C}(\bar{\varphi}, \varphi)\left\{\delta_{\bar{\varphi}_{[h]}^{l}} C_{[b][\mu]}^{i i^{\prime}} \bar{\varphi}_{[a]}^{j} C_{[\mu][h]}^{i^{\prime} l}-\left[C^{2}\right]_{[b][a]}^{i j}-\delta_{\varphi_{\left[h^{\prime}\right]}^{l}} \bar{C}_{[a][\nu]}^{j j^{\prime}} \varphi_{[b]}^{i} \bar{C}_{[\nu]\left[h^{\prime}\right]}^{j^{\prime} l}+\left[\bar{C}^{2}\right]_{[a][b]}^{j i}\right\} e^{-S^{\mathrm{int}}(\varphi, \bar{\varphi})+\bar{\varphi} \eta+\bar{\eta} \varphi}(\mathrm{D}
$$

\footnotetext{
${ }^{4}$ At each step of calculation and for simplicity purpose, we will not display the term $\bar{\lambda}\{\bar{\varphi}\}$. However we will provide an explicit symmetry of its analogous, i.e. the term with coefficient $\lambda$, from which $\bar{\lambda}\{\bar{\varphi}\}$ can be determined without ambiguity.
} 
and since $\left[C^{2}\right]_{[a][b]}^{i j}=\left[\bar{C}^{2}\right]_{[b][a]}^{j i}$, these two terms cancel and the last expression (D.7) assumes now the form

$$
\frac{1}{Z(\eta, \bar{\eta})} \int d \mu_{C}(\bar{\varphi}, \varphi)\left\{C_{[b][\mu]}^{i i^{\prime}}\left[C \delta_{\bar{\varphi}}\right]_{[\mu]}^{i^{\prime}} \bar{\varphi}_{[a]}^{j}-\bar{C}_{[a][\nu]}^{j j^{\prime}}\left[\delta_{\varphi} C^{\dagger}\right]_{[\nu]}^{j^{\prime}} \varphi_{[b]}^{i}\right\} e^{-S^{\mathrm{int}}(\varphi, \bar{\varphi})+\bar{\varphi} \eta+\bar{\eta} \varphi} .
$$

Use a third time Lemma 2 in order to get

$$
\frac{1}{Z(\eta, \bar{\eta})} \int d \mu_{C}(\bar{\varphi}, \varphi)\left\{C_{[b][\mu]}^{i i^{\prime}} \varphi_{[\mu]}^{i^{\prime}} \bar{\varphi}_{[a]}^{j}-\bar{C}_{[a][\nu]}^{j j^{\prime}} \bar{\varphi}_{[\nu]}^{j^{\prime}} \varphi_{[b]}^{i}\right\} e^{-S^{\mathrm{int}}(\varphi, \bar{\varphi})+\bar{\varphi} \eta+\bar{\eta} \varphi} .
$$

Plugging this into the variation of the free energy, one obtains

$$
\begin{aligned}
& 0=\frac{1}{Z(\eta, \bar{\eta})} \int d \mu_{C}(\bar{\varphi}, \varphi)\left\{-[C \bar{\varphi}]_{[b]}^{i}\left[\eta C^{\dagger}\right]_{[a]}^{j}+[C \bar{\eta}]_{[b]}^{i}\left[\varphi C^{\dagger}\right]_{[a]}^{j}\right. \\
& -\lambda\left[C_{[b][\mu]}^{i 0}\left[\varphi C^{\dagger}\right]_{[a]}^{j}\left[\bar{\varphi}^{1} \varphi^{2} \bar{\varphi}^{3}\right]_{[\mu]}-\bar{C}_{[a][\nu]}^{j 1}[C \bar{\varphi}]_{[b]}^{i}\left[\varphi^{0} \varphi^{2} \bar{\varphi}^{3}\right]_{[\nu]}\right. \\
& \left.+C_{[b][\mu]}^{i 2}\left[\varphi C^{\dagger}\right]_{[a]}^{j}\left[\varphi^{0} \bar{\varphi}^{1} \bar{\varphi}^{3}\right]_{[\mu]}-\bar{C}_{[a][\nu]}^{j 3}[C \bar{\varphi}]_{[b]}^{i}\left[\varphi^{0} \bar{\varphi}^{1} \varphi^{2}\right]\right]-\bar{\lambda}\{\bar{\varphi}\} \\
& \left.+C_{[b][\mu]}^{i i^{\prime}} \varphi_{[\mu]}^{i^{\prime}} \bar{\varphi}_{[a]}^{j}-\bar{C}_{[a][\nu]}^{j j^{\prime}} \bar{\varphi}_{[\nu]}^{j^{\prime}} \varphi_{[b]}^{i}\right\} e^{-S^{\mathrm{int}}(\varphi, \bar{\varphi})+\bar{\varphi} \eta+\bar{\eta} \varphi}
\end{aligned}
$$

which is the bottom line for deriving the WT identities using a general unitary transformation.

\section{1-action}

We denote 42 in the following way (recalling that $[\alpha]$ is a triple index, while $\mu, \nu$ are single indices, and a dot notifies the position of remaning indices which, below, are integrated)

$$
\begin{aligned}
& 0=\frac{1}{Z(\eta, \bar{\eta})} \int d \mu_{C}(\bar{\varphi}, \varphi)\left(-\bar{\varphi}_{\mu .}^{0,2} \eta_{\nu .}^{0,2}+\bar{\eta}_{\mu .}^{0,2} \varphi_{\nu .}^{0,2}-\bar{\varphi}_{. \mu}^{1,3} \eta_{. \nu}^{1,3}+\bar{\eta}_{. \mu}^{1,3} \varphi_{. \nu}^{1,3}\right. \\
& \left.+\delta_{\varphi_{\nu .}^{0,2}} C_{\mu .[h]}^{0,2} \delta_{\bar{\varphi}_{[h]}^{0,2}}-\delta_{\varphi_{[h]}^{0,2}} C_{[h] \nu .}^{0,2} \delta_{\bar{\varphi}_{\mu .}^{0,2}}+\delta_{\varphi_{. \nu}^{1,3}} C_{. \mu[h]}^{1,3} \delta_{\bar{\varphi}_{[h]}^{1,3}}-\delta_{\varphi_{[h]}^{1,3}} C_{[h] . \nu}^{1,3} \delta_{\bar{\varphi}_{. \mu}^{1,3}}\right) e^{-S^{\mathrm{int}}(\varphi, \bar{\varphi})+\bar{\varphi} \eta+\bar{\eta} \varphi}
\end{aligned}
$$

Using $C_{[a][b]}^{i j}=\bar{C}_{[b][a]}^{i j}$, the above computes to

$$
\begin{aligned}
& 0=\frac{1}{Z(\eta, \bar{\eta})} \int d \mu_{C}(\bar{\varphi}, \varphi)\left(-\bar{\varphi}_{\mu .}^{0,2} \eta_{\nu .}^{0,2}+\bar{\eta}_{\mu .}^{0,2} \varphi_{\nu .}^{0,2}-\bar{\varphi}_{. \mu}^{1,3} \eta_{. \nu}^{1,3}+\bar{\eta}_{. \mu}^{1,3} \varphi_{. \nu}^{1,3}\right. \\
& \left.+\delta_{\varphi_{\nu .}^{0,2}} C_{\mu \cdot[h]}^{0,2} \delta_{\bar{\varphi}_{[h]}^{0,2}}-\delta_{\bar{\varphi}_{\mu .}^{0,2}} \bar{C}_{\nu \cdot[h]}^{0,2} \delta_{\varphi_{[h]}^{0,2}}+\delta_{\varphi_{. \nu}^{1,3}} C_{. \mu[h]}^{1,3} \delta_{\bar{\varphi}_{[h]}^{1,3}}-\delta_{\bar{\varphi}_{. \mu}^{1,3}} \bar{C}_{. \nu[h]}^{1,3} \delta_{\varphi_{[h]}^{1,3}}\right) e^{-S^{\text {int }}(\varphi, \bar{\varphi})+\bar{\varphi} \eta+\bar{\eta} \varphi} \\
& 0=\frac{1}{Z(\eta, \bar{\eta})} \int d \mu_{C}(\bar{\varphi}, \varphi)\left(-\bar{\varphi}_{\mu .}^{0,2} \eta_{\nu .}^{0,2}+\bar{\eta}_{\mu .}^{0,2} \varphi_{\nu .}^{0,2}-\bar{\varphi}_{. \mu}^{1,3} \eta_{. \nu}^{1,3}+\bar{\eta}_{. \mu}^{1,3} \varphi_{. \nu}^{1,3}\right. \\
& \left.+\delta_{\varphi_{\nu .}^{0,2}}\left[C \delta_{\bar{\varphi}}\right]_{\mu .}^{0,2}-\delta_{\bar{\varphi}_{\mu .}^{0,2}}\left[\delta_{\varphi} C^{\dagger}\right]_{\nu .}^{0,2}+\delta_{\varphi_{., 2}^{1,3}}\left[C \delta_{\bar{\varphi}}\right]_{. \mu}^{1,3}-\delta_{\bar{\varphi}_{. \mu}^{1,3}}\left[\delta_{\varphi} C^{\dagger}\right]_{. \nu}^{1,3}\right) e^{-S^{\mathrm{int}}(\varphi, \bar{\varphi})+\bar{\varphi} \eta+\bar{\eta} \varphi} .
\end{aligned}
$$

Let us evaluate by Lemma 2 the functional derivative terms that we can write

$$
\begin{aligned}
& \frac{1}{Z(\eta, \bar{\eta})} \int d \mu_{C}(\bar{\varphi}, \varphi)\left(\left[C \delta_{\bar{\varphi}}^{0,2}\right]_{\mu .}^{0} \delta_{\varphi_{\nu .}^{0,2}}-\left[\delta_{\varphi} C^{\dagger}\right]_{\nu .}^{0,2} \delta_{\bar{\varphi}_{\mu .}^{0,2}}+\left[C \delta_{\bar{\varphi}}\right]_{. \mu}^{1,3} \delta_{\varphi_{. \nu}^{1,3}}-\left[\delta_{\varphi} C^{\dagger}\right]_{. \nu}^{1,3} \delta_{\bar{\varphi}_{. \mu}^{1,3}}\right) e^{-S^{\mathrm{int}}(\varphi, \bar{\varphi})+\bar{\varphi} \eta+\bar{\eta} \varphi} \\
& =\frac{1}{Z(\eta, \bar{\eta})} \int d \mu_{C}(\bar{\varphi}, \varphi)\left(\varphi_{\mu .}^{0,2} \delta_{\varphi_{\nu .}^{0,2}}-\bar{\varphi}_{\nu .}^{0,2} \delta_{\bar{\varphi}_{\mu .}^{0,2}}+\varphi_{. \mu}^{1,3} \delta_{\varphi_{. \nu}^{1,3}}-\bar{\varphi}_{. \nu}^{1,3} \delta_{\bar{\varphi}_{. \mu}^{1,3}}\right) e^{-S^{\mathrm{int}}(\varphi, \bar{\varphi})+\bar{\varphi} \eta+\bar{\eta} \varphi} \\
& =\frac{1}{Z(\eta, \bar{\eta})} \int d \mu_{C}(\bar{\varphi}, \varphi)\left\{\int_{h_{i j} h^{i j}}\right. \\
& \varphi_{\mu .}^{0}\left[\frac{-\lambda}{\sqrt{\delta^{N}(e)}} \bar{\varphi}_{. h_{13} h_{12}}^{1} \varphi_{h_{21} . h_{23}}^{2} \bar{\varphi}_{h_{32} h_{31} \nu}^{3}+\bar{\eta}_{\nu .}^{0}\right]+\varphi_{\mu}^{2}\left[\frac{-\lambda}{\sqrt{\delta^{N}(e)}} \varphi_{h_{03} \cdot h_{01}}^{0} \bar{\varphi}_{h_{10} h_{13} \nu}^{1} \bar{\varphi}_{. h_{31} h_{30}}^{3}+\bar{\eta}_{\nu .}^{2}\right] \\
& +\varphi_{. \mu}^{1}\left[\frac{-\bar{\lambda}}{\sqrt{\delta^{N}(e)}} \bar{\varphi}_{h^{03} h^{02} .}^{0} \bar{\varphi}_{\nu h^{20} h^{23}}^{2} \varphi_{h^{32} . h^{30}}^{3}+\bar{\eta}_{. \nu}^{1}\right]+\varphi_{. \mu}^{3}\left[\frac{-\bar{\lambda}}{\sqrt{\delta^{N}(e)}} \bar{\varphi}_{\nu h^{02} h^{01}}^{0} \varphi_{h^{10} . h^{12}}^{1} \bar{\varphi}_{h^{21} h^{20}}^{2}+\bar{\eta}_{. \nu}^{3}\right] \\
& -\bar{\varphi}_{\nu .}^{0}\left[\frac{-\lambda}{\sqrt{\delta^{N}(e)}} \varphi_{. h^{13} h^{12}}^{1} \bar{\varphi}_{h^{21} . h^{23}}^{2} \varphi_{h^{32} h^{31} \mu}^{3}+\eta_{\mu .}^{0}\right]-\bar{\varphi}_{\nu .}^{2}\left[\frac{-\lambda}{\sqrt{\delta^{N}(e)}} \bar{\varphi}_{h^{03} . h^{01}}^{0} \varphi_{h^{10} h^{13} \mu}^{1} \varphi_{. h^{31} h^{30}}^{3}+\eta_{\mu}^{2} .\right] \\
& -\bar{\varphi}_{. \nu}^{1}\left[\frac{-\bar{\lambda}}{\sqrt{\delta^{N}(e)}} \varphi_{h_{03} h_{02} .}^{0} \varphi_{\mu h_{20} h_{23}}^{2} \bar{\varphi}_{h_{32} . h_{30}}^{3}+\eta_{. \mu}^{1}\right]-\bar{\varphi}_{. \nu}^{3}\left[\frac{-\bar{\lambda}}{\sqrt{\delta^{N}(e)}} \varphi_{\mu h_{02} h_{01}}^{0} \bar{\varphi}_{h_{10} \cdot h_{12}}^{1} \varphi_{h_{21} h_{20} .}^{2}+\eta_{. \mu}^{3}\right]
\end{aligned}
$$




$$
\} e^{-S^{\mathrm{int}}(\varphi, \bar{\varphi})+\bar{\varphi} \eta+\bar{\eta} \varphi}
$$

that will be shortly denoted by

$$
\begin{aligned}
& \frac{1}{Z(\eta, \bar{\eta})} \int d \mu_{C}(\bar{\varphi}, \varphi)\left(\varphi_{\mu .}^{0,2} \bar{\eta}_{\nu .}^{0,2}-\lambda \varphi_{\mu .}^{0,2} \cdot\left[\bar{\varphi}^{1} \varphi^{2,0} \bar{\varphi}^{3}\right]_{\nu .}+\varphi_{. \mu}^{1,3} \bar{\eta}_{. \nu}^{1,3}-\bar{\lambda} \varphi_{. \mu}^{1,3} \cdot\left[\bar{\varphi}^{0} \bar{\varphi}^{2} \varphi^{3,1}\right]_{. \nu}\right. \\
& \left.-\bar{\varphi}_{\nu .}^{0,2} \eta_{\mu .}^{0,2}+\lambda \bar{\varphi}_{\nu .}^{0,2} \cdot\left[\varphi^{1} \bar{\varphi}^{2,0} \varphi^{3}\right]_{\mu .}-\bar{\varphi}_{. \nu}^{1,3} \eta_{. \mu}^{1,3}+\bar{\lambda} \bar{\varphi}_{. \nu}^{1,3} \cdot\left[\varphi^{0} \varphi^{2} \bar{\varphi}^{3,1}\right]_{. \mu}\right) e^{-S^{\mathrm{int}}(\varphi, \bar{\varphi})+\bar{\varphi} \eta+\bar{\eta} \varphi} .
\end{aligned}
$$

Inserting this last relation into $\mathrm{D} .12$, we get

$$
\begin{aligned}
0= & \frac{1}{Z(\eta, \bar{\eta})} \int d \mu_{C}(\bar{\varphi}, \varphi)\left[-\bar{\varphi}_{\mu .}^{0,2} \eta_{\nu .}^{0,2}+\bar{\eta}_{\mu .}^{0,2} \varphi_{\nu .}^{0,2}-\bar{\varphi}_{. \mu}^{1,3} \eta_{. \nu}^{1,3}+\bar{\eta}_{. \mu}^{1,3} \varphi_{. \nu}^{1,3}+\varphi_{\mu .}^{0,2} \bar{\eta}_{\nu .}^{0,2}-\bar{\varphi}_{\nu .}^{0,2} \eta_{\mu .}^{0,2}+\varphi_{. \mu}^{1,3} \bar{\eta}_{. \nu}^{1,3}-\bar{\varphi}_{. \nu}^{1,3} \eta_{. \mu}^{1,3}\right. \\
& \left.-\lambda \varphi_{\mu .}^{0,2} \cdot\left[\bar{\varphi}^{1} \varphi^{2,0} \bar{\varphi}^{3}\right]_{\nu .}-\bar{\lambda} \varphi_{. \mu}^{1,3} \cdot\left[\bar{\varphi}^{0} \bar{\varphi}^{2} \varphi^{3,1}\right]_{. \nu}+\lambda \bar{\varphi}_{\nu .}^{0,2} \cdot\left[\varphi^{1} \bar{\varphi}^{2,0} \varphi^{3}\right]_{\mu .}+\bar{\lambda} \bar{\varphi}_{. \nu}^{1,3} \cdot\left[\varphi^{0} \varphi^{2} \bar{\varphi}^{3,1}\right]_{. \mu}\right] e^{-S^{\mathrm{int}}(\varphi, \bar{\varphi})+\bar{\varphi} \eta+\bar{\eta} \varphi}
\end{aligned}
$$

where repeated colors are summed as well as arguments in dot. Equation (D.15) is the starting equation for deriving the WT identities in the case of unitary 1-action.

[1] D. V. Boulatov, "A Model of three-dimensional lattice gravity," Mod. Phys. Lett. A 7, 1629 (1992) arXiv:hep-th/9202074.

[2] H. Ooguri, "Topological lattice models in four-dimensions," Mod. Phys. Lett. A 7, 2799 (1992) arXiv:hep-th/9205090.

[3] L. Freidel, "Group field theory: An overview," Int. J. Theor. Phys. 44, 1769 (2005) arXiv:hep-th/0505016.

[4] D. Oriti, "The group field theory approach to quantum gravity," arXiv:gr-qc/0607032

[5] D. Oriti, "The group field theory approach to quantum gravity: some recent results," arXiv:0912.2441 [hep-th]].

[6] D. Oriti (ed.), "Approaches to quantum gravity: Towards a new understanding of space, time and matter," Cambridge Univ. Press., Cambridge (2009).

[7] S. Gielen and D. Oriti, "Discrete and continuum third quantization of Gravity," arXiv:1102.2226 [gr-qc].

[8] R. Gurau, "Colored Group Field Theory," Commun. Math. Phys. 304, 69 (2011), arXiv:0907.2582 [hep-th]].

[9] R. Gurau, "Topological Graph Polynomials in Colored Group Field Theory," Annales Henri Poincare 11, 565 (2010) arXiv:0911.1945 [hep-th]].

[10] R. Gurau, "Lost in Translation: Topological Singularities in Group Field Theory," Class. Quant. Grav. 27, 235023 (2010) arXiv:1006.0714 [hep-th].

[11] R. Gurau, "The 1/N expansion of colored tensor models," Annales Henri Poincare 12, 829 (2011) [arXiv:1011.2726 [gr-qc]].

[12] R. Gurau and V. Rivasseau, "The 1/N expansion of colored tensor models in arbitrary dimension," Europhys. Lett. 95, 50004 (2011) arXiv:1101.4182 [gr-qc]].

[13] R. Gurau, "The complete 1/N expansion of colored tensor models in arbitrary dimension," arXiv:1102.5759 [gr-qc].

[14] V. Bonzom, R. Gurau, A. Riello and V. Rivasseau, "Critical behavior of colored tensor models in the large N limit," Nucl. Phys. B 853, 174 (2011) arXiv:1105.3122 [hep-th]].

[15] R. Gurau, "A generalization of the Virasoro algebra to arbitrary dimensions," arXiv:1105.6072 [hep-th].

[16] F. Girelli and E. R. Livine, "A Deformed Poincare Invariance for Group Field Theories," Class. Quant. Grav. 27, 245018 (2010) arXiv:1001.2919 [gr-qc]].

[17] A. Baratin and D. Oriti, "Group field theory with non-commutative metric variables," Phys. Rev. Lett. 105, 221302 (2010) arXiv:1002.4723 [hep-th]].

[18] A. Baratin, F. Girelli and D. Oriti, "Diffeomorphisms in group field theories," Phys. Rev. D 83, 104051 (2011) arXiv:1101.0590 [hep-th]].

[19] S. Carrozza and D. Oriti, "Bounding bubbles: the vertex representation of 3d Group Field Theory and the suppression of pseudo-manifolds," arXiv:1104.5158 [hep-th].

[20] J. Ben Geloun, "Classical Group Field Theory," arXiv:1107.3122 [hep-th].

[21] J. Ben Geloun and V. Bonzom, "Radiative corrections in the Boulatov-Ooguri tensor model: The 2-point function," Int. J. Theor. Phys. 50, 2819 (2011) arXiv:1101.4294 [hep-th]].

[22] V. Rivasseau, "Towards Renormalizing Group Field Theory," PoS C NCFG2010, 004 (2010) arXiv:1103.1900 [gr-qc]].

[23] L. Freidel, R. Gurau and D. Oriti, "Group field theory renormalization - the 3d case: power counting of divergences," Phys. Rev. D 80, 044007 (2009) arXiv:0905.3772 [hep-th]].

[24] J. Magnen, K. Noui, V. Rivasseau and M. Smerlak, "Scaling behavior of three-dimensional group field theory," Class. Quant. Grav. 26, 185012 (2009) arXiv:0906.5477][hep-th]].

[25] J. Ben Geloun, J. Magnen and V. Rivasseau, "Bosonic Colored Group Field Theory," Eur. Phys. J. C 70, 1119 (2010) arXiv:0911.1719 [hep-th]].

[26] J. Ben Geloun, T. Krajewski, J. Magnen and V. Rivasseau, "Linearized Group Field Theory and Power Counting Theorems," Class. Quant. Grav. 27, 155012 (2010) arXiv:1002.3592 [hep-th]]. 
[27] V. Bonzom and M. Smerlak, "Bubble divergences from cellular cohomology," Lett. Math. Phys. 93, 295 (2010) arXiv:1004.5196 [gr-qc]].

V. Bonzom and M. Smerlak, "Bubble divergences: sorting out topology from cell structure," arXiv:1103.3961 [gr-qc].

[28] J. Ben Geloun, R. Gurau and V. Rivasseau, "EPRL/FK Group Field Theory," Europhys. Lett. 92, 60008 (2010) arXiv:1008.0354 [hep-th]].

[29] H. Grosse and R. Wulkenhaar, "Renormalization of phi**4 theory on noncommutative $\mathrm{R}^{* *} 4$ in the matrix base," Commun. Math. Phys. 256, 305 (2005) arXiv:hep-th/0401128.

[30] M. Disertori, R. Gurau, J. Magnen and V. Rivasseau, "Vanishing of Beta Function of Non Commutative Phi**4(4) Theory to all orders," Phys. Lett. B 649, 95 (2007) arXiv:hep-th/0612251.

[31] J. Ben Geloun, R. Gurau and V. Rivasseau, "Vanishing beta function for Grosse-Wulkenhaar model in a magnetic field," Phys. Lett. B 671, 284 (2009) arXiv:0805.4362 [hep-th]].

[32] J. Ben Geloun, in preparation.

[33] H. Grosse and R. Wulkenhaar, "Progress in solving a noncommutative quantum field theory in four dimensions," arXiv:0909.1389 [hep-th]. 